\title{
Performance Enhancement of Organic Light-Emitting Diodes with an Inorganically Doped Hole Transport Layer
}

Xiaomeng Li

Follow this and additional works at: https://researchrepository.wvu.edu/etd

\section{Recommended Citation}

$\mathrm{Li}$, Xiaomeng, "Performance Enhancement of Organic Light-Emitting Diodes with an Inorganically Doped Hole Transport Layer" (2016). Graduate Theses, Dissertations, and Problem Reports. 6077.

https://researchrepository.wvu.edu/etd/6077

This Dissertation is protected by copyright and/or related rights. It has been brought to you by the The Research Repository @ WVU with permission from the rights-holder(s). You are free to use this Dissertation in any way that is permitted by the copyright and related rights legislation that applies to your use. For other uses you must obtain permission from the rights-holder(s) directly, unless additional rights are indicated by a Creative Commons license in the record and/ or on the work itself. This Dissertation has been accepted for inclusion in WVU Graduate Theses, Dissertations, and Problem Reports collection by an authorized administrator of The Research Repository @ WVU.

For more information, please contact researchrepository@mail.wvu.edu. 


\title{
Performance Enhancement of Organic Light-Emitting Diodes with an Inorganically Doped Hole Transport Layer
}

\author{
Xiaomeng Li
}

Dissertation Proposal submitted to the Benjamin M. Statler College of Engineering and

Mineral Resources at West Virginia University in partial fulfillment of the requirements

for the degree of

Doctor of Philosophy

In

Electrical Engineering

\author{
Xian-An Cao, Ph.D., Committee Chairperson \\ Parviz Famouri, Ph.D. \\ Mark Jerabek, Ph.D. \\ Lianshin Lin, Ph.D. \\ Yuxin Liu, Ph.D.
}

Lane Department of Computer Science and Electrical Engineering

Morgantown, West Virginia 2016

Keywords: Electronic Doping, Evaporation Deposition, Organic Light-Emitting Diode, Reliability, Transition Metal Oxide,

Copyright 2016 Xiaomeng Li 


\section{Abstract \\ Performance Enhancement of Organic Light-Emitting Diodes with an Inorganically Doped Hole Transport Layer \\ Xiaomeng Li}

Organic light-emitting diodes (OLEDs) are generally considered as the next generation display and lighting sources owing to their many attractive properties, including low power consumption, wide viewing angle, vibrant color, high contrast ratios and compatibility with flexible substrates. The research and development of OLEDs has attracted considerable interest and has led to significant progress during the last two decades. The use of OLEDs in small-area displays such as cell phone screens, digital cameras, and wearable devices has become a reality. However, the OLED technology is still far from mature, posing a challenge for their widespread acceptance for applications in large-area displays and solid-state lighting. In particular, the lifetime of OLEDs is too short for many commercial applications, and the degradation mechanisms are still under debate. This work aims to improve the OLED device lifetime by doping of organic hole transport materials with inorganic transition metal oxides (TMOs), and to reduce the cost by simplifying the device layer structure and manufacturing procedure.

First, stress tests under continuous wave and pulsed currents were conducted to gain a better understanding of the key factors governing the degradation process of phosphorescent OLEDs. Through comparative studies of the aging behaviors of OLEDs with different hole transport layers (HTLs) under different stressing conditions, we have found that joule heating plays an important role in device degradation when a large energy level misalignment exists at the indium-tin-oxide (ITO) anode/HTL interface. The heating was effectively suppressed by reducing the interfacial energy barrier, leading to a prolonged lifetime of the OLEDs.

P-type doping of hole transport materials with TMOs was then developed as an effective way to reduce the interfacial energy barrier and the operational voltage of OLED devices. A systematical study was carried out on the effects of doping 4,4'-Bis(N-carbazolyl)-1,1'-biphenyl (CBP), a wide bandgap organic hole transport material, with $\mathrm{WO}_{3}$ and $\mathrm{MoO}_{3}$. The optimal doping conditions including the doping 
level and doping thickness have been determined by fabricating and characterizing a series of hole-only devices. Integrating the doped HTL into green phosphorescent OLEDs has resulted in a simplified structure, better optoelectronic characteristics, and improved device reliability.

Finally, selective doping of organic materials with the TMOs was developed and the concept of delta doping was applied to OLEDs for the first time. Selective doping was achieved by simple sequential deposition of the organic host and TMO dopant. Hole-only devices with a HTL comprising alternative 0.5 nm TMO-doped/3-10 nm undoped CBP layers exhibited greatly enhanced hole transport and had a turnon voltage as low as $1.1 \mathrm{~V}$. Simple fluorescent tris-(8-hydroxyquinoline) aluminum (Alq 3 )-based green OLEDs with a selectively doped CBP HTL showed a lower voltage and longer lifetime under constantcurrent stressing compared to similar OLEDs with an undoped HTL. Furthermore. delta doping was realized in more thermally stable organic materials, resulting in a marked conductivity increase along the plane of the doped layers by several orders of magnitude. The delta doping effects were explained by hole accumulation in potential wells formed in nanometer-thick doped regions, as revealed by high-resolution secondary ion mass spectrometry (SIMS) measurements. 


\section{Acknowledgments}

I would like to thank my parents for their constant love and support of all of my endeavors. Without them, I would not have made it this far.

I would especially like to thank my advisor Prof. Xian-An Cao for offering me the opportunity to work on a very interesting research topic of inorganic doping in organic lightemitting diodes, for providing excellent experimental facilities, for his kind support and guidance on my research work, and for his expertises, motivation and enthusiasm which have always inspired me to greater efforts.

I wish to thank Prof. Parviz Famouri, Prof. Mark Jerabek, Prof. Lianshin Lin and Prof. Yuxin Liu, for serving on my committee and for their valuable suggestions and comments on my research work.

In addition, I wish to thank Dr. Weiqiang Ding and Dr. Kolin Brown of WVU Shared Research Facilities for their patient training and timely technical support.

All my groupmates and colleagues have made the research lab a wonderful place to work. In particular, I would like to thank Renyuan Yang, David. Shelhammer, Rajeev Acharya, and Yifei Lu, for their friendship and help in the past 4 years.

Finally, and most importantly, I thank my wife and friend, Xiaoyu Liu, and my son, Isaac $\mathrm{Li}$, for giving me up to long hours and late nights in lab. They have been a constant source of strength. To them I dedicated this dissertation. 


\section{TABLE OF CONTENTS}

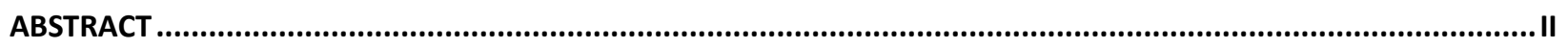

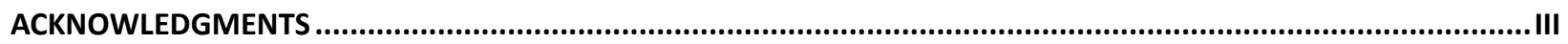

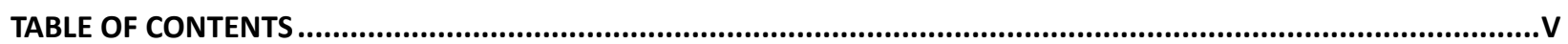

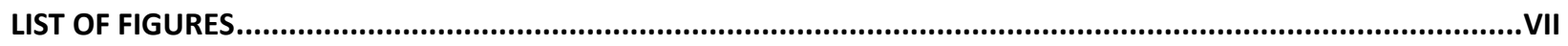

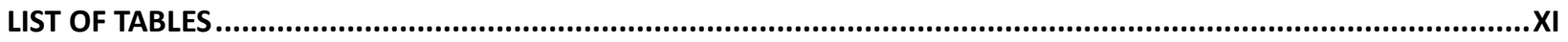

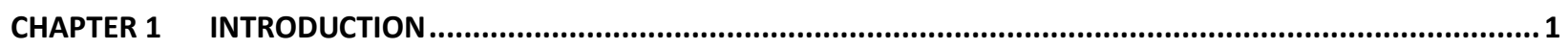

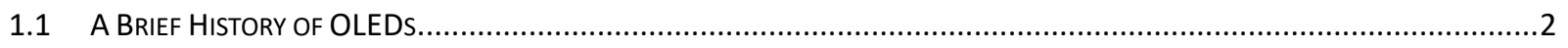

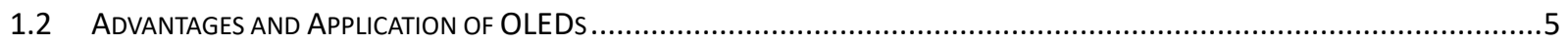

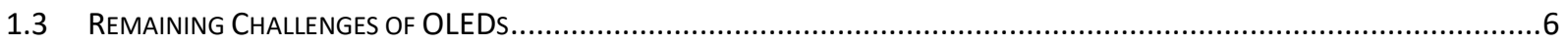

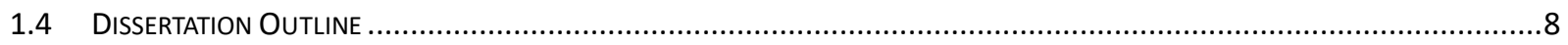

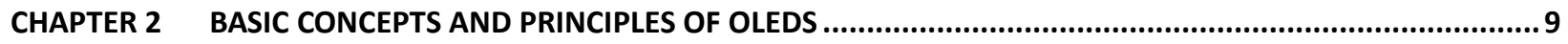

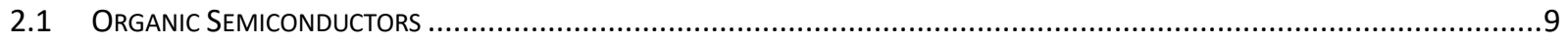

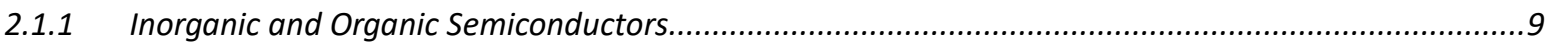

2.1.2 Energy Levels of Organic Semiconductors .......................................................................... 10

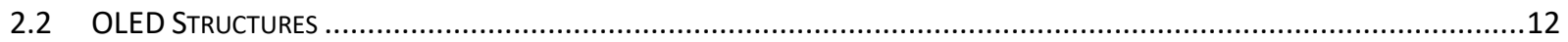

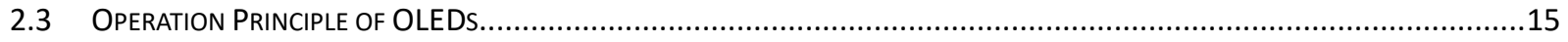

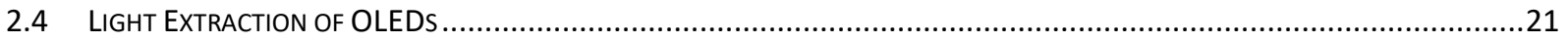

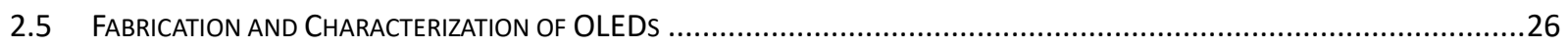

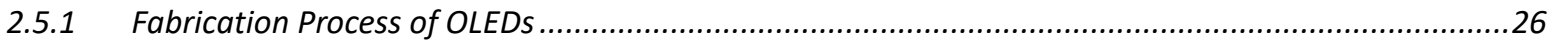

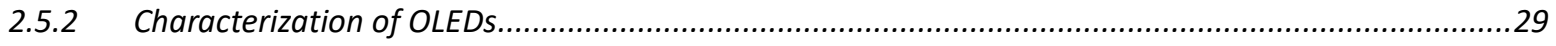

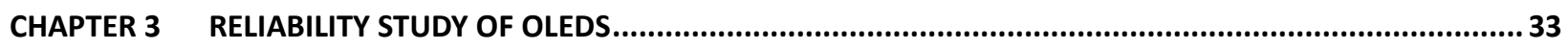

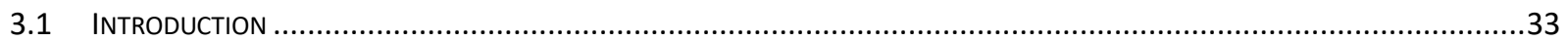




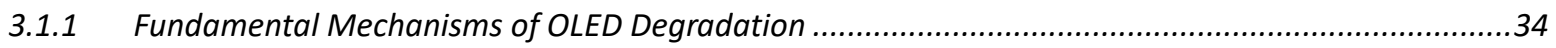

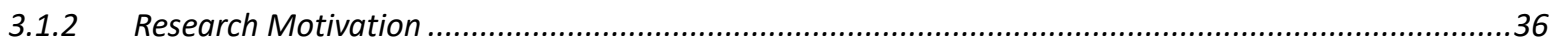

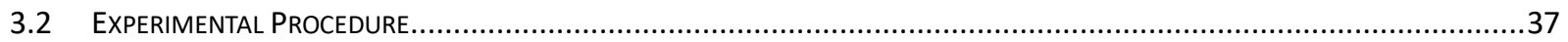

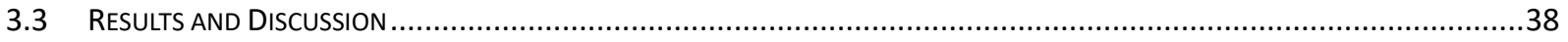

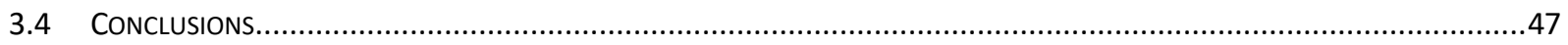

CHAPTER 4 P-TYPE DOPING OF HOLE TRANSPORT MATERIALS WITH INORGANIC TRANSITION METAL OXIDES

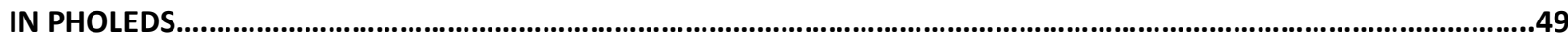

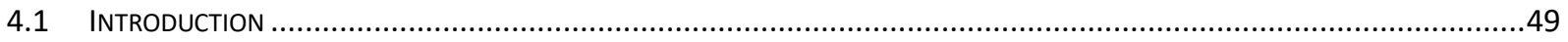

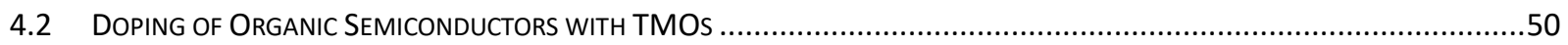

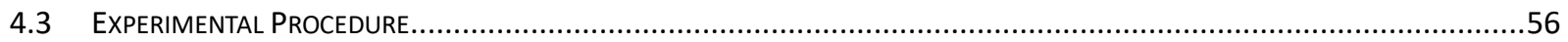

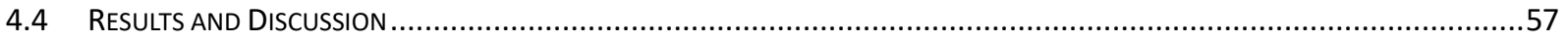

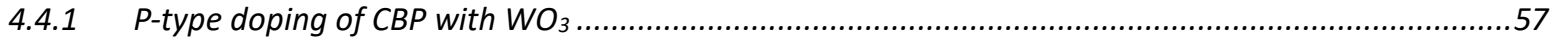

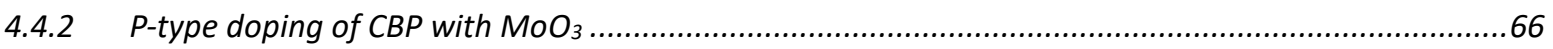

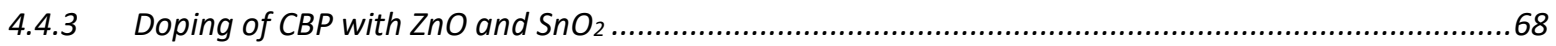

\section{CHAPTER 5 SELECTIVE DOPING OF ORGANIC HOLE TRANSPORT MATERIALS WITH INORGANIC TRANSITION}

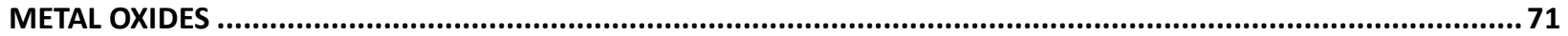

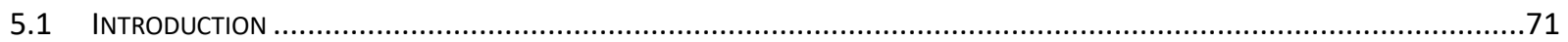

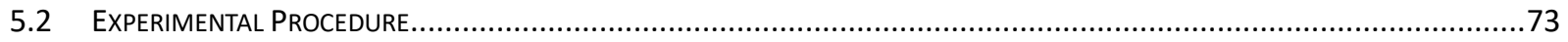

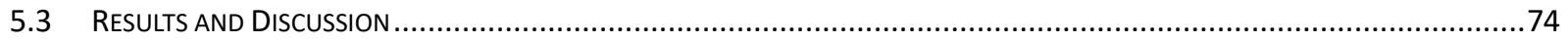

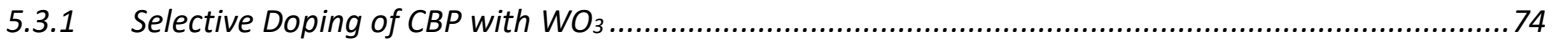

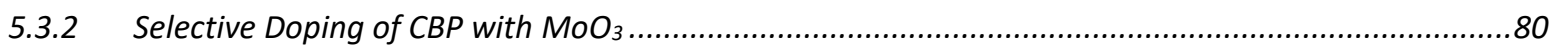

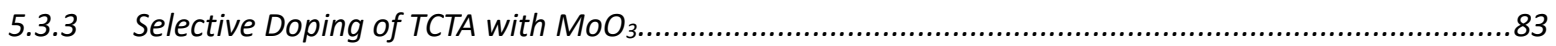

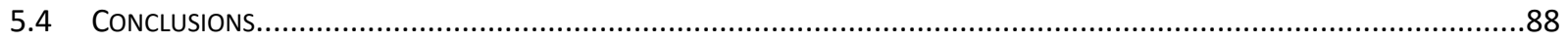

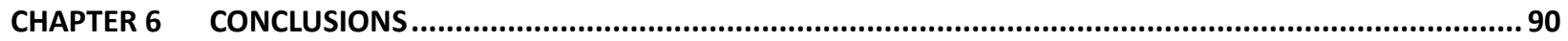

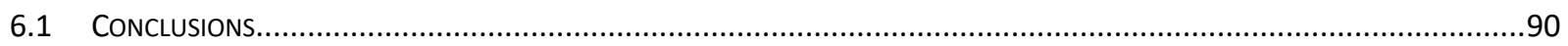

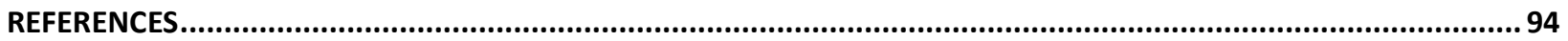




\section{LIST OF FIGURES}

Figure 1.1 Los Angeles, CA Citywide Streetlight Retrofit (2008-2015) [7] ............................. 1 Figure 1.2 (a) Complex OLED structure with several layers needed to aid carrier injection (b) Simplified OLED structure with ideal high work function anode ......................................... 7 Figure 2.1 Schematic energy band diagram of a semiconductor and effect of doping on energy levels

Figure 2.2 Hybridization of the valence shell electrons of a carbon atom. The upper and lower panel show $\mathrm{sp}^{3}$ and $\mathrm{sp}^{2}$ hybridization respectively [43].... 10 Figure 2.3 Energy level splitting of orbitals in a conjugated polymer according to molecular orbital theory (a) HOMO and LUMO level referring to the $\pi$ and $\pi^{*}$ bands (b) Collection of molecular orbitals forming bands separated by an energy gap [44] ..................................... 11

Figure 2.4 Basic multi-layered structure of OLEDs ..................................................... 13

Figure 2.5 Schematic diagram of OLED operation .................................................... 15 Figure 2.6 Jablonski diagram showing electron spin, absorption, fluorescence, phosphorescence and intersystem crossing. ........................................................................................ 20 Figure 2.7 Schematic of multi-layer OLED structure and optical ray diagram of light propagation via various modes, i.e., substrate escape, substrate wave-guided mode and ITO/ organic waveguided modes. 22

Figure 2.8 Schematic illustration of optical ray trajectories of light generated from OLED in a thin film having mesh structure on the backside of glass substrate [78] ................................. 23 Figure 2.9 Schematic representation of OLEDs with silica micro-spheres as scattering medium 24 Figure 2.10 Schematic ray optical diagram with ordered micro-lenses on the backside of glass substrate 25

Figure 2.11 Example of spin coating a small molecule in solution using a static dispense. The substrate is coated in the ink containing the molecules dissolved in a solvent (1). Then the substrate is rotated at high speed and the majority of the ink is flung off the side (2). Airflow then dries the majority of the solvent leaving a plasticised film (3) before the film fully dries to just leave the molecules on the surface (4) .......................................................................... 26 Figure 2.12 Travato Thermal Evaporation System connected to a $\mathrm{N}_{2}$ filled glovebox. .............. 28 Figure 2.13 A typical J-V curve of an OLED device .................................................... 29 Figure 2.14 Typical white OLED spectrum is generated by three different emitters (blue, green, and red). 30 Figure 3.1 Overview of relevant OLED brightness. Current lifetime data are from the state-ofthe-art red (red $\mathbf{\square}$ ) and white (O) OLEDs from Meerheim et al. and Loser et al. respectively [92]. 33

Figure 3.2 Intrinsic and extrinsic causes and mechanisms for OLED degradation [96] ............. 36 Figure 3.3 The schematic diagram of electric circuit. 37 
Figure 3.4 Layer structure of the green phosphorescent OLEDs and two sets of OLED devices are fabricated and tested with different types of HTL. 38

Figure 3.5 Frequency-dependent EL intensity of green phosphorescent OLEDs at different pulsed current densities. 39

Figure 3.6 (a)EL spectra of a phosphorescent OLED before and after stressing under $50 \mathrm{~mA} / \mathrm{cm}^{2}$ pulsed current $(1 \mathrm{kHz}, 20 \%$ duty cycle) for $60 \mathrm{~h}$. (b)The micrographs of the unstressed and stressed OLEDs operating at $20 \mathrm{~mA} / \mathrm{cm}^{2}$ 40 Figure 3.7 L-J-V characteristics of a phosphorescent OLED before (solid lines) and after (dashed

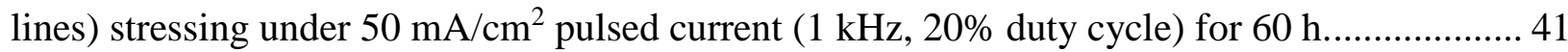
Figure 3.8 Forward J-V characteristics of phosphorescent OLEDs before and after stressing under $50 \mathrm{~mA} / \mathrm{cm}^{2} \mathrm{CW}$ current for $22 \mathrm{~h}$ or pulsed current $(1 \mathrm{kHz}, 10 \%$ duty cycle) for $89 \mathrm{~h} . \ldots . . .42$ Figure 3.9 Evolution of the normalized luminance of phosphorescent OLEDs stressed under 50 $\mathrm{mA} / \mathrm{cm}^{2} \mathrm{CW}$ or $1 \mathrm{kHz}$ pulsed currents. The pulse duty cycle varies from $10 \%$ to $50 \%$. 43 Figure 3.10 (a) Forward J-V characteristics of phosphorescent OLEDs before and after pulsed stressing at $50 \mathrm{~mA} / \mathrm{cm}^{2}(1 \mathrm{kHz}, 50 \%$ duty cycle) with and without a $-9 \mathrm{~V}$ bias. (b) Evolution of the normalized luminance of the phosphorescent OLEDs during $50 \mathrm{~mA} / \mathrm{cm}^{2}$ pulsed stressing

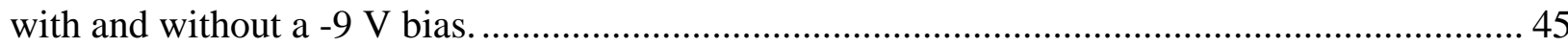

Figure 3.11 Band diagram of PhOLEDs with CBP HTL(left) and NPB HTL(right) ................... 46 Figure 3.12 Evolution of the normalized luminance of PhOLEDs with CBP HTL stressed CW

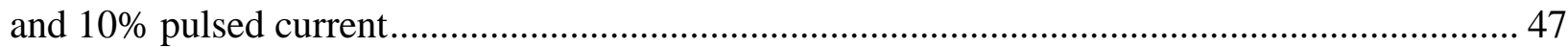

Figure 4.1 Charge transfer complex formation in p-type doping system .................................... 51

Figure 4.2 UPS and IPES spectra of vacuum grown $\mathrm{MoO}_{3}, \mathrm{~V}_{2} \mathrm{O}_{5}$ and $\mathrm{WO}_{3} \ldots \ldots \ldots \ldots \ldots \ldots \ldots \ldots \ldots . . . . . . . . . . . . . . .53$

Figure 4.3 Electrical Band Structure for some Transition Metal Oxide ...................................... 55 Figure 4.4 Schematic layer structure of (a) hole-only devices and (b) simplified PhOLED devices

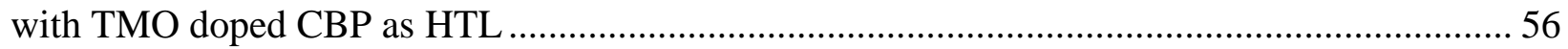
Figure 4.5 Energy diagram of hole-only device with a $\mathrm{WO}_{3}$-doped CBP HTL. ........................... 58 Figure 4.6 Absorption of thin film comprising $100 \mathrm{~nm} 30 \mathrm{~mol}_{\%} \mathrm{WO}_{3}$ doped CBP and $100 \mathrm{~nm}$ undoped $\mathrm{CBP}$ 58

Figure 4.7 J-V characteristics of hole-only devices with a $40 \mathrm{~nm} 10-40 \mathrm{~mol} \% \mathrm{WO}_{3}-\mathrm{doped} / 60 \mathrm{~nm}$

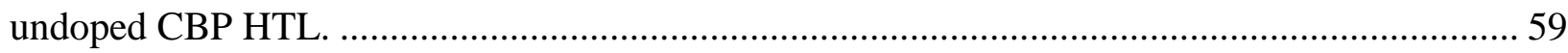

Figure 4.8 (a) J-V characteristics of hole-only devices with a $5-40 \mathrm{~nm} 30 \mathrm{~mol} \% \mathrm{WO}_{3}$-doped/60 $\mathrm{nm}$ undoped CBP HTL. (b) The device voltage at $100 \mathrm{~mA} / \mathrm{cm}^{2}$ as a function of the doped CBP layer thickness.

Figure $4.9 \mathrm{~J}-\mathrm{V}$ characteristics of hole-only devices with a HTL comprising $100 \mathrm{~nm}$ undoped CBP, $100 \mathrm{~nm} 30 \mathrm{~mol} \% \mathrm{WO}_{3}$-doped CBP, or $0.5 \mathrm{~nm} \mathrm{WO} \mathrm{W}_{3} / 100 \mathrm{~nm}$ undoped CBP....................... 61 Figure 4.10 (a)J-V characteristics of hole-only devices with a $100 \mathrm{~nm}$ doped CBP HTL or an $\mathrm{x} / 100-\mathrm{x}$ nm doped/undoped CBP HTL and (b) the band diagram of a doped/undoped CBP junction.

Figure $4.11 \mathrm{~J}-\mathrm{V}$ characteristics of green PhOLEDs with different CBP HTLs............................. 63 Figure 4.12 (a) L-J and (b) L-V characteristics of green PhOLEDs with different CBP HTLs ... 64 Figure 4.13 Evolution of the raw (a) and normalized (b) luminance of green PhOLEDs with 
different CBP HTLs under constant-current stressing at $50 \mathrm{~mA} / \mathrm{cm}^{2}$. 65

Figure $4.14 \mathrm{~J}-\mathrm{V}$ characteristics of hole-only devices with CBP doped with $10-40 \mathrm{~mol} \% \mathrm{MoO}_{3}$ as HTL compared to undoped CBP. 66

Figure $4.15 \mathrm{~J}-\mathrm{V}$ characteristics of hole-only devices with a HTL comprising $100 \mathrm{~nm} 30 \mathrm{~mol} \%$ $\mathrm{MoO}_{3}$ doped CBP, a HTL comprising $100 \mathrm{~nm} 30 \mathrm{~mol} \% \mathrm{WO}_{3}$ doped CBP, $0.5 \mathrm{~nm} \mathrm{MoO} / 100 \mathrm{~nm}$ undoped $\mathrm{CBP}$, or $0.5 \mathrm{~nm} \mathrm{WO}_{3} / 100 \mathrm{~nm}$ undoped CBP. 67

Figure 4.16 Schematic band diagram of the HOMO of CBP and the $\mathrm{CB}$ of $\mathrm{ZnO}$ and $\mathrm{SnO}_{2}$.

Electron transfer from the $\mathrm{CB}$ of $\mathrm{ZnO}$ and $\mathrm{SnO}_{2}$ to the $\mathrm{HOMO}$ of $\mathrm{CBP}$ results in a reduced hole concentration. 68

Figure 5.1 Schematic layer structures of hole-only devices with (a)uniformly doped HTL and (b) selectively doped HTL. Both hole-only devices have same $0.5 \mathrm{~nm} \mathrm{WO}_{3}$ as HIL. 73 Figure 5.2 Schematic layer structures of green $\mathrm{Alq}_{3}$ OLEDs with (a) uniformly doped HTL and (b) selectively doped HTL. Both OLED devices have a same $0.5 \mathrm{~nm} \mathrm{W_{3 }}$ HIL. 74 Figure 5.3 AFM scans of $100 \mathrm{~nm}$ CBP films over a $5 \mu \mathrm{m} \times 5 \mu \mathrm{m}$ area. Film (a) was undoped, film (b) was doped with $30 \mathrm{~mol}^{\%} \mathrm{WO}_{3}$ by co-deposition and film (c) was doped by sequential deposition of alternative $0.5 \mathrm{~nm} \mathrm{WO}_{3}$ and $3 \mathrm{~nm}$ CBP layers

Figure 5.4 (a)J-V characteristics of hole-only devices with a $100 \mathrm{~nm}$ CBP HTL comprising alternative $3 \mathrm{~nm}$ undoped spacer and $0.5 \mathrm{~nm}$ 40-100 $\mathrm{mol}_{\%} \mathrm{WO}_{3}$-doped CBP layers. (b)J-V data plotted on the log-log scale.

Figure 5.5 (a)J-V characteristics of hole-only devices with a $100 \mathrm{~nm}$ doped CBP HTL comprising alternative $3-10 \mathrm{~nm}$ undoped spacer and $0.5 \mathrm{~nm} \mathrm{WO} 3$ layers prepared by sequential deposition. (b) J-V characteristics of hole-only devices with a $100 \mathrm{~nm} 67 \mathrm{~mol} \% \mathrm{WO}_{3}$ doped CBP

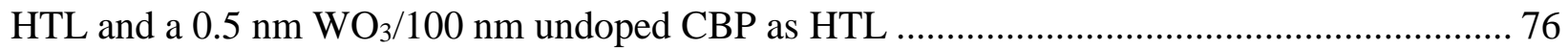
Figure 5.6 SIMS depth profile of tungsten in alternative $3 \mathrm{~nm} \mathrm{WO} / 15 \mathrm{~nm} \mathrm{CBP}$ thin films ....... 78 Figure 5.7 (a)J-V and (b)L-I characteristics of green OLEDs with an undoped CBP HTL or a CBP doped with $\mathrm{WO}_{3} \mathrm{HTL}$ by sequential deposition. 78

Figure 5.8 Transmission of thin films comprising $0.5 \mathrm{~nm} \mathrm{WO} / 60 \mathrm{~nm}$ undoped CBP or $60 \mathrm{~nm}$ sequential doped $\mathrm{CBP}$ with $\mathrm{WO}_{3}$ 79

Figure 5.9 Evolution of the normalized luminance of OLEDs with undoped and doped HTL stressed at a constant current density of $100 \mathrm{~mA} / \mathrm{cm}^{2}$ 80

Figure 5.10 Evolution of the normalized luminance of OLEDs with undoped and doped HTL stressed at a constant current density of $100 \mathrm{~mA} / \mathrm{cm}^{2}$.

Figure 5.11 (a) J-V and (b)L-J characteristic of OLEDs comprising undoped CBP as HTL and $\mathrm{MoO}_{3}$ selectively doped CBP as HTL

Figure 5.12 Evolution of the normalized luminance and operation voltage of OLEDs with an undoped or doped HTL stressed at a constant current density of $100 \mathrm{~mA} / \mathrm{cm}^{2}$. 83

Figure 5.13 SIMS depth profile of Mo in alternative $3 \mathrm{~nm} \mathrm{MoO} 3 / 15 \mathrm{~nm}$ TCTA thin films ........ 84 Figure $5.14 \mathrm{~J}-\mathrm{V}$ characteristic of hole-only devices with a (a)undoped/ $\mathrm{MoO}_{3}$ doped TCTA HTL

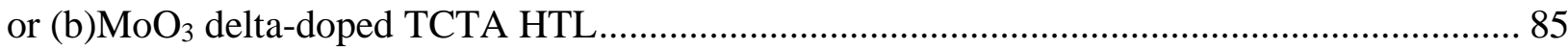
Figure 5.15 Schematic diagram of vertical and lateral current flows in delta-doped structures. . 87 Figure 5.16 Lateral J-V characteristic of hole-only devices with uniform doped TCTA and delta- 


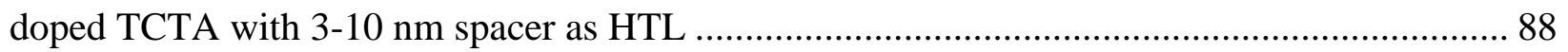




\section{LIST OF TABLES}

Table $3.1 \mathrm{t}_{0.8}$ and $\mathrm{t}_{0.5}$ of OLEDs stressed under $50 \mathrm{~mA} / \mathrm{cm}^{2} \mathrm{CW}$ current or $1 \mathrm{kHz}$ pulsed currents with different duty cycles................................................................................................. 44 Table 4.1 Energy levels of $\mathrm{MoO}_{3}, \mathrm{~V}_{2} \mathrm{O}_{5}$ and $\mathrm{WO}_{3}$ measured via UPS and IPES. Determined by XAS/XES measurements from band gap................................................................... 54

Table 5.1 Density, molar mass and molar volume of CBP, TCTA, $\mathrm{WO}_{3}$ and $\mathrm{MoO}_{3} \ldots \ldots \ldots \ldots . . . . . . . .72$

Table 5.2 Deposition rate ratio of $\mathrm{WO}_{3}$ vs. $\mathrm{CBP}$ and $\mathrm{MoO}_{3}$ vs. $\mathrm{CBP}$ under molar ratio from ..... 72 


\section{Chapter 1 Introduction}

In the 21 st century, many countries are increasingly aware of the urgent need to make better use of the world's energy resources. The recent energy crisis and global warming concerns have led to great interest in developing efficient device technologies for renewable energy generation and energy conservation. Among all the technologies under development is the solidstate lighting (SSL) based on light-emitting diodes (LEDs) and organic LEDs (OLEDs) [1-3]. SSL sources could be much more energy efficient than the conventional light sources such as incandescent and fluorescent lamps. Therefore, the development of SSL offers an opportunity to significantly lower worldwide energy consumption. About $21 \%$ of electricity is being used for the purpose of general illumination. If all conventional lighting sources are replaced by SSL sources with a luminous efficacy above $150 \mathrm{~lm} / \mathrm{W}$, more than $50 \%$ of the electricity currently used for lighting can be saved [4-6].
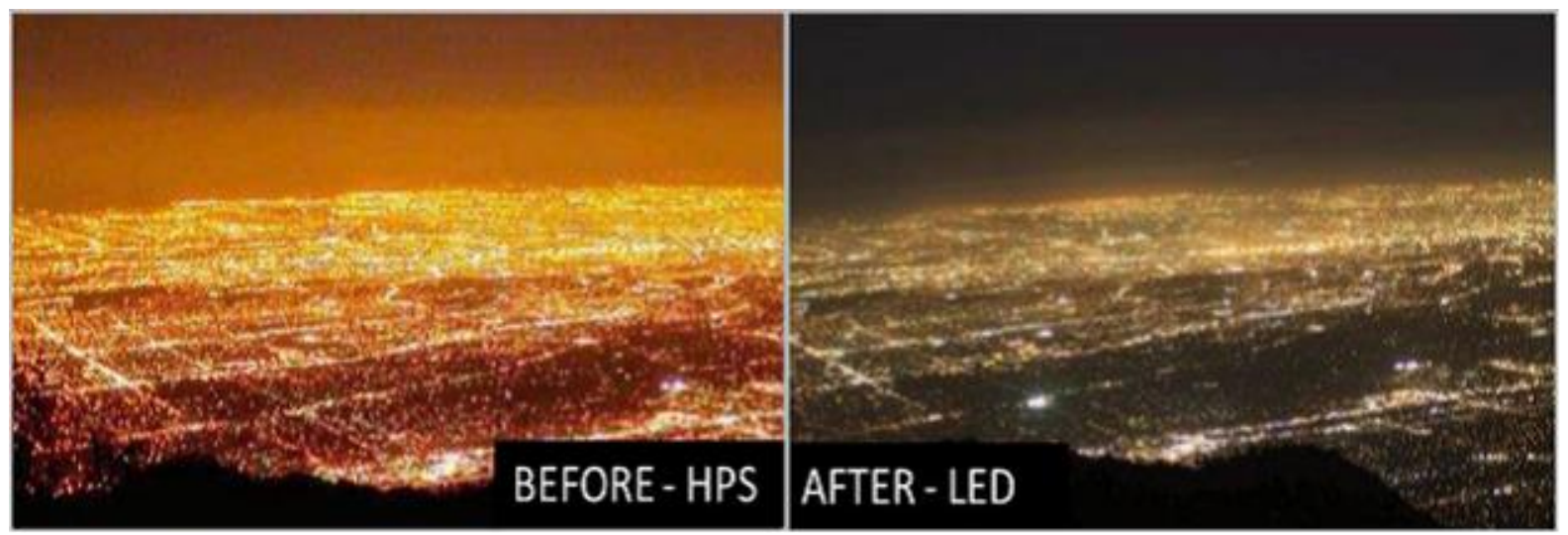

Figure 1.1 Los Angeles, CA Citywide Streetlight Retrofit (2008-2015) [7]

The SSL technology also enjoys the advantage of easy and efficient light management. In the past decade, new LED-based outdoor area lights have demonstrated the ability to provide suitable illuminance levels using significantly lower total light output than the conventional lighting products they have replaced. This is accomplished through an improved light distribution that reduces over-lighting of the target area, improves illuminance uniformity, and 
produces less wasted light falling outside the target area. Figure 1.1 compares the night landscapes of the city of Los Angeles before and after LED streetlite retrofits [7].

The OLED technology is generally viewed as a rising star in the field of SSL as an effective means to convert electrical power into light. OLEDs are also excellent lighting sources for flat-panel displays due to the fact that they can be built over a large area on many types of

substrates including flexible substrates. Many companies are currently trying to commercialize the OLED technology [8-11]. Yet, the technology still has lots of obstacles to overcome before the full potential can be realized. This dissertation focuses on studying the reliability issues of OLEDs and performance improvement of OLEDs by introducing inorganic dopants into organic charge transport materials. The results may have a significant technological impact on SSL and displays in the near future.

\subsection{A Brief History of OLEDs}

OLEDs has been developed and studied for almost 30 years having great potential to be the next generation lighting and flat panel display source. The OLED is a semiconductor $p-n$ junction device based on small-molecule or polymer organic materials capable of emitting light. When an external electric voltage is applied, light emission is generated due to the electroluminescence (EL) phenomenon.

The EL phenomenon was first discovered from an organic molecule (anthracene) was reported by Pope and coworkers in 1963 [12]. The EL was observed when a bias of several hundred volts was applied across a $10 \mu \mathrm{m}$ thick anthracene layer. In 1965 W. Helfrich and W.G. Schneider demonstrated double injection EL for the first time in an anthracene single crystal by injecting electrons and holes from separate electrodes [13]. In 1982, organic thin films were deposited in vacuum by Vincent et al. to achieve EL. Though the operation voltage was lowered below $100 \mathrm{~V}$, the external quantum efficiency (EQE) remained very low, below (0.05\%) [14]. In 1970s, EL was also observed from polymer films by R. Patridge at the National Physical 
Laboratory in the UK, and the first polymer light emitting diode (PLED) consisting of a film of poly(N-vinylcarbazole) (PVK) was reported. The results were patented in 1975 and published in 1983 [15]. However, due to relatively low conductivity of the polymer films, a very high driving voltage was required which limited the output power and efficiency of the device.

The first efficient OLED was developed by C.W. Tang and Van Slyke from Eastman Kodak in 1987 [16]. A low-voltage OLED was demonstrated with an emitting layer based on a small molecule organic material. The device has a bilayer organic thin film structure, which was prepared by thermal evaporated deposition. Holes and electrons were injected efficiently from an indium-tin-oxide (ITO) anode and an alloyed $\mathrm{Mg}$-Ag cathode. Electron-hole recombination and exciton generation were confined near the organic interface region. At a driving voltage below 10 $\mathrm{V}$, the external quantum efficiency of green EL was $1 \%$, the luminous efficiency reached 1.5 $\mathrm{lm} / \mathrm{W}$, and brightness was about $1000 \mathrm{~cd} / \mathrm{m}^{2}$.

In 1988, the first double heterostructure OLED was reported by Chihaya Adachi and Tetsuo Tsutsui, whose work led to the later commercialization of OLEDs [17]. Typical devices with the EL exceeding $1 \mu \mathrm{W} / \mathrm{cm}^{2}$ at the $2 \mathrm{~mA} / \mathrm{cm}^{2}$ injection current consisted of two electrodes, an electron transport layer (ETL), an emitting layer (EML) and a hole transport layer (HTL). This sandwiched structure paved the way for OLED devices with high efficiency and low operation voltage, commonly used nowadays.

In 1990 J. H. Burroughes et al. from Cavendish Lab. in Cambridge reported green polymer-based LEDs (PLEDs) with a driving voltage about $14 \mathrm{~V}$ with poly(p-phenylene vinylene) (PPV) [18]. The typical devices having ITO as the anode and Al as the cathode showed a quantum efficiency about $0.05 \%$ which was moderate at that time. The failure mode of these devices is usually associated with the accumulated joule heating at the heterointerfaces.

The first white OLED made from vacuum-deposited organic thin films was reported by J. Kido et al. in 1995 [19]. In their device, three emitter layers with different carrier transport properties, each emitting blue, green, or red light, were used to generate white light. Bright white 
light, over $2000 \mathrm{~cd} / \mathrm{m}^{2}$, nearly as bright as a fluorescent lamp, was successfully obtained at low drive voltages such as $15 \mathrm{~V}$ to $16 \mathrm{~V}$. White OLEDs can be used to construct paper-thin light sources, which are particularly useful for places that require lightweight illumination devices, such as in aircraft and space shuttles, or as sources for back-lighting in liquid crystal displays (LCDs) as well as full color displays, achieved by combining the emitters with micro-patterned color filters.

The EL of above-mentioned OLEDs and PLEDs arises from a fluorescent process, and the efficiency remained low despite significant efforts for improvement. In fluorescent dyes, only excitons in the singlet spin state induce fluorescent emission. The singlets represent a small fraction (about 25\%) of the total excited-state population (the remainder are triplet states).

Phosphorescent dyes offer a means of achieving improved light-emission efficiencies, as emission may result from both singlet and triplet states. High-efficiency phosphorescent OLEDs (PhOLEDs) with nearly $100 \%$ internal quantum efficiency (IQE) were developed by M.A. Baldo et al. in 1998 [20]. PhOLEDs emitting all colors have been developed by using emitting dyes based on iridium complexes.

In 2012, a white OLED was demonstrated by Panasonic with a high efficacy of $142 \mathrm{~lm} / \mathrm{W}$ at a luminance of $1,000 \mathrm{~cd} / \mathrm{m}^{2}$. There were many useful technological advances that led to this achievement, but the area of the device was only $4 \mathrm{~mm}^{2}$ and the technique used to enhance the extraction of light cannot be extended to large area at an acceptable cost while maintaining the slim profile of the panel. In 2013, an approach was used by NEC Lighting to make a $156 \mathrm{~lm} / \mathrm{W}$ device with the area of $2 \mathrm{~mm} \times 2 \mathrm{~mm}$ and luminance of $1000 \mathrm{~cd} / \mathrm{m}^{2}$. Incorporating the same technology into a panel resulted in an efficacy of $75 \mathrm{~lm} / \mathrm{W}$ [21-22].

To date, the OLED lighting technology has reached a stage where successful commercialization of products is feasible. The performance, in terms of efficacy, lifetime and color, is competitive with other energy efficient lighting technologies such as fluorescent lamps and LED luminaires. Luminaires with exciting new form factors are moving from concept 
studies to the market-place. One key obstacle to adoption of OLED lighting remains to be the cost, but substantial progress was reported in recent years. It is possible that near term adoption of OLED lighting products will generate sufficient interest and revenue to support the development of manufacturing technology to enable further cost reductions.

\subsection{Advantages and Application of OLEDs}

The OLED technology has several advantages compared to the traditional lighting and display technology [23-25]. The energy-saving potential of OLEDs is similar to that of LEDs, but the two technologies differ in a number of ways. For one thing, whereas LEDs are concentrated sources of bright light, OLEDs can be configured as larger-area, more diffuse light sources, which may be more practical for general ambient lighting because the soft light can be viewed directly, with less need for shades, diffusers, lenses, louvers, or parabolic shells. The diffuse light from OLEDs allows them to be used very close to the task surface without creating glare for the user, which means that less total light can be used in order to achieve desired illuminance levels.

OLEDs can be made very thin, increasing their eye appeal and allowing for easy attachment to the surfaces of walls and ceilings. This, coupled with the diffuse nature of OLED lighting, could enable entirely new types of light fixtures that are both attractive and highly efficient. OLEDs can also be made in almost any shape, can be deposited on flexible substrates, and can be transparent, emitting light from both sides of the device, features that greatly expand the design possibilities, allowing for a completely new lighting experience.

Compared to LCDs, today's dominant flat panel display (FPD) technology, OLEDs are capable of providing markedly better performance features. Thinner and lighter OLEDs offer much faster response times, wider viewing angles, higher contrast ratios and brighter, more saturated colors for a more enjoyable viewing experience. With operating lifetimes now in the tens of thousands of hours, OLED-based flat-panel displays can also be more energy efficient 
than LCD displays.

OLEDs also have the potential to be more cost effective. They have fewer processing steps and are also less materials and equipment intensive than today's LCDs. As the manufacturing technologies mature, OLED production yields should continue to improve and larger-scale equipment be brought on line. As a result, production costs will continue to decrease, ultimately enabling OLEDs to outperform LCDs on a cost basis.

\subsection{Remaining Challenges of OLEDs}

Over the past decade, OLEDs have undergone a phenomenal development effort and their performance meets many of the targets necessary for color displays. However, they have yet to deliver the desired efficiency and stability required for general illumination. Several challenges facing material and device development listed below still need to be conquered [26-29].

At First, the OLED lifetime is still much shorter compared to traditional display and LED technology. The half-life of OLED displays is about 14,000 hours as compared to $25,000-40,000$ hours for LCD, LED technologies. In the SSL area, the lifetime is 10,000-25,000 hours for white OLEDs which is significantly shorter than 40,000-50,000 hours for white LEDs [26]. Fast OLED degradation can be attributed to the poor stability of organic materials, caused by both extrinsic factors and intrinsic factors. The extrinsic factors including introduced oxygen, water as well as impurities generated during device fabrication process, are relatively well understood and easy to control. However, the intrinsic factors including charge migration and accumulation, exciton caused reaction and re-orientation of dipoles, are more difficult to investigate. In particular, reliable blue phosphorescent dyes are still not available. Blue PhOLEDs have a short lifetime which is only $30 \%$ of that of red and green PhOLEDs [30-31].

Secondly, OLEDs suffer from the relatively low charge mobility of organic materials as well as large energy barriers between the electrodes and organic materials, and thus generally have a large operating voltage and poor reliability. In a typical OLED structure, injection layers 
are added to the device to match the energy levels in a stepwise fashion, as shown in Figure 1.2(a) [32]. By doing this, charge injection is improved. However, these additional layers add to the complexity of the device structure and manufacturing process. They can be detrimental to the device performance by impeding the charge transport and causing interfacial reactions. Therefore, there is a need to improve charge transport in OLEDs, and to simplify the OLED structure by eliminating the injection layers without causing an increase in the interfacial energy barriers (Figure 1.2(b)) and the device operating voltage.

(a)

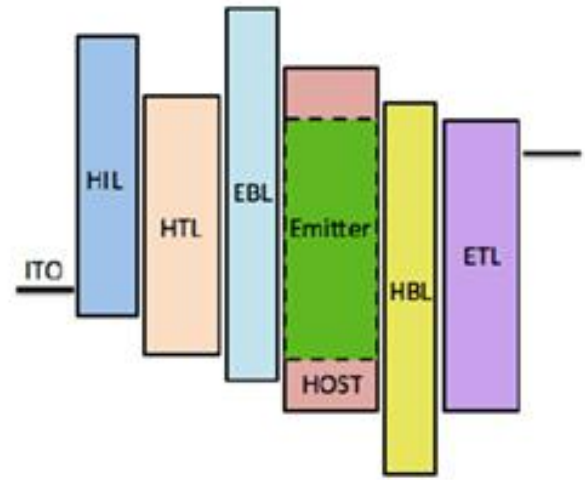

(b)
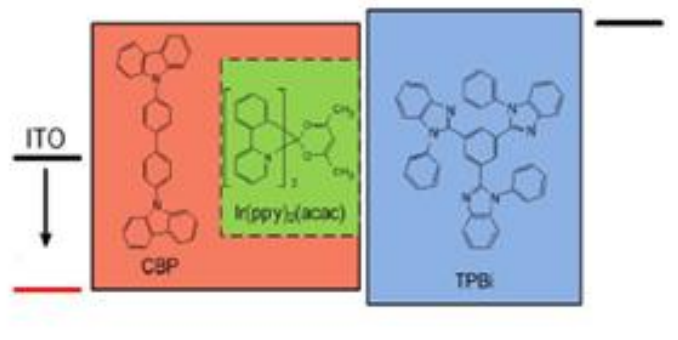

Figure 1.2 (a) Complex OLED structure with several layers needed to aid carrier injection (b) Simplified OLED structure with ideal high work function anode[32]

Thirdly, even though PhOLEDs can achieve a $~ 100 \%$ internal quantum efficiency (IQE), the external quantum efficiency (EQE) is only 20\% [33-35]. This is mainly due to poor light extraction from the OLED device, where a wave guide exists due to large contrasts in the refractive indices between the organic, electrode and substrate materials. When light is generated from thin organic emitting layers spontaneously in all directions, the light with incidence angles larger than critical angles will undergo the total internal reflection. In a planar OLED, 30\% light is trapped inside the glass substrate and $\sim 50 \%$ light is propagated through ITO-organic waveguided modes. Several light extraction technologies have been applied to improve the EQE of OLEDs including using rough surface of substrates and applying micro-cavity, improving the EQE remarkably to $40 \%$ 50\% [36-38]. However, there is still plenty of room for improvement 
and new efficient light extraction technologies should be further explored [39-40].

\subsection{Dissertation Outline}

As discussed above, it is clear that, in order to develop high performance and low cost OLED devices for efficient energy conversions, it is essential to investigate key material and structural factors causing OLED degradation and new techniques which can enable voltage reduction and structural simplification. This dissertation aims to improve the OLED performance and simplify the OLED structure by doping hole transport materials with transition metal oxides (TMOs). The research has four areas of focus, which are briefly described below.

Chapter 2 describes the OLED structure, basic working principle of OLEDs, theory of phosphorescent luminance, light extraction technology and fabrication method and characterizations of OLEDs.

Chapter 3 investigates the degradation mechanisms of PhOLEDs and presents a unique way to study the joule heating effect on the PhOLEDs aging behavior through pulsed current stressing which differentiates the thermal and nonthermal aging factors.

In Chapter 4, uniform p-type doping of wide bandgap CBP with $\mathrm{TMOs}, \mathrm{MoO}_{3}$ and $\mathrm{WO}_{3}$ is conducted and optimized. The effects of doping on the electrical characteristics, luminance efficiency, and reliability of PhOLED devices are studied. The effects of $\mathrm{ZnO}$ and $\mathrm{SnO}_{2}$ doping are also investigated in order to verify the p-type doping mechanism.

In Chapter 5, the concept of selective doping is applied to organic materials and OLEDs for the first time and a new delta doping technique is developed by simple sequential deposition. The effects of selective doping of CBP and TCTA with $\mathrm{WO}_{3}$ and $\mathrm{MoO}_{3}$ are investigated and compared to that of uniform doping. The above research work is summarized in Chapter 6 and future work is briefly discussed. 


\section{Chapter 2 Basic Concepts and Principles of OLEDs}

\subsection{Organic Semiconductors}

Organic semiconductors are such type materials, which are semiconducting in nature and based upon interactions between organic molecules. Carbon based organic materials have been used in electronics industry pertaining to its insulating property until the discovery of conducting polymers in 1976. The emergence of a new class of conducting organic materials, called $\pi$ conjugated organic materials, opened doors to their use in various optoelectronic devices.

\subsubsection{Inorganic and Organic Semiconductors}

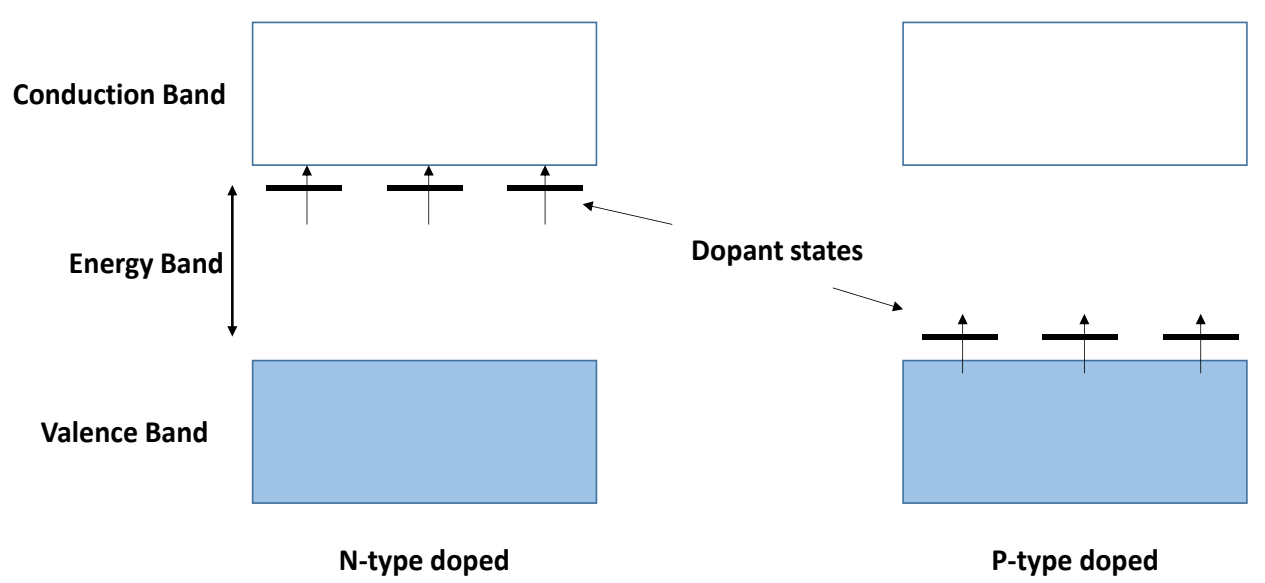

Figure 2.1 Schematic energy band diagram of a semiconductor and effect of doping on energy levels

According to the Bloch-Wilson theory of conduction [41], energy levels of a material form bands, which are filled with electrons from the material. A semiconductor material has a filled valence band (VB), and a relatively small energy band gap (Eg) that allows for thermal excitation of electrons from the filled valence band into the empty conduction band (CB). In addition, impurities can be intentionally added into the semiconductor material with energy 
levels inside the band gap to allow for excess carriers in the semiconducting material. This process is known as doping and a schematic of doping in a semiconductor is shown in Figure 2.1.

However, organic semiconductors are different from conventional crystalline semiconductors (such as $\mathrm{Si}$ and $\mathrm{GaN}$ ) in many ways. This leads to significant differences in their physical and electrical properties.

The interactions between organic semiconductors are based upon organic molecules as fundamental units and the interacting force is the weak Van der Waals force, as compared to the atomic units and covalent force for traditional crystalline semiconductors [42]. In addition, the small molecule organic semiconductor materials form amorphous thin films. Thus, the individual molecular units are isolated and lack a long-range order. As a result, the properties of organic semiconductors (i.e., dynamic of charge carriers and carrier excitation) is largely dependent on the structure and characteristics of the individual molecules, as opposed to the crystalline structure of the lattice found in traditional inorganic semiconductors.

\subsubsection{Energy Levels of Organic Semiconductors}

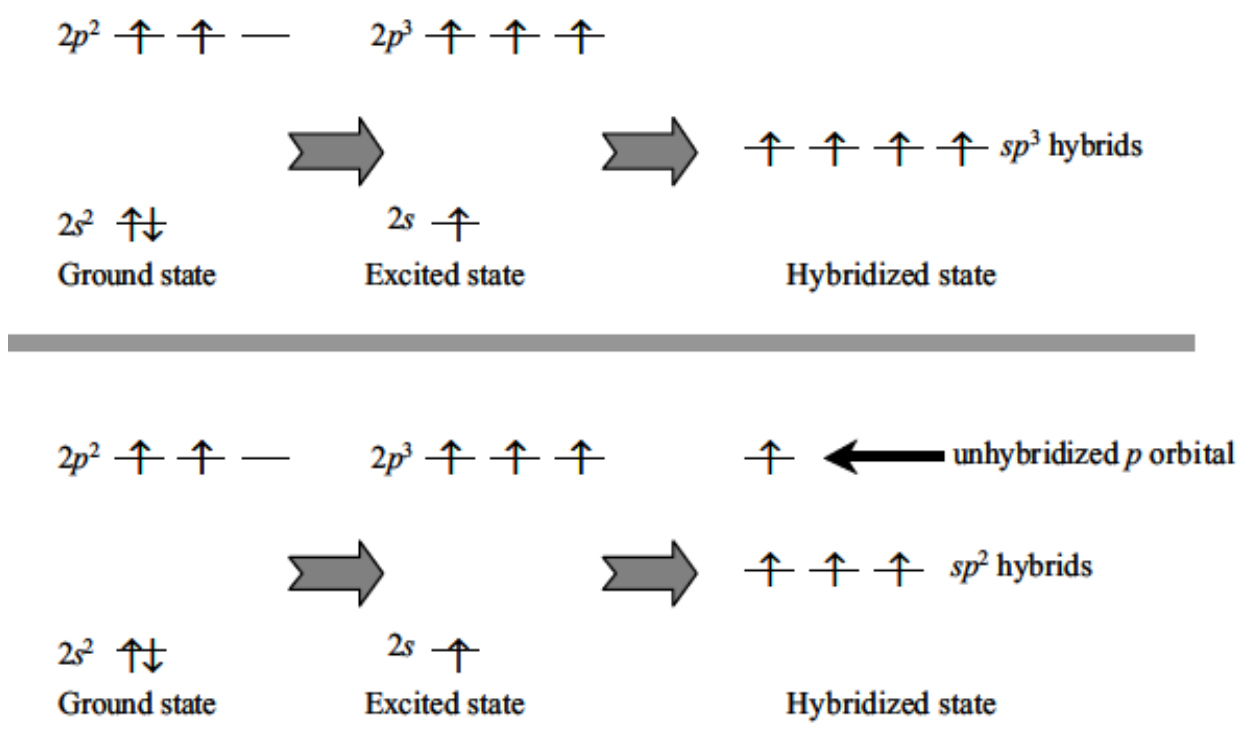

Figure 2.2 Hybridization of the valence shell electrons of a carbon atom. The upper and lower panel show $\mathbf{s p}^{3}$ and $\mathrm{sp}^{2}$ hybridization respectively [43] 
Carbon has a ground state electronic configuration of $1 \mathrm{~s}^{2}, 2 \mathrm{~s}^{2}$ and $2 \mathrm{p}^{2}$. This configuration allows carbon to form two possible hybridizations $-\mathrm{sp}^{2}$ and $\mathrm{sp}^{3}$ (Figure 2.2). $\mathrm{Sp}^{3}$ hybridization allows carbon to form a tetrahedral structure having valence of four thereby forming four covalent bonds. With this type of structure, the organic materials appear to be insulating. (For example, polyethelene). In contrast, with $\mathrm{sp}^{2}$ hybridization, carbon forms hexagonal covalent bonds, giving rise to the conjugated or semiconducting organic molecules, such as polyacetylene. Three $\mathrm{sp}^{2}$ hybridized orbital forms sigma-bonds ( $\sigma$-bonds), whereas one unhybridized $\mathrm{p}_{\mathrm{z}}$-orbital that lies perpendicular to the $\mathrm{sp}^{2}$ plane forms the pi-bonds ( $\pi$-bond). $\sigma$-bonds set up the back bone of the material whereas $\pi$-bonds enable electrical conductivity.

A Linear combination of the directed covalent bonds ( $\sigma$-bonds) from each repeating unit forms a low energy bonding sigma band ( $\sigma$-band) and a high energy anti-bonding sigma band ( $\sigma^{*}$-band) (Figure 2.3 (a)), holding the molecule together. The splitting of $\sigma$ and $\sigma^{*}$ bands is about $10 \mathrm{eV}$. Similarly, A linear combination of un-hybridized $\mathrm{p}_{z}$ orbitals forms low energy bonding pi-band ( $\pi$-band) and a high energy anti-bonding pi-band ( $\pi^{*}$-band). The energy levels of the bonding and anti-bonding pi-bands lie in between those of the $\sigma$-band and $\sigma^{*}$-band (Figure $2.3(b))$.

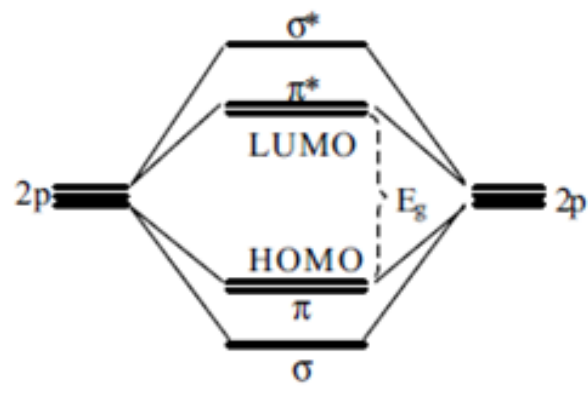

(a)

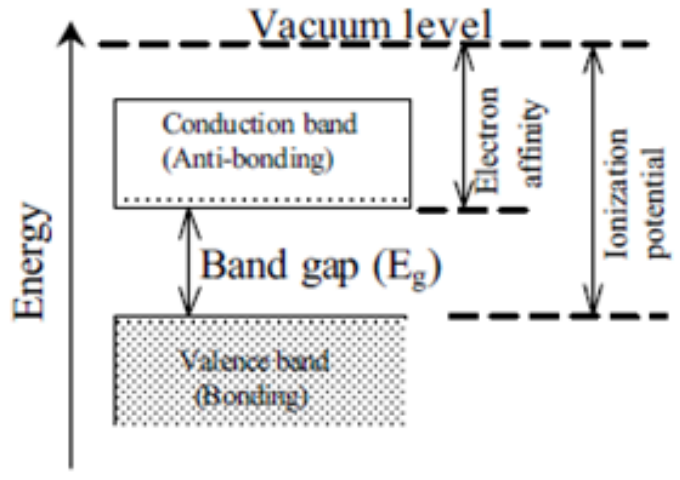

(b)

Figure 2.3 Energy level splitting of orbitals in a conjugated polymer according to molecular orbital theory (a) HOMO and LUMO level referring to the $\pi$ and $\pi^{*}$ bands (b) Collection of molecular orbitals forming bands 
separated by an energy gap [44].

The higher energy anti-bonding $\pi^{*}$-orbitals form the conduction band whereas the lower energy bonding $\pi$-orbitals form the valence band of the material. The two bands are separated by a material specific energy gap known as a band gap (Eg). The two separated bands are characterized by two quite important energy levels, namely, the electron affinity and ionization potential. The Electron affinity corresponds to the lowest state of the conduction band ( $\pi^{*}$ state), known as the lowest unoccupied molecular orbital (LUMO) in organic materials. Likewise, the ionization potential refers to the upper state of the valence band ( $\pi$ state), known as the highest occupied molecular orbital (HOMO) in organic materials. The band gaps of typical $\pi$-conjugated molecules and polymers determined from optical and other spectroscopic measurements are within the semiconducting range of 1 to $4 \mathrm{eV}$.

Unlike in inorganic semiconductors, electrons and holes in organic semiconductors are bound together by the Coulomb force. This bounded pair of electron and hole carrying no net charge is called an exciton. The Low dielectric constant of organic materials gives rise to a strong binding energy to the exciton. Due to a small wave function overlap of electrons and holes, the recombination of the exciton is slow. This leads to a long lifetime of the exciton, on the order of nanoseconds. When excitons in these materials recombine radiatively within their lifetime, luminescence occurs.

\subsection{OLED Structures}

The device structure of early OLEDs was very simple, consisting of only a single organic layer between the anode and the cathode. One example was the first PLEDs demonstrated by Burroughes et al. [45], which involved a single layer of PPV. The quantum efficiency of the PLEDs was only $\sim 0.05 \%$. Nowadays, the structure of advanced OLEDs has become more complicated, especially in small-molecule OLEDs fabricated by thermal vacuum evaporation. The 
multilayered OLEDs can consist of as many as five different organic layers situated between two electrodes. The layers typically include a hole injection layer (HIL), hole transport layer (HTL), emitting layer (EML, electron transport layer (ETL), and electron injection layer (EIL) (Figure 2.4).

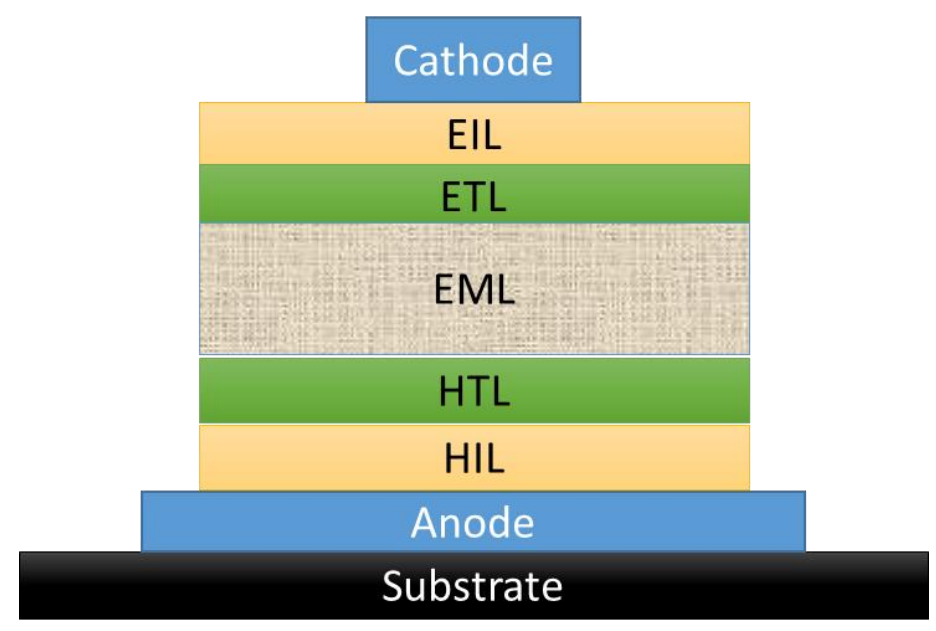

Figure 2.4 Basic multi-layered structure of OLEDs

The organic materials are typically classified according to their functions. The HIL (EIL) is the buffer layer between the anode (cathode) and adjacent HTL (ETL), which reduces the hole (electron) injection barrier and facilitates charge injection. Poly(3,4-ethylenedioxythiophene): poly(4-styrenesulfonate) (PEDOT:PSS), $\mathrm{WO}_{3}$ and $\mathrm{MoO}_{3}$ are three typical hole injection materials [46-48]. LiF and CsF are two widely-used electron injection materials [49-50].

The HTL (ETL) rapidly transports the injected holes (electrons) to the recombination zone, which is located within the EML, so the hole transport materials or electron transport materials are designed to have a high hole or electron mobility $\mu_{\mathrm{h}}$ and $\mu_{\mathrm{e}}$, respectively. N,N'bis(naphthalen-1-yl)-N,N'-bis(phenyl)-2,2'-dimethylbenzidine (NPB) and 4,4'-Bis(N-carbazolyl) -1,1'-biphenyl (CBP) are good hole transport materials [51]; tris-(8-hydroxychinoline) aluminum $\left(\mathrm{Alq}_{3}\right)$ and 4,7-diphenyl-1,10-phenanthroline (TPBi) are good electron transport materials [52].

The emitting layer usually contains a host and a dopant, which determines the wavelength of the light. Such devices integrate dopant emitters inside an emitting host matrix for the EML. 
The key benefit of the host-guest system is that several synergistic pathways for electron-hole recombination can take place. A primary pathway involves the migration of charge carriers into the EML to form excitons directly on the host matrix with subsequent energy transfer to a more efficient dopant emitter compound. An alternative process starts when one of the charge carriers becomes trapped on the emitter compound with subsequent recombination taking place on the emitter. Careful selection and ratio of the host and dopant molecules is crucial to control the overall electroluminescent properties of the EML. By changing the emitter materials, the color of the OLEDs can be varied from UV to red, covering the whole visible range.

Suitable substrate materials include glass, plastic, rigid or flexible materials. Typically, transparent conductive oxides are used as the anode materials which let the light pass. The injection and transport layers allow charge carriers passing through and recombine in emitting layers. A metal film based on low-work function $\mathrm{Al}, \mathrm{Ca}$, or $\mathrm{Mg} / \mathrm{Ag}$ are used as the cathode.

Many advanced OLED architectures have been reported, which are briefly described below.

\section{1) Top-emitting OLEDs (TEOLEDs)}

Conventional OLEDs are mostly bottom-emitting, i.e., the light is emitted through the transparent substrate and transparent or semi-transparent bottom electrode. In TEOLEDs, the light is emitted through the transparent or semi-transparent top electrode [53]. TEOLEDs are better suited for active-matrix applications as they can be more easily integrated with a nontransparent transistor backplane.

\section{2) Transparent OLEDs (TOLEDs)}

TOLEDs use transparent or semi-transparent electrodes. They can greatly improve contrast, making it much easier to view displays in bright sunlight [54]. This technology can be used in head-up displays, smart windows or augmented reality applications. Novaled's OLED panel presented in Finetech Japan 2010 boasts a transparency of 60-70\% [55].

\section{3) Inverted OLEDs}


In contrast to a conventional OLED, in which the anode is placed on the substrate, an inverted OLED uses a bottom cathode that can be connected to the drain end of an n-channel TFT, in particular for low cost amorphous silicon TFT backplanes used in the manufacturing of active matrix OLED (AMOLED) displays [56].

\section{4) Stacking and tandem OLEDs}

The concept of stacking and tandem OLEDs is to stack a set of complete OLED units on top of each other. From an electrical viewpoint, this means that several independent OLEDs are connected in series. By doing this, the current efficiency of the device is increased because one injected charge can generate multiple photons in a stack of several OLEDs instead of only obtaining at most one photon in conventional OLEDs. And the improved current efficiency leads to longer useful lifetime. The power efficiency of such a device can be higher than of a conventional device [57-58].

\subsection{Operation Principle of OLEDs}

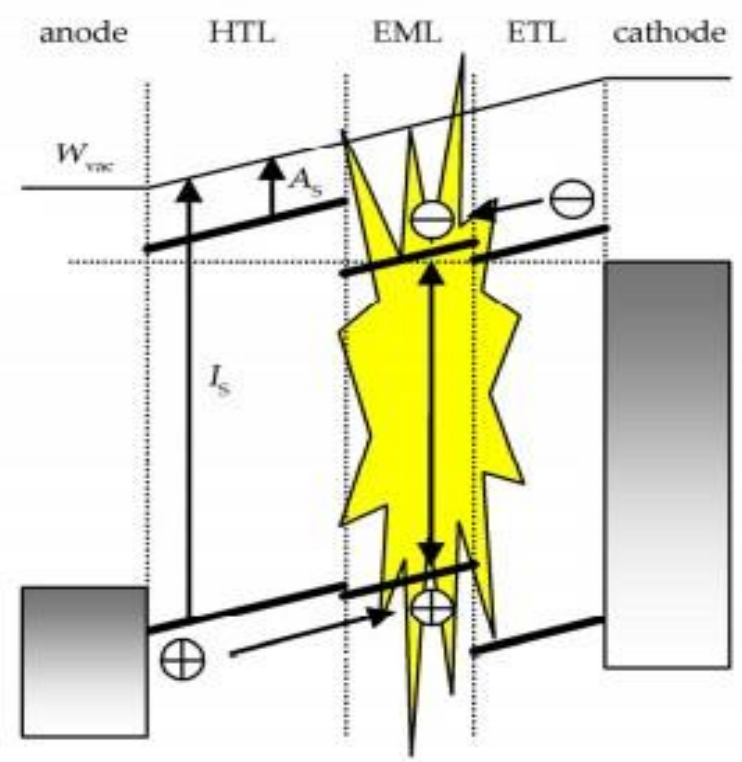

Figure 2.5 Schematic diagram of OLED operation[49] 
During operation, a voltage is applied across the OLED such that the anode is positive with respect to the cathode (Figure 2.5). A current of electrons flows through the device from cathode to anode, as electrons are injected into the LUMO of the ETL at the cathode and withdrawn from the HOMO of the HTL at the anode. This latter process may also be described as the injection of holes. Then holes and electrons drift through the organic layers toward each other under the influence of the external electric field. Some of these carriers recombine to form excitons. This happens in the EML and usually closer to the EML/ETL interface, because in organic semiconductors holes are generally more mobile than electrons. Some exciton decay routes are radiative, leading to light emission. The photon energy $\mathrm{E}_{\text {photon }}$ depends on the band gap $E_{g}$ of the emitter material, in this case the difference in energy between the HOMO and LUMO. Therefore, basically, light emission from OLEDs is governed by three major electronic processes: charge injection, transport, and recombination.

\section{1) Charge Injection}

The resistivity of a typical organic material is in the range of $10^{15}-10^{20} \Omega \cdot \mathrm{cm}$ at low electric fields $\left(<10^{4} \mathrm{~V} / \mathrm{cm}\right)$, which is too high to be considered as a good electric conductor, even semiconductor. This is the reason that the total thickness of the organic layers in OLEDs usually is $\sim 100 \mathrm{~nm}$. This extremely low conductivity also implies that organic semiconductors intrinsically have virtually no free charge carriers, so charge carrier injection is one major step in charge transport in OLEDs. Inefficient injection or extraction of charge would hamper the device performance. In general, there are three major theoretical approaches involved to describe the charge injection mechanism:

1) Field-assisted thermionic injection in which the carriers from the electrodes are thermally excited to overcome the potential barrier resulting from the superposition of the image charge potential and external field [59]. At high temperatures or low injection barrier heights, thermionic emission predicts the injection of a charge carrier from a metal contact into a semiconductor if the thermal energy of the carrier is greater than the Schottky barrier height. 
2) The Fowler-Nordheim (FN) tunneling injection model, in which the carriers tunnel through the potential barrier of the metal-organic (MO) contact under a high electric field [60]. At high electric fields or high injection barrier heights, the $\mathrm{FN}$ model describes tunneling currents through a triangular barrier into a delocalized conduction band.

3) The thermoactivated hopping injection model, which is attributed to the hopping of carriers from the metal Fermi level into the localized states of the organic semiconductor [61-62]. The results from the model were found to successfully describe the temperature and injecting contact-dependent current-voltage characteristics in a polytetraphenylbenzidine (PTPB).

In all of these approaches, the injection process is dominated by the charge injection barriers at the interfaces between the organic layers and the metal electrodes. Injection barriers can be difficult to estimate from the work function of the metal electrode and the HOMO (or LUMO) of the organic materials. Actual injection barrier heights can deviate quite strongly from the expected values. Those deviations are attributed to chemical reactions between the metal and semiconductor leading to interface dipoles [63-64], band bending [65-66], or Fermi level pinning [67]. As a rule of thumb, the currents in organic devices with injection barriers greater than $0.25-0.3 \mathrm{eV}$ at zero field are found to be injection limited, i.e., the maximum current is determined by the injection process of the charge carriers into the device, as opposed to bulk limited (or space-charge limited) devices.

\section{2) Charge Transport}

Space charge limited current (SCLC): In modern OLEDs, standard EL operation requires injection $>3 \mathrm{~mA} / \mathrm{cm}^{2}$. However, the carrier mobility is low, being $10^{-6}-10^{-4} \mathrm{~cm}^{2} /(\mathrm{V} . \mathrm{s})$ for electrons and $10^{-5}-10^{-3} \mathrm{~cm}^{2} /($ V.s $)$ for holes. Such strong injection into low mobility materials inevitably leads to charge accumulation in organic materials. The Mott-Curney relation, also known as Child's law, for trap-free unipolar conduction follows:

$$
J=\frac{9 \varepsilon \mu V^{2}}{8 L^{3}}
$$


Where $\mu$ is the mobility, $\varepsilon$ is the dielectric permittivity, $V$ is the applied voltage, and $L$ is the thickness of the sample. In the presence of shallow traps, the mobility can be thought of as "reduced effective mobility" with a pre-factor determined by the concentrations of the free and trapped charges.

$$
\begin{aligned}
& J=\frac{9 \varepsilon(\theta \mu) V^{2}}{8 L^{3}} \\
& \theta=\frac{P_{f}}{P_{t}}
\end{aligned}
$$

$\mathrm{P}_{\mathrm{f}}$ is the free carrier concentration and $\mathrm{P}_{\mathrm{t}}$ is the trapped carrier concentration. It should be noted that SCLC dominates at high current, where traps are filled and the electrode/organic contact is Ohmic or quasi-Ohmic. In the work of Mori et al. [68], SCLC, however, was not observed in multilayer small molecular OLEDs. It was not only attributed to failure of the Ohmic contact presumption at the electrode/organic interface, but also to the energy barriers between the organic layers. On the contrary, the SCLC model is easily accepted in much simpler structures, such as polymer LEDs and single layer hole-only small molecular devices.

Trap charge limited current (TCLC): Disorder, electron-phonon interaction, low bandwidth, impurities, dopants, and degradation products can all be sources to trap the carriers. The distribution of trap energies is assumed to be Gaussian or exponential. Before the trap-free SCLC limit is reached, the bulk conduction is limited by trap filling of the carriers. Burrows et al. [69] modeled the current density-voltage curve with an exponential trap distribution below the LUMO states, with a characteristic energy $E_{t}=0.15 \mathrm{eV}$ below the LUMO of $\mathrm{Alq}_{3}$. The IV relation is given by

$$
J \propto \frac{V^{\frac{T_{t}}{T}+1}}{d^{\frac{2 T_{t}}{T}+1}}
$$

Where $T_{t}=E_{t} / k$ is the characteristic temperature of the trap distribution. If $\mathrm{Tt} \gg \mathrm{T}$, the 
measurement temperature, we can assume that the electrons are full below the electron quasiFermi level and empty above it. From temperature measurements, Tt was determined to be 1780 $\mathrm{K}$, thus most of the traps are indeed deep traps as required by the model. The trap density was found to be $3 \times 10^{18} \mathrm{~cm}^{-3}$, seven orders of magnitude larger than the estimated thermally generated carrier density. Calculations showed that the nature of the traps could be structural relaxation of the $\mathrm{Alq}_{3}$ anion, which forms polaronic excited states.

Over the full range of applied bias, the IV characteristic may go through Ohmic, injection limited, shallow-trap TCLC, and trap free SCLC. At very low fields, the Ohmic conduction due to the thermally generated carriers, may be important.

\section{3) Charge Recombination}

Recombination of charges, formation of excitons and the emission of light is the final process in the OLED. Excitons are electron-hole pairs that are formed when these two species meet in the EML of an OLED. Light emission results from these excitons when they radiatively relax from their excited state to ground state. Electrically injected charges will form excitons in a 1:3 mix of singlet excitons (anti-symmetrical spin) and triplet excitons (symmetrical spin) and for fluorescent OLEDs only singlet excitons are generated in the EML whereas for phosphorescent OLEDs both singlet and triplet excitons are generated in the EML.

1) Singlet excitons - When an exciton is formed without the spin inversion of a transition electron, then the net spin of the exciton is zero. These types of excitons are known as singlet excitons.

2) Triplet excitons - When there is spin inversion during the transition of an electron, then the net spin of the formed exciton is one. Such excitons are known as triplet excitons.

A Simplified, Jablonski diagram in Figure 2.6 shows various competing processes involved during exciton generation and recombination. Electrons from higher singlet states $\left(\mathrm{S}_{2}\right.$, $S_{3} \ldots$ ) relax down to the lowest singlet excited state $S_{1}$ in a time scale of femtoseconds through an Internal Conversion (IC) process. This transition occurs without spin inversion of an electron. Once the electrons are in the $S_{1}$ states, one of two processes, either Fluorescence or Intersystem 
Crossing (ISC), can occur. The radiative decay between the lowest excited singlet state $\mathrm{S}_{1}$ and the ground state $\mathrm{S}_{0}$ causes fluorescence. Fluorescence of singlet excitons is observed in the time scales of $\sim 10^{-9}$ to $10^{-8} \mathrm{sec}$. ISC is the transition of excitons from $\mathrm{S}_{1}$ to $\mathrm{T}_{1}$ states with the reversal of electron spin at a rate of picoseconds. As a result, triplet excitons are formed. Radiative decay between the lowest triplet state $\mathrm{T} 1$ and the ground state $\mathrm{S}_{0}$ causes phosphorescence, whose time scale is much longer, on the order of $\sim 10^{-6}$ to 10 seconds.

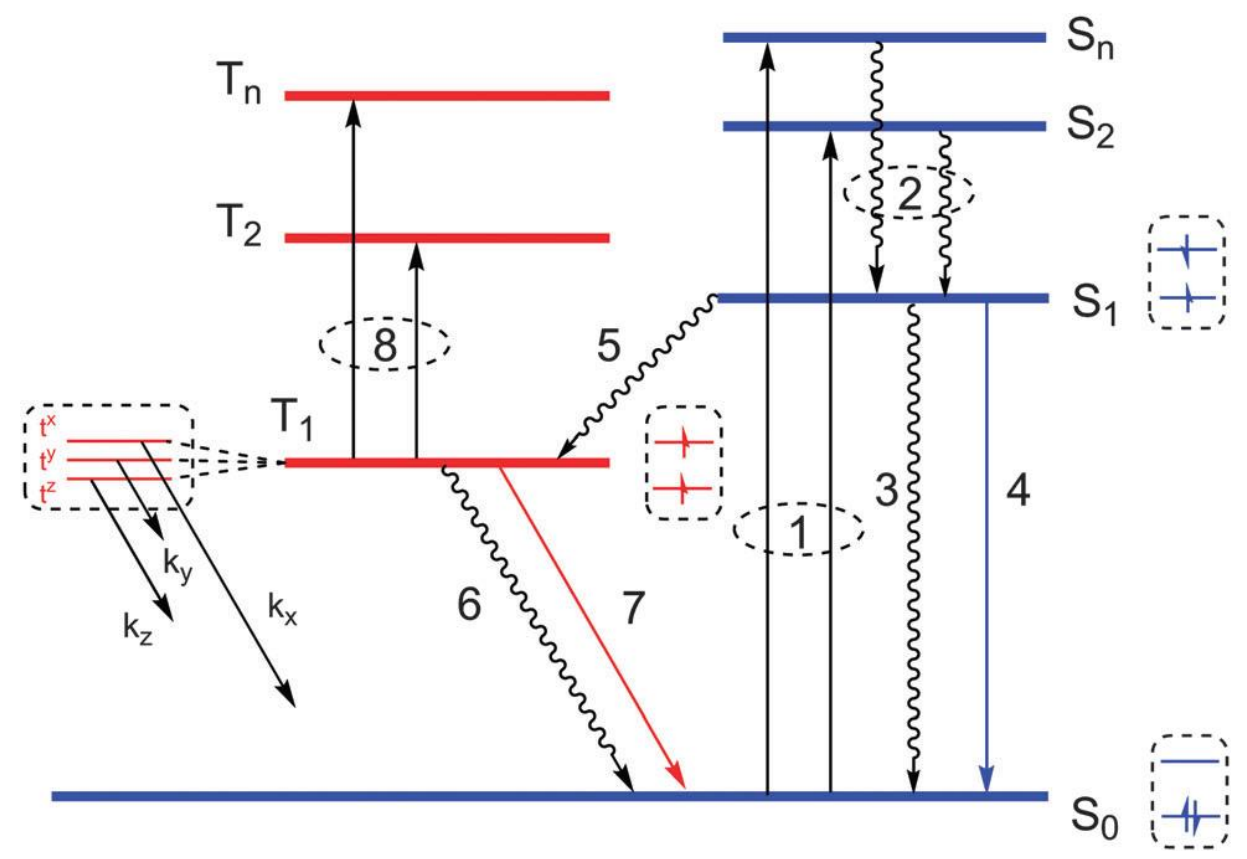

Figure 2.6 Jablonski diagram showing electron spin, absorption, fluorescence, phosphorescence and intersystem crossing [57].

However, it is more favorable when the spin-orbital coupling (SOC) is considered in some special molecular complexes. In quantum mechanics, spin-orbital coupling is the phenomenon of interaction between the spin and the motion of a particle. The spin orbital interaction is proportional to $\mathrm{Z}^{8}$, where $\mathrm{Z}$ is that atomic number of the atom; therefore, phosphorescence is favorable in the organo-metallic complexes containing heavy metal atoms such as Iridium, Platinum etc.

In order to compel the triplet excitons to emit light (to compete with non-radiative deactivation at room temperature) and to do useful work in OLEDs. It is necessary to incorporate 
special organometallic dyes into the organic materials, containing heavy transition metals, which will participate in the charge carrier recombination and provide a strong SOC in order to overcome spin-prohibition of the $\mathrm{T}_{1}-\mathrm{S}_{0}$ transition. Therefore, the problem of SOC-induced mixing of the triplet and singlet states is crucial for efficient PhOLED devices and the IQE can theoretically approaches $100 \%$ harvesting, as compared to an upper limit of $25 \%$ in conventional small-molecule fluorescent OLEDs.

\subsection{Light Extraction of OLEDs}

As it is discussed previously, the IQE of OLEDs can reach near 100\% through harvesting both singlet and triplet molecular excitation states using electro-phosphorescent materials, which is nearly fourfold increase in efficiency as compared to fluorescent organic materials. The EQE $\eta_{\text {ext }}$ (ratio of the total number of photons emitted by the OLED into the viewing direction to the number of electrons injected into organic emitter) of an OLED device is related to the IQE $\eta_{\text {int }}$ and the out-coupling efficiency $\eta_{\text {coupling }}$ (the ratio of the total number of photons coupled out in the forward direction to the number of injected electrons) by the following relation [70]

$$
\eta_{\text {ext }}=\eta_{\text {int }} \eta_{\text {coupling }}
$$

Despite achieving near $100 \%$ IQE, the external coupling efficiency of the conventional OLED device is very low. Assuming isotropic emission in the organic layer and a perfectly reflecting cathode, the fraction of generated light escaping from the substrate is [71]

$$
\eta_{\text {coupling }}=\frac{1}{\xi n^{2}}
$$

Where $\mathrm{n}$ is the refractive index of OLED material and $\mathrm{n}$ is a constant that depends on the dipole alignment and the geometry of the OLED device. For most of the organic materials $n$ is about 1.7 and taking the value of 2 for $n$, the internal coupling efficiency is only about $20 \%$. 
According to classical ray optics theory, about $80 \%$ of generated light is lost in wave-guided modes trapped inside the glass substrate or the organic structures, or emitted out from the edges of an OLED device [72] (Figure 2.7).

Many approaches that have been implemented to improve the external coupling in OLEDs by means of various internal and external device modification techniques such as, substrate modification methods, use of scattering medium, micro-lens arrays, micro-cavity, photonic crystals and nano-cavity, nano-particle, nano-wire, nano-structure and surface plasma enhanced techniques. Some of the efficient techniques are presented below.

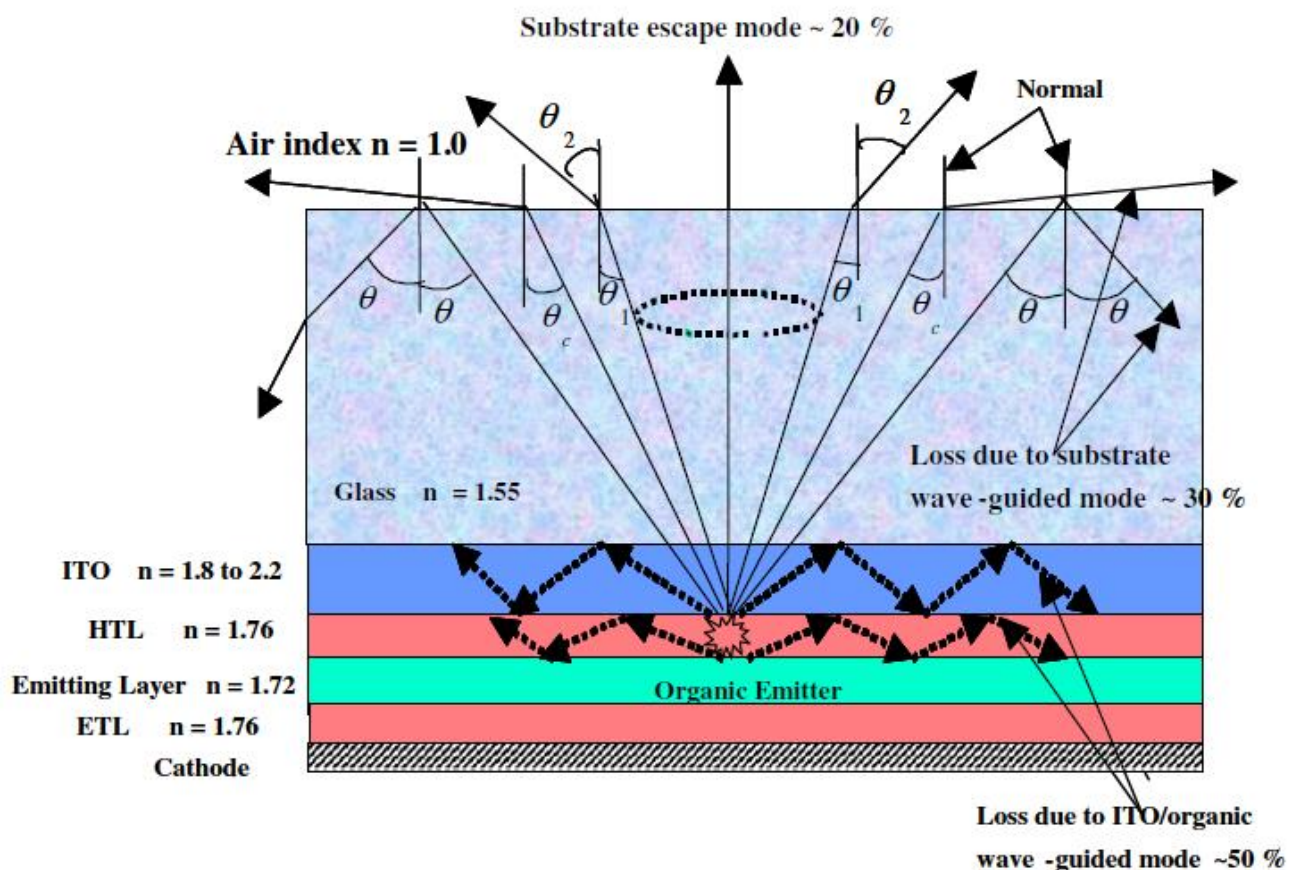

Figure 2.7 Schematic of multi-layer OLED structure and optical ray diagram of light propagation via various modes, i.e., substrate escape, substrate wave-guided mode and ITO/ organic wave-guided modes [72].

\section{1) Substrate modification techniques}

One of the simplest methods to extract substrate wave-guided mode is the application of rough surface [73]. This can be realized by sandblasting one side of the glass substrate and fabricating the OLED on the other side. Due to rough surface on the substrate, the wave-guided modes at the glass-air boundary are coupled out into air and the coupling efficiency increases on 
increasing the roughness of the substrate.

Another method for high outcoupling efficiency is texturing the meshed structures on the substrate surfaces. The textured meshed surfaces have been found to be very effective in extracting out light in inorganic LEDs [74-75]. It has been demonstrated that over $30 \%$ improvement in $\mathrm{EQE}$ can be achieved from textured surface, thin film LEDs. Enhanced external efficiency has been achieved in OLEDs by means of texturing the meshed substrates [76-77]. The textured meshed surface as shown in Figure 2.8 was fabricated using poly-dimethyl-siloxane (PDMS) using porous anodic aluminum oxide (AAO) as templates by Cheng et al [78].

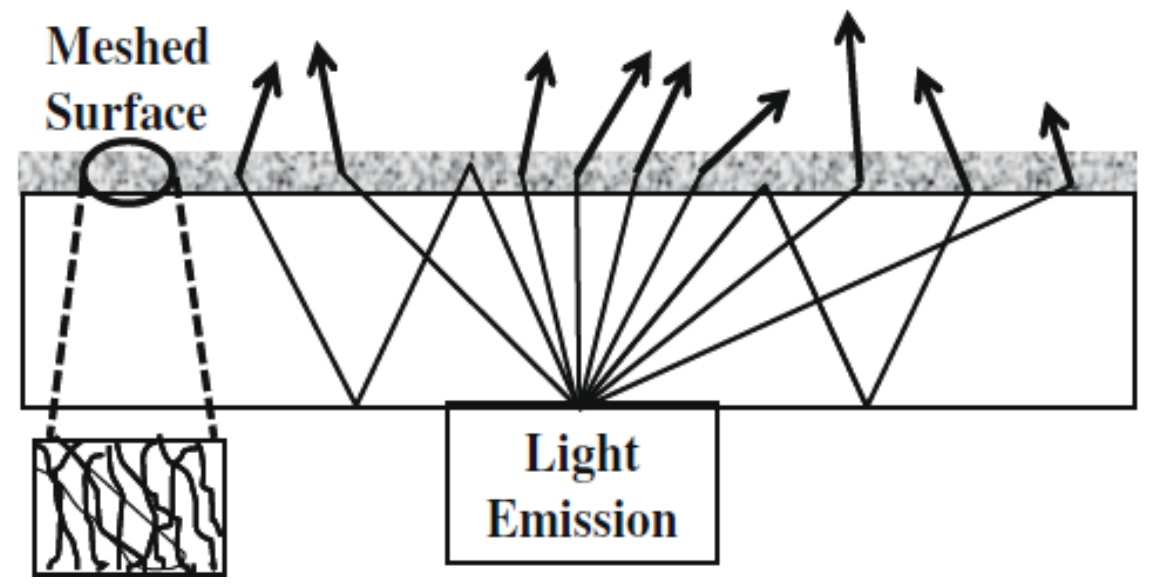

Figure 2.8 Schematic illustration of optical ray trajectories of light generated from OLED in a thin film having mesh structure on the backside of glass substrate [78]

\section{2) Light extraction by scattering medium}

In general lighting, the light extraction from OLEDs through light scattering is one of the effective choices because it offers inherent advantages, like constant color over all observation angles, symmetric illumination, and uniform and Lambertian distribution [79-80]. An ordered monolayer of silica microspheres with a diameter $550 \mathrm{~nm}$ as a scattering medium was incorporated into the conventional two-layer OLED structure by Yamasaki et al. [80]. The periodic dielectric structures consisting of hexagonally close packed silica micro-spheres array acts as two-dimensional diffraction lattice which behaves as a strong scattering medium. Silica micro-spheres were incorporated both inside the device and on the backside of glass substrate as 
shown in Figure 2.9.

This ordered array of silica micro-spheres acts as strong scattering centers and the waveguided modes into glass and ITO/organic interface could be easily coupled out and significant enhancement in the light out-coupling efficiency was observed, but some spectral changes, such as, broadening, narrowing and peak splitting of generated light is inevitable [81].

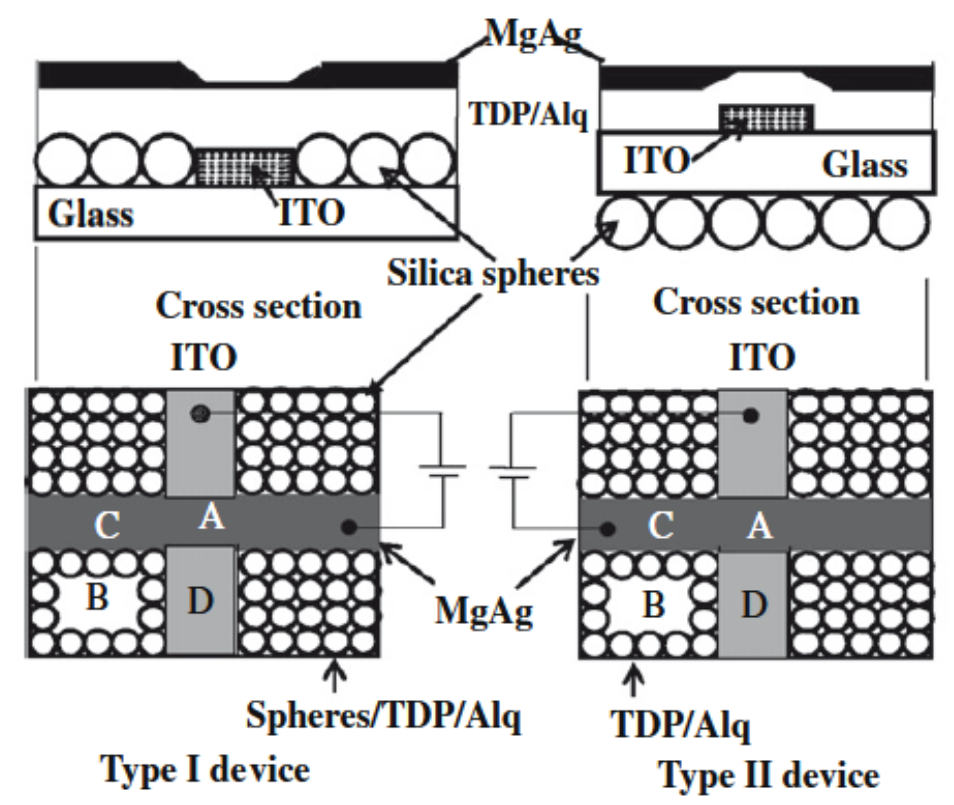

Figure 2.9 Schematic representation of OLEDs with silica micro-spheres as scattering medium [81]

\section{3) Light extraction by micro-lenses}

Use of micro-lenses on the backside of the glass substrate is one of the most effective technique for extracting out substrate wave guided modes. Using ordered micro-lens arrays, an improvement in the light out-coupling efficiency of OLEDs has been achieved [82-84]. Moller and Forrest [81] fabricated the ordered micro-lens arrays on the backside of an OLED glass substrate with $10 \mathrm{~lm}$ diameter of PDMS with refractive index $\mathrm{n}=1.4$. Figure 2.10 depicts the schematic diagram of an OLED structure with ordered micro-lenses on the backside of glass substrate. In the presence of ordered micro-lenses on the surface, the angle of incidence of light rays is smaller than the critical angle $\theta_{c}$ that leads to light extraction [81] and total internal 
reflection light glass-air boundary is coupled out. It has been demonstrated that the light outcoupling can be improved using ordered micro-lenses by a $50 \%$ increase in $\eta_{\text {ext }}$.

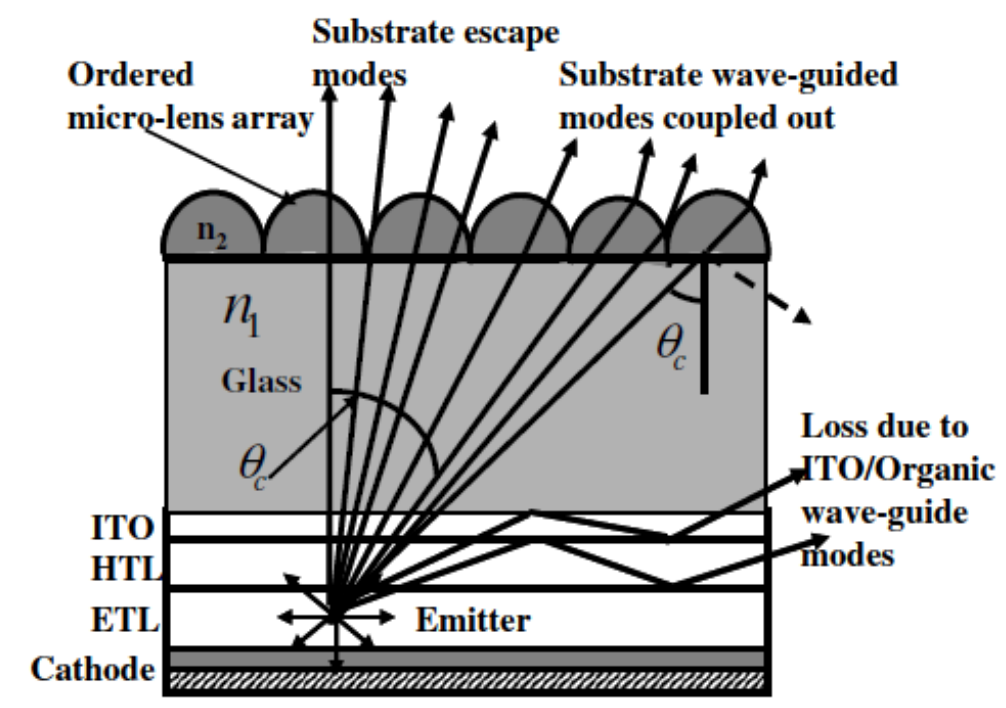

Figure 2.10 Schematic ray optical diagram with ordered micro-lenses on the backside of glass substrate [82]

\section{4) Light out-coupling enhancement by micro-cavity structure}

An OLED can be regarded as one-dimensional micro-cavity because the total thickness of organic films in the device is of the order of wavelength of light. There are two types of micro-cavities in OLED, that is, weak and strong micro-cavities [85]. A weak microcavity is formed with the conventional OLED structure due to the metal cathode and high refractive index anode (ITO) [86] while a strong micro-cavity OLED structure usually consists of a metal mirror on one side and a highly reflective dielectric multilayer structure on the other side [87]. A detailed analysis of the effect of weak micro-cavity on the OLED is given by Bulovic et al. [88]. According to this effect the conventional OLED structure acts as micro-cavity and weak reflections take place from the metal cathode and other reflective surfaces. The radiative lifetime of the molecular excited states can be strongly influenced by such micro-cavity. 


\subsection{Fabrication and Characterization of OLEDs}

\subsubsection{Fabrication Process of OLEDs}

Generally, the fabrication process of OLEDs can be categorized into two main aspects: small molecule OLEDs (SMOLEDs) based on vacuum thermal evaporation (VTE) technique and polymer OLEDs (PLEDs) based on solution-based deposition technique.

Currently, the cost of OLED display and lighting is 3 to 10 times that of LCDs and it has been suggested that the cost competitiveness of OLED displays can be improved through the use of solution-based deposition techniques. Not only does solution deposition potentially require lower capital investment, it is also compatible with large area substrates now used in manufacturing. Furthermore, it is considerably more efficient in material utilization. Since material costs are projected to be a significant expense in the manufacture of OLEDs, material utilization will be a key performance parameter.

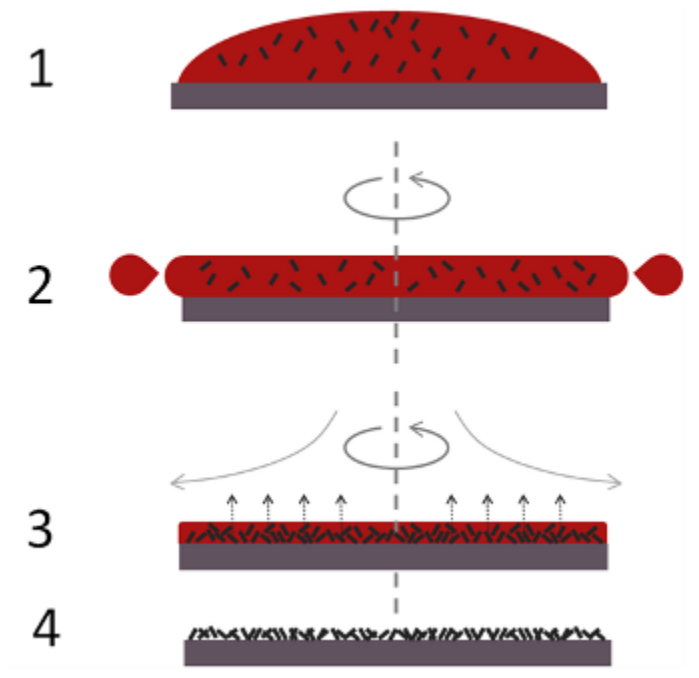

Figure 2.11 Example of spin coating a small molecule in solution using a static dispense (https://www.ossila.com/pages/spin-coating). The substrate is coated in the ink containing the molecules dissolved in a solvent (1). Then the substrate is rotated at high speed and the majority of the ink is flung off the side (2). Airflow then dries the majority of the solvent leaving a plasticised film (3) before the film fully dries to just leave the molecules on the surface (4). 
Several methods of solution deposition exist for printing organic materials onto flat substrates which are suitable for the creation of patterned multilayer OLED structures. The optimal printing method will need to be able to deliver an OLED material or mixtures of OLED materials as an ink at high throughput rates, with high feature definition, and be able to be printed as several layers on top of each other. Graure offset, flexographic, screen, and ink-jet printing are currently the most commonly used techniques in large-scale commercial printing. In lab research scale, spin coating is widely used for the preparation of small-size samples. The liquid is spread by centrifugal force, leading to a thin and uniform coating as shown in Figure 2.11. The thickness of the film depends upon the viscosity of the organic film and the spin speed.

Vacuum thermal evaporation (VTE) has been the most commonly used deposition process, since the early days of OLED development by Tang and VanSlyke at Kodak. Vacuum thermal deposition is conceptually a very simple process, where pure versions of the desired materials are evaporated from crucibles using thermal energy. Adjusting the temperature of the crucible can control the rate of evaporation, and therefore the rate of deposition. The crucibles are resistively heated to generate vapor which then propagates along a line of sight path to the substrate where it condenses to form a film. This line of sight propagation also enables simple patterning through the use of shadow mask, currently the dominant technique for both lighting and displays. While the promise of a low-cost printable display technology has been a key driver for interest in OLEDs, vacuum evaporation remains the method currently used for almost all small molecule displays and lighting in production as of 2016.

Although spin coating remains a very useful laboratory technique for the initial optimization of materials, the residual solvent and nonuniformity in the final film arising from particles or other defects can influence the performance of the devices. VTE has several advantages that have led to the dominance of this technology. The first advantage of VTE is the ability to deposit complex, multilayer device structures with almost unlimited combinations of different materials. Vacuum deposition is well suited to forming these complex structures 
because each layer can be deposited as a pure material without solvents, with precise control of thickness and lateral pattern, and with virtually no interactions with the other layers in the structure. Other deposition methods, particularly solution-based methods, typically use solvents to enable printing or coating of the organic materials. While these approaches are typically low cost (solution coating) or simple to pattern (printing) they significantly limit the device design possibilities due to the need for orthogonal solvents. Basically, each successive layer cannot use a solvent that dissolves one of the previous layers. As there are a limited number of potential solvent classes, this significantly limits the total number of device layers. It also limits the types of materials that can be used, as not all are readily soluble. For example, $\mathrm{Alq}_{3}$ is a classic and historically important OLED material with poor solvent solubility.

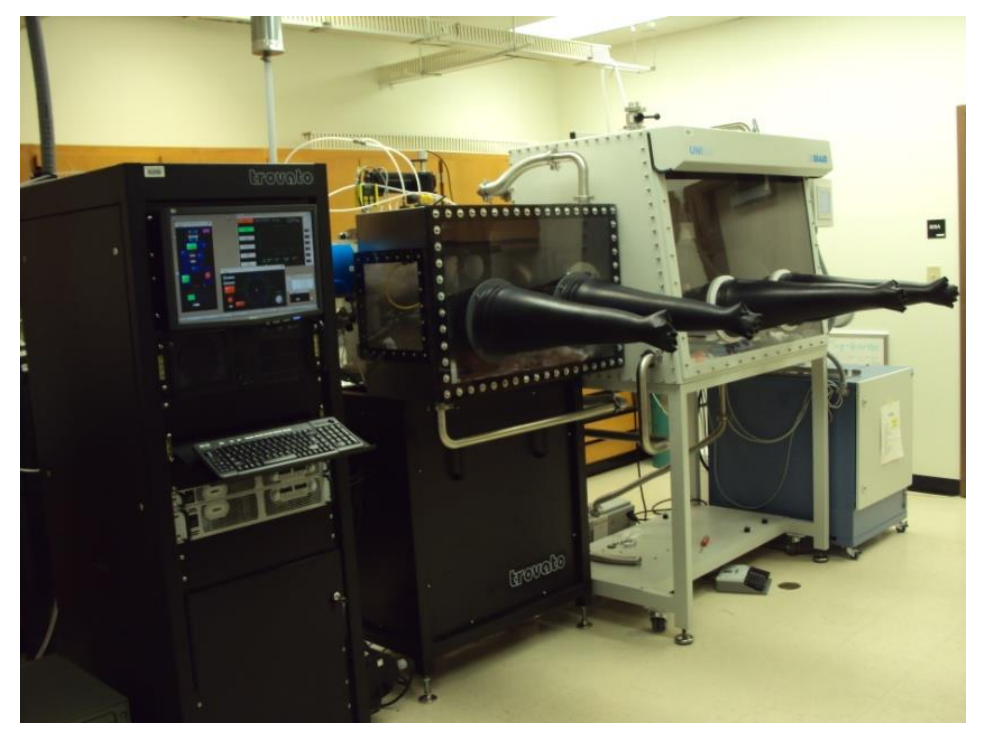

Figure 2.12 Travato Thermal Evaporation System connected to a $\mathbf{N}_{2}$ filled glovebox.

Second, VTE is one of the cleanest methods for depositing materials for a wide range of applications and cleanliness is critical to achieve high performance and long lifetime OLEDs. This cleanliness arises for two reasons. First high-purity source material is typically used to prevent degradation during evaporation. Because any material to be deposited using VTE must sublime at appropriate pressures and temperatures, zone sublimation refining can be and is usually used to purify source materials. Second, environmental contamination is absent in the 
high-vacuum environment used in most VTE systems. Most OLED materials are highly sensitive to oxygen and water vapor and, while inert gas environments can be used to provide some protection, high vacuum is typically the best. This vacuum environment not only significantly reduces the potential for contamination species, it also helps to minimize particle contamination during device fabrication. The thin layers (10-100 nm typically) used in standard OLED designs are especially susceptible to particulate damage, which leads to shorts. In the vacuum environment, there are no air currents to carry particulates, vacuum does reduce the likelihood of contamination.

\subsubsection{Characterization of OLEDs}

\section{1) Current density-voltage (J-V) Analysis}

Like inorganic LEDs, a very basic and yet very simple measurement for OLEDs is current density-voltage $(\mathrm{J}-\mathrm{V})$ curve as it directly shows if the device is working properly and gives a first impression on its quality.

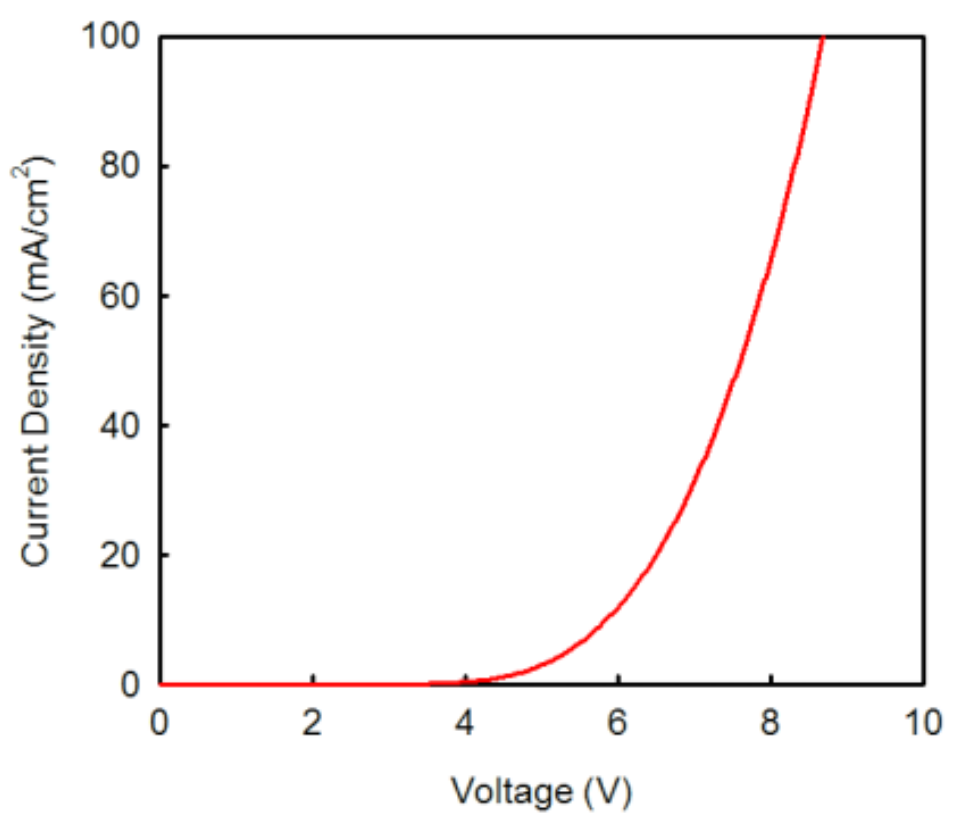

Figure 2.13 A typical J-V curve of an OLED device 
A bias voltage sweep is applied to the device and its current response is recorded and then the current density is obtained with fixed active area. A typical J-V curve is shown in Figure 2.13, where current-voltage measurements were made using an Agilent $4156 \mathrm{C}$ semiconductor parameter analyzer and the current was measured as a function of the applied voltage. The test set up was connected to a computer where the software was used to control the testing parameters. Overall ohmic resistance is attained from $\mathrm{J}-\mathrm{V}$ curve and from Equation 2.2, the higher the operation voltage is at a constant current density, the lower the carrier concentration is.

\section{2) Electroluminescence (EL) Spectra Measurement}

Electroluminescence is an optical phenomenon and electrical phenomenon in which a material emits light in response to the passage of an electric current or to a strong electric field. EL of OLEDs has the same principle like inorganic LEDs. The current is fed through electrode and electron hole pairs are formed in EML where luminescence occurs. In addition, EL also reflects the sensitivity of an OLED device to the human eye. Figure 2.14 shows a typical white OLED device with 3 different optical peaks, representing blue, green and red emission peaks. In this dissertation, the current is injected by a Keithley sourcemeter and the emission spectra is recorded using an Ocean Optics spectrometer.

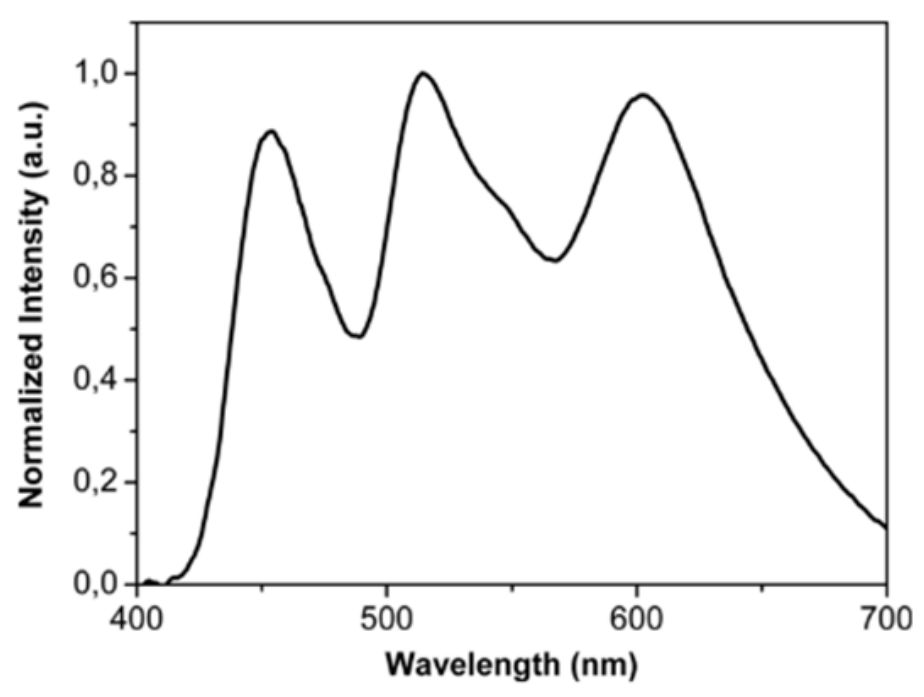

Figure 2.14 Typical white OLED spectrum is generated by three different emitters (blue, green, and red). 


\section{3) Luminance-Current Density (L-J) and Luminance-Voltage (L-V) Measurement}

The luminance of OLED devices is firstly measured by placing the device on top of a calibrated silicon photodiode and is swept with voltage from a Keithley sourcemeter. All the emitted photons are then captured by the photodetector and are converted into photocurrent. In addition, photocurrent can be converted to luminance $\left(\mathrm{cd} / \mathrm{m}^{2}\right)$ by multiplying a constant. An alternative way to read threshold voltage $V_{t}$ is from $L-V$ curve. Since most OLEDs emit in the visible spectrum, the photonic response, also called human eye response, directly affects the luminous efficiency of the device. The luminous efficiency, $\eta_{L}$, in candela per amperes $(\mathrm{Cd} / \mathrm{A})$, weighting the photons according to the photonic response of the eye can be directly calculated by L-J curve. Mathematically, $\eta_{\mathrm{L}}$ is defined as,

$$
\eta_{\mathrm{L}}=\mathrm{L} / \mathrm{J}
$$

where, $\mathrm{L}$ is the brightness in $\mathrm{Cd} / \mathrm{m}^{2}$ and $\mathrm{J}$ is the current density in $\mathrm{A} / \mathrm{m}^{2}$ of the OLEDs.

\section{4) Lifetime Measurement ( $L-T$ curve)}

Typically, the degradation of OLEDs manifests as a continuous loss of the device efficiency, primarily observed as the decrease of brightness at constant current or voltage. Therefore, the main parameter to evaluate the lifetime of an OLED is the behavior of the luminance over time (at a fixed or predefined physical environment, e.g., temperature, current density, voltage, and/or humidity), called the lifetime of the devices. Often, the lifetime (denoted as $\mathrm{T}_{50}$ or $\mathrm{T}_{1 / 2}$ ) is defined as the time the luminance drops to half of its initial device brightness at a constant current density. Equation 2.6 defines the relationship between the initial luminance $\mathrm{L}_{0}$ and the lifetime $T_{1 / 2}$ of a device, quantified with the constant $\mathrm{C}$. The acceleration factor $\mathrm{n}$ is material and device specified and depends therefore on the device structure and the organic materials used.

$$
L_{0} \times T_{1 / 2}=C
$$

In most cases, the degradation behavior of an OLED may be influenced by multiple independent degradation mechanisms. Thereby, the lifetime curve of such an OLED may show an 
initial rapid decay and a more moderate (long-term) luminance drop. One possible way to fit such a behavior is to combine different exponential decay functions, like it is shown from Equation 2.7, where $\mathrm{a}, \mathrm{b}, \quad a$, and $\beta$ are fitting parameters.

$$
\frac{L_{(t)}}{L_{(0)}}=a e^{-\alpha t}+b e^{-\beta t}
$$

In this dissertation, $\mathrm{T}_{80}$, which refers to the time when luminance drops to $80 \%$ of its initial value, is used to represents the initial rapid decay process and $\mathrm{T}_{50}$ reflects the overall degradation performance of an OLED device. 


\section{Chapter 3 Reliability Study of OLEDs}

\subsection{Introduction}

Over the last decade, a significant increase in OLED device lifetime and efficiency has been achieved, allowing for practical use of OLEDs in small electronics. A revenue of several billion dollars has already been achieved, mostly with OLED-based active-matrix display applications. According to IDTechEx, the OLED display market is expected to grow to about 25 billion dollars in 2017. Nevertheless, the relatively poor lifetime of OLEDs still hamper their use in the general lighting markets [89-91].

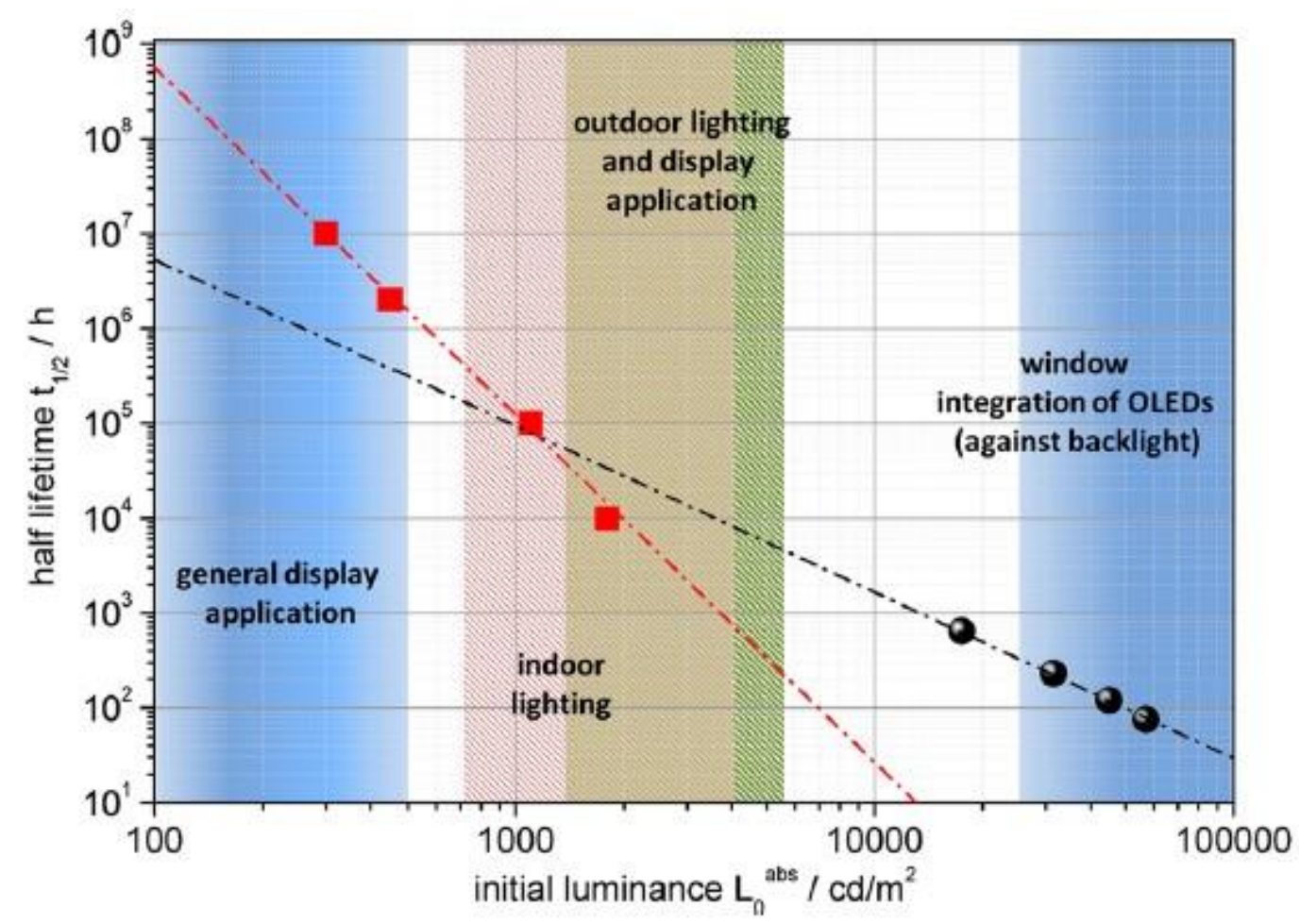

Figure 3.1 Overview of relevant OLED brightness. Current lifetime data are from the state-of-the-art red (redø) and white (๑) OLEDs from Meerheim et al. and Loser et al. respectively [92].

Typically, the higher is the luminance level, the shorter is the lifetime of an OLED. Figure 
3.1 displays the required luminance of OLEDs for certain applications together with the state-ofthe-art lifetimes of red and white OLEDs. It is obvious that for display applications, a wide range of stable materials and OLED stacks has been already discovered and is available. Even though the issue of lifetime remains, a wide range of OLED displays has been released over the past few years. For applications requiring very high brightness, like outdoor displays, HDR (high dynamic range) displays, and general lighting, the current OLED technology still does not provide the required lifetimes. Further improvement of the lifetime particularly at high brightness levels requires a greater understanding of the degradation mechanisms of OLEDs, which would pave the way for developing reliable devices for broader applications as display and solid-state lighting sources.

\subsubsection{Fundamental Mechanisms of OLED Degradation}

The degradation of OLEDs usually implies some undesirable internal processes such as chemical reactions, morphological (phase changes, crystallization, and delamination processes), and other physical (charge accumulation) changes. These processes result in various changes in device properties, most notably in the color-luminance-current-voltage characteristic of OLEDs. Typically, the degradation starts as a sharp or gradual loss of the device luminance. Therefore, the main parameter to evaluate the lifetime of an OLED is the behavior of the luminance over time (at a fixed or predefined physical environment, e.g., temperature, current density, voltage, and/or humidity), called the lifetime of the devices.

An extended overview about the known degradation mechanisms was provided by Aziz and Popovic in 2004, who focused their review on the visible appearance of the OLED degradation mechanisms. Certainly, these visible effects are (i) the immediate breakdown of the electroluminescent behavior ("catastrophic failure"), (ii) the growth of nonemissive areas, mainly known as "dark-spot degradation", (iii) the (more or less) long-term degradation effect ("intrinsic degradation"), well described by the (stretched) exponential decay function of the decreasing 
luminance, and (iv) additionally, the operational voltage increase during the aging process [93].

Within the overview about the degradation mechanisms, Aziz and Popovic discussed the influence of the morphological behavior of the aged organic semiconductors, the "Alq 3 -instabiliy model", the relevance of indium migration, as well as mobile ionic impurities and a model of immobile charge accumulation [94]. Aziz and Popovic clearly pointed out that there is an obvious difference between the lifetime behavior of devices and the luminescence decay, which may occur due to intrinsic mechanisms and extrinsic effects. The following processes are usually considered in the context of intrinsic degradation mechanisms: exciton reactions, charge carrier reactions, migration of ionic species, charge accumulation, and changes in the electric field profile due to molecular reorientation. The most commonly mentioned external causes of OLED degradation are arguably light, oxygen, water, and temperature. Some process parameters of the device production, like pressure (and therefore oxygen and water content), evaporation rate, temperature, and impurities, play an important role on device stability as well. The external influences are controllable due to improved production conditions, like an applied high vacuum in the range of $10^{-8}$ mbar, highly pure substrates and semiconducting materials, as well as the usage of the stateof-the-art encapsulation techniques.

Nonetheless, the intrinsic and extrinsic factors may overlap, and the analysis of the individual reactions may remain challenging, as shown in Figure 3.2. Until now, fortunately, a large number of fabrication process-related failure mechanisms caused by extrinsic factors, especially the "dark spot degradation", can be avoided completely by reducing extrinsic sources of degradation, including metallic impurities, residual water, and other environmental contaminants [95-96].

The intrinsic degradation, which manifests as a gradual decrease in the brightness without any obvious change in the device appearance, is more complicated and a bigger obstacle to overcome. Various mechanisms have been proposed to explain the intrinsic degradation behaviors of OLEDs, including re-orientation of dipoles, charge migration and accumulation [97], and 
electrochemical decomposition of organic materials [98-99]. The resultant products are believed to screen the local electric field or act as nonradiative recombination centers and luminescence quenchers [100]. All these processes can be thermally enhanced by joule heating generated during the driving process of OLEDs. Therefore, joule heating under a large driving current may greatly accelerate the degradation of OLEDs [101-102]. This current-induced thermal effect on the reliability of OLEDs, however, has not been fully investigated.

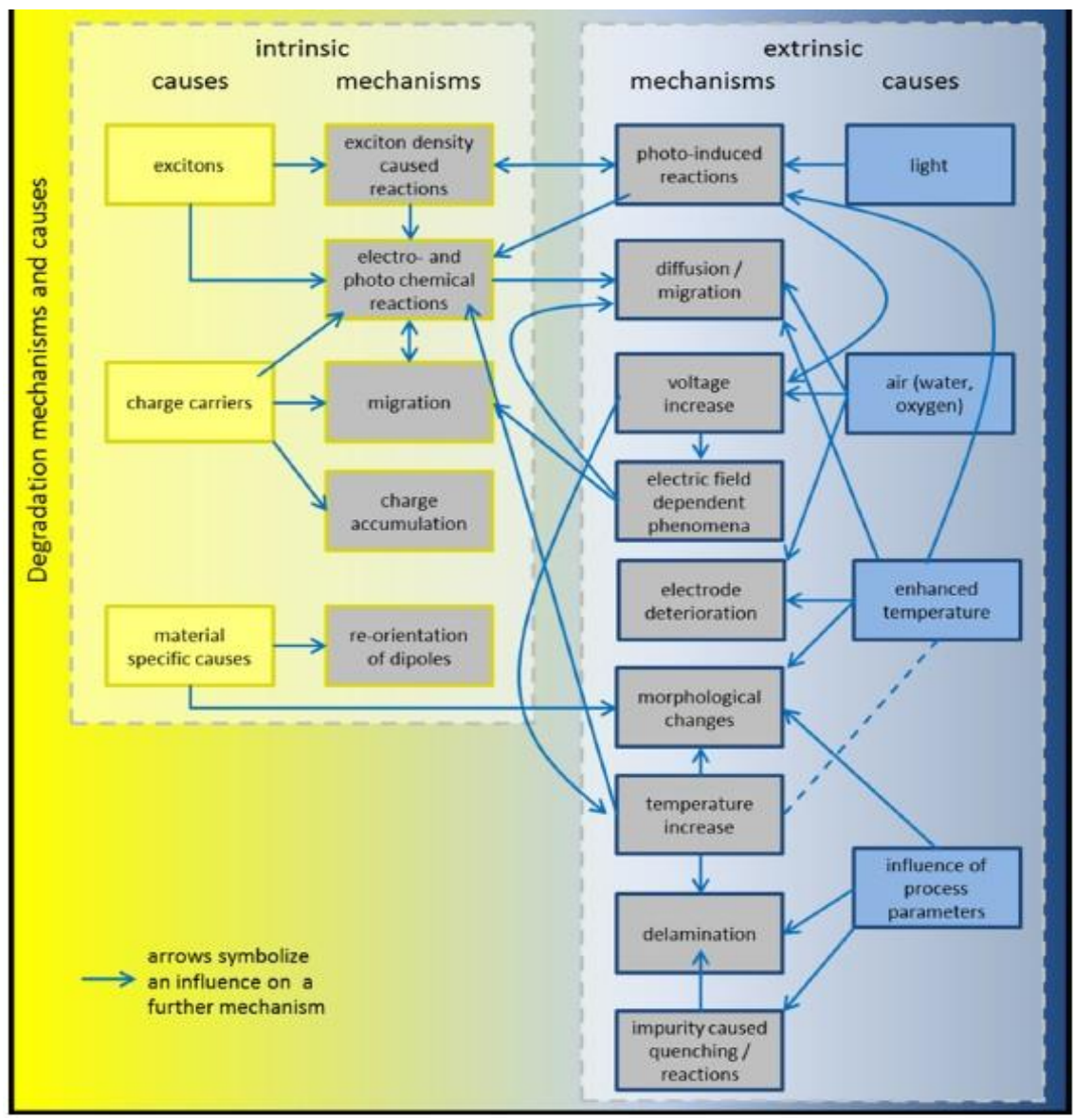

Figure 3.2 Intrinsic and extrinsic causes and mechanisms for OLED degradation [96]

\subsubsection{Research Motivation}

Most studies on the degradation effect caused by Joule heating focused from outside the device and annealing is a common method to control the temperature [103-105]. However, Joule heating usually generated in the heterointerfaces inside the stacked OLEDs structure because of 
the energy gap difference between two materials is usually ignored. This self-generated heat inside the device is hard to investigate because the amount of the heat is hard to be quantified. In addition, most previous studies on OLED reliability have investigated device degradation under constant current operation conditions, in which the heat exist during the whole working process.

In this chapter, a simple yet effective method to study the degradation effect of joule heating generated inside the OLED devices is provided. The electrical and optical degradation of green phosphorescent OLEDs under pulsed current stressing is fully investigated. In the pulsed stressing OLEDs, self-heating effects are substantially suppressed, allowing us to differentiate the thermal and nonthermal factors governing the device degradation process. The pulsed current is further modified to include a reverse bias component, equivalent to an AC power source, and its effects on the OLED lifetime are studied. Two sets of stressed OLED devices with different energy gap between injection and hole transport layers are compared to further discuss the aging effect of energy alignment between charge transport layers.

\subsection{Experimental Procedure}

Figure 3.3 shows the schematic diagram of the electric circuit. A pulse generator, a variable resistor and OLED devices are series connected and an oscilloscope is parallel connected across the variable resistor to calculate the current passing through OLED devices.

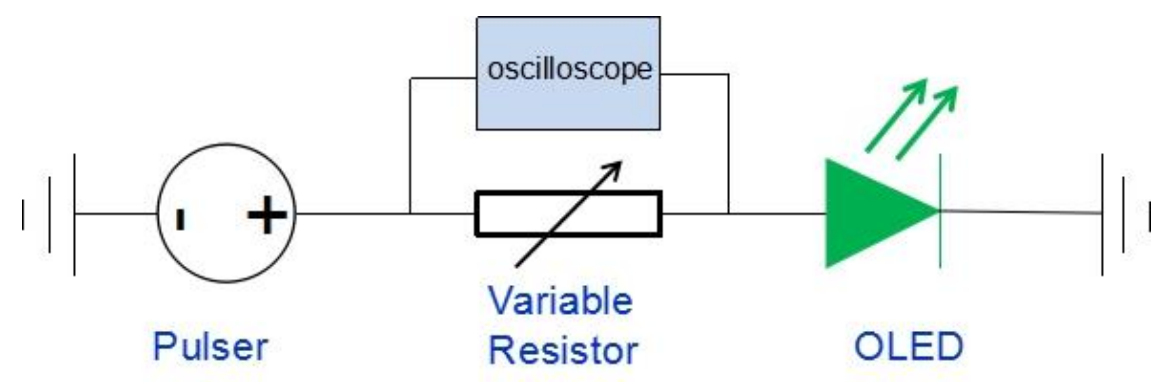

Figure 3.3 The schematic diagram of electric circuit.

By adjusting the frequency and duty cycle of the pulse generator, degradation results of OLEDs are discussed driven by pulsed currents with 10\%, 20\% and 50\% duty cycle. The short 
pulses with 10 and $20 \%$ duty cycle significantly eliminate the effect of self-heating and thus a prolonged lifetime is expected.

Green phosphorescent OLEDs were fabricated on glass substrates with pre-patterned ITO (sheet resistance $15 \Omega / \square$ ). The substrates were first cleaned with solvents and deionized water, and treated with $\mathrm{O}_{2}$ plasma for $5 \mathrm{~min}$. They were then transferred to a thermal evaporation system, where the organic layers were deposited at a pre-calibrated rate $0.1 \mathrm{~nm} / \mathrm{s}$. The OLED

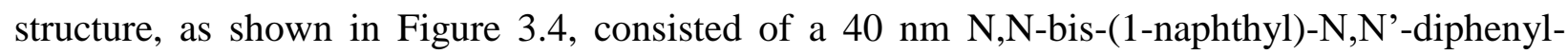
1,1'-biphenyl-4,4'-diamine (NPB) or 4,4'-N,N'-dicarbazolebiphenyl (CBP) as hole transport layer (HTL), a $30 \mathrm{~nm} \mathrm{CBP}$ doped with 7 wt.\% fac-tris(2-phenylpyridinato-N,C2') iridium (III) [Ir(ppy) $)_{3}$ light-emitting layer, and a $45 \mathrm{~nm}$ 1,3,5-tris(2-N-phenylbenzimidazolyl) benzene (TPBi) electron transport layer. Finally, a $0.5 \mathrm{~nm} \mathrm{LiF/} 100 \mathrm{~nm} \mathrm{Al}$ cathode was deposited through a shadow mask, which defined the active area of the OLEDs to be $0.1 \mathrm{~cm}^{2}$. Four identical OLEDs were fabricated on each substrate. They were encapsulated with epoxy and a glass lid in a $\mathrm{N}_{2}$ filled glovebox and characterized in air at room temperature.

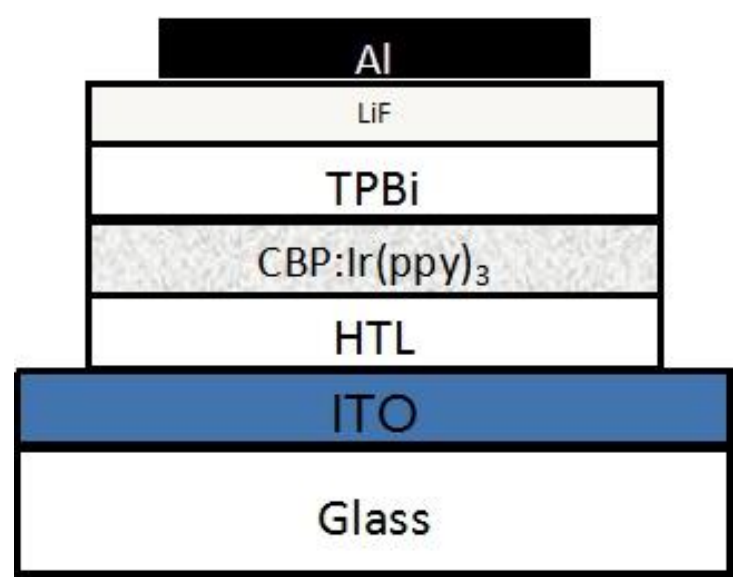

Figure 3.4 Layer structure of the green phosphorescent OLEDs and two sets of OLED devices are fabricated and tested with different types of HTL

\subsection{Results and Discussion}

To evaluate the device reliability, as-fabricated OLEDs were stressed at a constant continuous wave (CW) or pulsed current density, and luminance data was collected periodically. 
At a fixed duty cycle of $10 \%$, the dependence of the electroluminescence (EL) intensity on pulse frequency was found to be a function of the injected current density, as depicted in Figure 3.5.

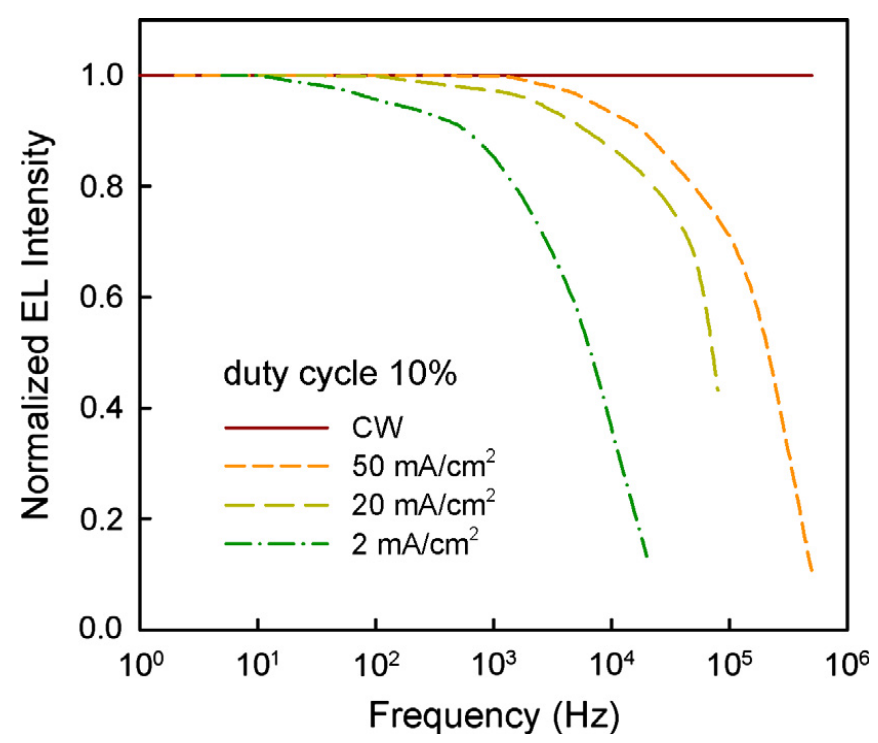

Figure 3.5 Frequency-dependent EL intensity of green phosphorescent OLEDs at different pulsed current densities.

At $2 \mathrm{~mA} / \mathrm{cm}^{2}$, the EL intensity starts to drop at $10 \mathrm{~Hz}$. The $3 \mathrm{~dB}$ bandwidth is $6.5 \mathrm{kHz}$. The bandwidth increases with increasing injection current level. At $50 \mathrm{~mA} / \mathrm{cm}^{2}$, the initial EL drop occurs at $1 \mathrm{kHz}$, and the $3 \mathrm{~dB}$ bandwidth is $200 \mathrm{kHz}$. This behavior is consistent with previous theoretical and experimental observations that both the rise and decay times of the transient EL of phosphorescent OLEDs decrease as the amplitude of pulsed voltage is increased [106-107]. By solving the dynamic equations describing the diffusion and redistribution of charge carriers, Chandra et al. have found that the rise time increases linearly with increasing ratio of the applied voltage and current density $(\mathrm{V} / \mathrm{J})$, whereas the decay time is determined by either the time constant of the OLED or the lifetime of radiative triplet excitons [108]. For the pulsed stressing conducted in this work, a current density of $50 \mathrm{~mA} / \mathrm{cm}^{2}$ much higher than the industrial standard was used and the frequency was fixed at $1 \mathrm{kHz}$, whereas the duty cycle was varied from $10 \%$ to $50 \%$. The short pulses with $10-20 \%$ duty cycle significantly reduced the total injection power, and thus largely eliminated the effects of self-heating on the OLED stability. 
The EL spectra and current density-voltage (J-V) characteristics of the OLEDs were recorded using an Ocean Optics fiber-optic spectrometer and an Agilent 4156C semiconductor parameter analyzer, respectively, before and after the stressing tests. To calculate the external quantum efficiency (EQE), the OLEDs were placed directly onto the surface of a calibrated silicon photodetector $(1 \mathrm{~cm}$ in diameter) and all emitted photons from the glass side were captured.

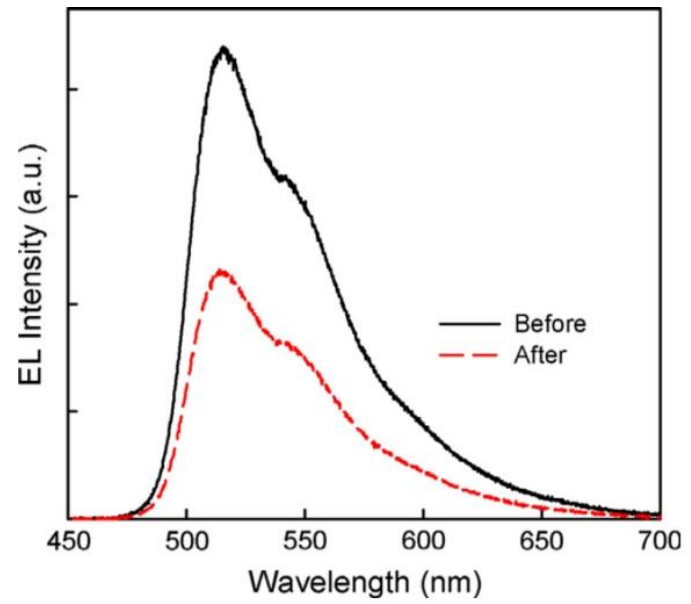

(a)

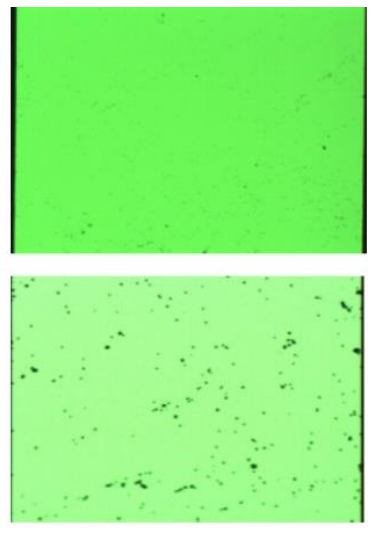

(b)

Figure 3.6 (a)EL spectra of a phosphorescent OLED before and after stressing under $50 \mathrm{~mA} / \mathrm{cm}^{2} \mathrm{pulsed}$ current $(1 \mathrm{kHz}, 20 \%$ duty cycle) for $60 \mathrm{~h}$. (b)The micrographs of the unstressed and stressed OLEDs operating at $20 \mathrm{~mA} / \mathrm{cm}^{2}$

Figure 3.6(a) displays the EL spectra of a typical OLED before and after $50 \mathrm{~mA} / \mathrm{cm}^{2}$ pulsed stressing with a duty cycle of $20 \%$ for $60 \mathrm{~h}$. The unstressed OLED shows the typical EL spectrum of $\operatorname{Ir}(\mathrm{ppy})_{3}$ with a peak at $517 \mathrm{~nm}$. After stressing, the peak intensity is reduced to nearly half, whereas the spectral shape remains unchanged. Similar results have been obtained in devices stressed under $\mathrm{CW}$ and other pulsed currents. Figure 3.6(b) shows the micrographs of the unstressed and stressed OLEDs taken at $20 \mathrm{~mA} / \mathrm{cm}^{2}$. As seen, the former shows uniform light emission, whereas sporadic dark spots start to emerge in the latter. We have found similar evolution of dark spots in unstressed OLEDs fabricated in the same run and stored in a glovebox. These observations thus confirm the previous finding that dark spots are not current-induced, but 
mainly result from reactions with atmosphere [109], especially in the presence of residual water incorporated during thin film deposition and released from the packaging materials. This type of defect, however, was found to cause little degradation of the OLED characteristics over the time frame of our current stressing tests.

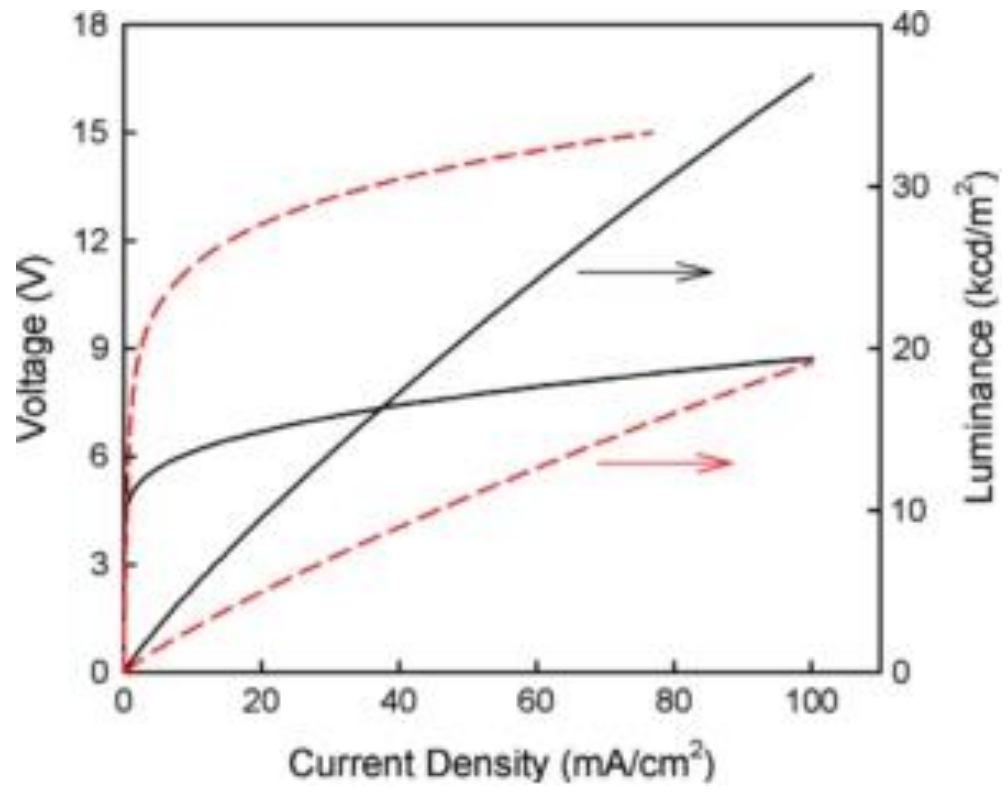

Figure 3.7 L-J-V characteristics of a phosphorescent OLED before (solid lines) and after (dashed lines) stressing under $50 \mathrm{~mA} / \mathrm{cm}^{2}$ pulsed current $(1 \mathrm{kHz}, 20 \%$ duty cycle) for $60 \mathrm{~h}$.

Figure 3.7 compares the luminance-current density-voltage (L-J-V) characteristics of the same OLED before and after stressing for $60 \mathrm{~h}$. At $20 \mathrm{~mA} / \mathrm{cm}^{2}$, the unstressed OLED has an operational voltage of $6.6 \mathrm{~V}$ and brightness of $9448 \mathrm{~cd} / \mathrm{m}^{2}$. Its current efficiency and EQE are $47.2 \mathrm{~cd} / \mathrm{A}$ and $12.7 \%$, respectively. After stressing with the current density of $50 \mathrm{~mA} / \mathrm{cm}^{2}$, its luminance reached $1.5 \times 10^{4} \mathrm{~cd} / \mathrm{m}^{2}$. The current stressing resulted in obvious performance degradation. At $20 \mathrm{~mA} / \mathrm{cm}^{2}$, the voltage increased by $5.6 \mathrm{~V}$ and the luminance decreased by $48 \%$. The optical decay of the encapsulated OLED can be largely attributed to intrinsic degradation mechanisms. A generally accepted intrinsic process responsible for luminescent loss in similar phosphorescent OLEDs is that electrochemical decomposition of organic materials in the EML under electrical excitation leads to the formation of defect states, which have been detected by 
chromatographic analysis and time-resolved photoluminescence [110-111]. These defects may act as charge traps, nonradiative recombination centers and luminescent quenchers. Earlier studies have revealed that the electrochemical reactions are mainly driven by exciton-polaron annihilation [112] and/or ionic species from reduction of residual water [110]. These reactions may be enhanced by significant self-heating as the majority of the electrical input power in the OLEDs is converted into heat rather than light. OLEDs have been found to exhibit a shorter lifetime at elevated temperatures presumably due to thermally assisted impurity diffusion and bond cleavage [113-114]. This is more pronounced in devices comprising materials with a low glass transition temperature. Since the OLEDs studied herein do not have an additional hole injection layer, there is a large energy barrier of $0.5 \mathrm{eV}$ for hole injection due to energy level misalignment between ITO and NPB. An additional voltage dropping at the ITO/NPB interface may produce a considerable amount of joule heat, accelerating the chemical transformation of amorphous NPB [115]. Defect states generated in this process may also act as carrier traps and recombination centers, leading to degraded performance.

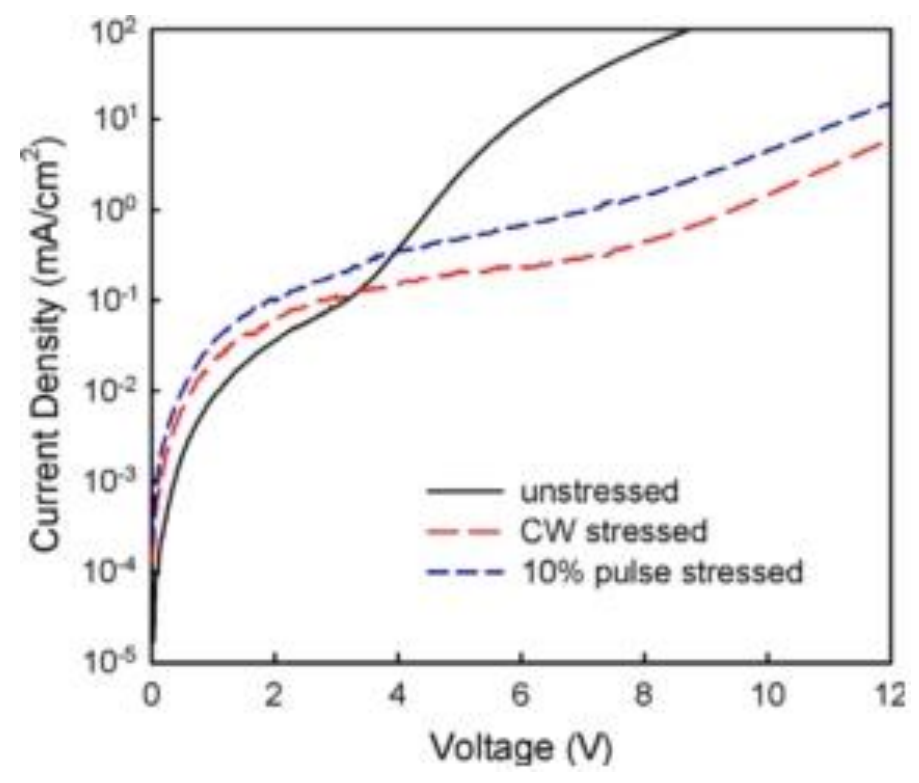

Figure 3.8 Forward J-V characteristics of phosphorescent OLEDs before and after stressing under 50 $\mathrm{mA} / \mathrm{cm}^{2} \mathrm{CW}$ current for $22 \mathrm{~h}$ or pulsed current $(1 \mathrm{kHz}, 10 \%$ duty cycle) for $89 \mathrm{~h}$.

The electrical degradation of the OLEDs is more clearly illuminated in Figure 3.8, a 
semi-log plot of the J-V characteristics of an unstressed OLED and OLEDs stressed under CW current for $22 \mathrm{~h}$ and $10 \%$ pulsed current for $89 \mathrm{~h}$. The respective luminance values of the two stressed OLEDs dropped to $39 \%$ and $59 \%$ of their initial values. As seen, the low-bias $(<3.5 \mathrm{~V})$ leakage current increases in both stressed OLEDs by several times as compared to that in the unstressed device. This suggests that some generated defects in the EML and charge transport layers function as shunt leakage paths. The fact the pulse-stressed device shows less optical loss but higher leakage current suggests that different types of defects may be responsible for the changes in the luminescence and leakage. In the high injection regime, the J-V curves show significant increases in the series resistance and operational voltage. At $20 \mathrm{~mA} / \mathrm{cm}^{2}$, the CWstressed OLED has a voltage 1.5 V higher than that of the pulse-stressed OLED, correlating well with a bigger luminance loss it suffered. The voltage increase in the stressed OLEDs is indicative of the presence of space charge limited current, which is limited by fixed charge filling up the deep traps generated during the stressing process [116].

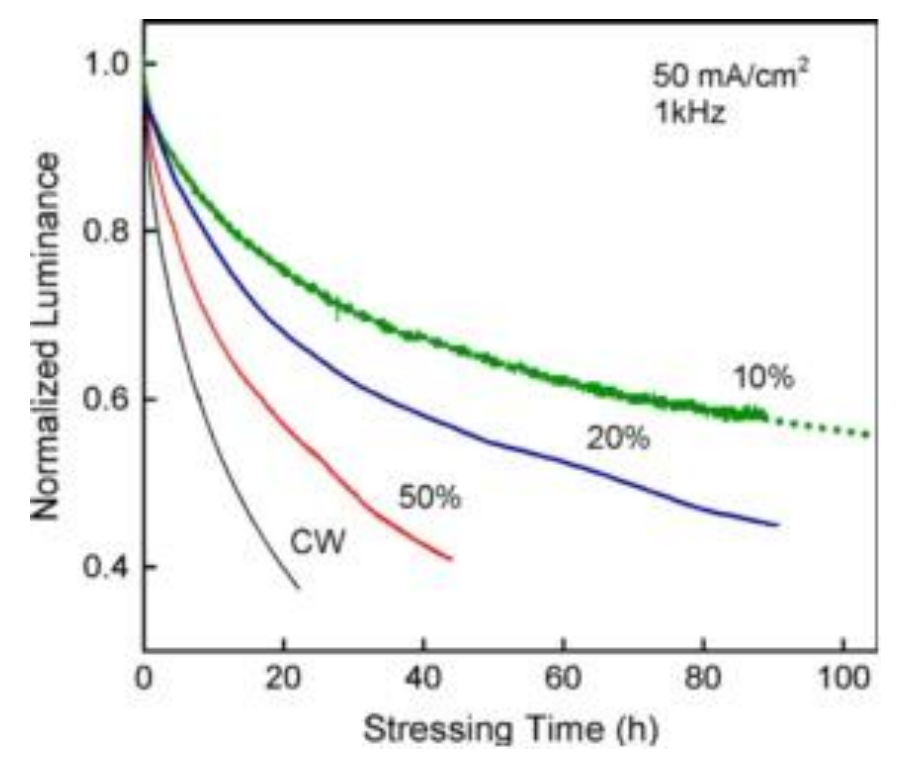

Figure 3.9 Evolution of the normalized luminance of phosphorescent OLEDs stressed under $50 \mathrm{~mA} / \mathrm{cm}^{2} \mathrm{CW}$ or $1 \mathrm{kHz}$ pulsed currents. The pulse duty cycle varies from $10 \%$ to $50 \%$.

Figure 3.9 shows the evolution of the normalized luminance of OLEDs stressed under different conditions. In all cases, the OLEDs exhibit typical stretched exponential decay, which 
appears to comprise two regimes: the initial rapid decay and subsequent slow decay. The $80 \%$ and $50 \%$ lifetimes of these OLEDs, which are defined as the times for the brightness to decline to $80 \%$ and $50 \%$ of their initial values, and denoted as $\mathrm{t}_{0.8}$ and $\mathrm{t}_{0.5}$, respectively, are summarized in Table 3.1. Under CW operation, $\mathrm{t}_{0.5}$ is $12.7 \mathrm{~h}$. The half life of the OLED at a lower brightness level can be estimated using the following equation:

$$
t_{0.5} \times L^{n}=\text { Constant }
$$

Table 3.1 t 0.8 and t to.5 of OLEDs stressed under $50 \mathrm{~mA} / \mathrm{cm}^{2} \mathrm{CW}$ current or $1 \mathrm{kHz}$ pulsed currents with different duty cycles.

\begin{tabular}{|c|c|c|c|c|}
\hline & CW & $50 \%$ duty cycle & $20 \%$ duty cycle & $10 \%$ duty cycle \\
\hline $\mathrm{t}_{0.8}$ & 2.0 & 4.3 & 8.7 & 13.7 \\
\hline Effective $\mathrm{t}_{0.8}$ & 2.0 & 2.15 & 1.74 & 1.37 \\
\hline $\mathrm{t}_{0.5}$ & 12.7 & 28.6 & 71.1 & 145.8 \\
\hline Effective $\mathrm{t}_{0.5}$ & 12.7 & 14.3 & 14.22 & 14.58 \\
\hline
\end{tabular}

where $\mathrm{L}$ is the brightness and $\mathrm{n}$ is the acceleration factor. Using $\mathrm{n}=1.9$ [117], we can find that $\mathrm{t}_{0.5}$ at $300 \mathrm{~cd} / \mathrm{m}^{2}$ is $2.1 \times 10^{4} \mathrm{~h}$. As seen in Table 3.1 , the effective $80 \%$ lifetimes, defined as t0.8 duty cycle, of the OLEDs stressed under pulsed currents with $20 \%$ and $10 \%$ duty cycles are $14 \%$ and $32 \%$ shorter than $\mathrm{t}_{0.8}$ of the CW-stressed OLED, respectively. In contrast, the effective $50 \%$ lifetimes of the pulse stressed OLEDs are found to be $12-15 \%$ longer than $\mathrm{t}_{0.5}$ of the $\mathrm{CW}$ stressed OLED. This discrepancy suggests that the luminance decay in the fast and slow decay regimes is governed by different mechanisms. It is plausible that processes independent of current excitation, like diffusion of metallic ions and environmental contaminants, play a nonnegligible role in the initial rapid decay behavior which determines $\mathrm{t}_{0.8}$, whereas current-induced thermal and nonthermal effects become dominant only in the subsequent slow decay regime which is better represented by $\mathrm{t}_{0.5}$. The small improvement (by 15\%) in the effective $50 \%$ lifetime under $10 \%$ pulsed stressing over $\mathrm{CW}$ operation can be partly attributed to the fact the real impact of pulsed current is smaller than $\mathrm{CW}$ current because the accumulated ions and 
carriers have chance to redistribute during the off cycles. All these results suggest that selfheating plays a small role in the current-induced electrochemical degradation process. In other words, pulsed operation does not remarkably extend the operational lifetime of the OLEDs compared to $\mathrm{CW}$ operation even at high injection levels. In the $\mathrm{CW}$ mode, significant localized heating may occur at the ITO/NPB interface, but our finding indicates that this has a small influence on the OLED stability.

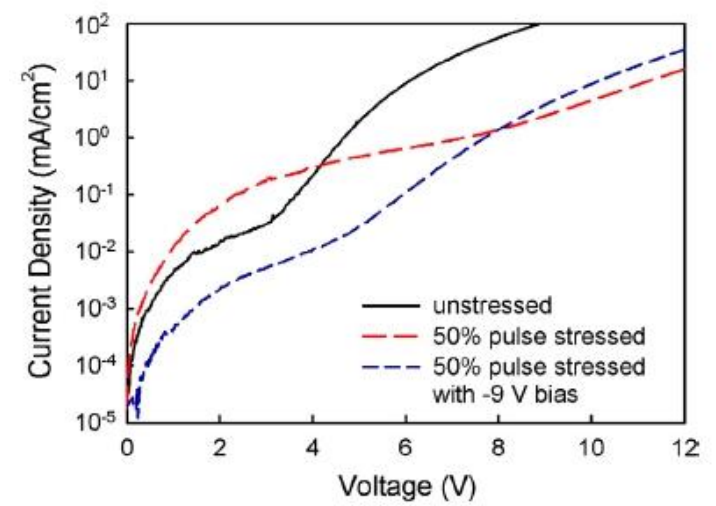

(a)

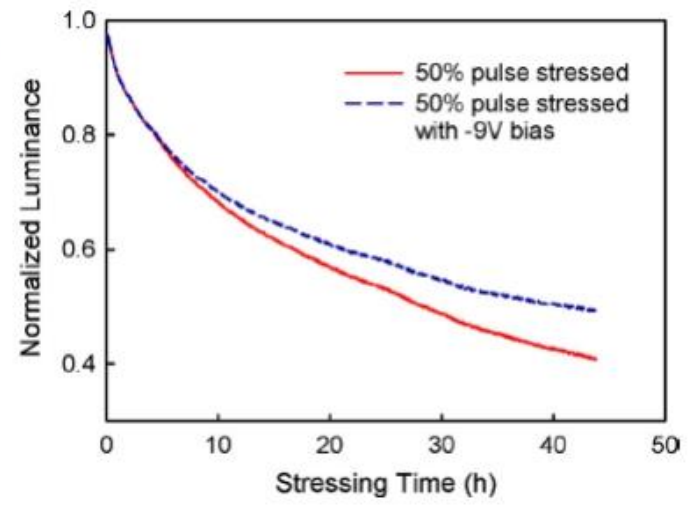

(b)

Figure 3.10 (a) Forward J-V characteristics of phosphorescent OLEDs before and after pulsed stressing at 50 $\mathrm{mA} / \mathrm{cm}^{2}(1 \mathrm{kHz}, 50 \%$ duty cycle) with and without a $-9 \mathrm{~V}$ bias. (b) Evolution of the normalized luminance of the phosphorescent OLEDs during $50 \mathrm{~mA} / \mathrm{cm}^{2}$ pulsed stressing with and without a $-9 \mathrm{~V}$ bias.

It has been found that the reliability of OLEDs can be improved using the ac driving scheme, where application of a proper reverse bias can induce partial performance recovery, leading to overall alleviated device degradation [118-119]. To validate this behavior, we applied a $-9 \mathrm{~V}$ bias to the OLEDs during the reverse cycle of the pulsed stressing, while keeping the current density in the forward cycle at $50 \mathrm{~mA} / \mathrm{cm}^{2}$. Figure 10 (a) compares the $\mathrm{J}-\mathrm{V}$ characteristics of the OLEDs stressed under 50\% pulsed currents for $44 \mathrm{~h}$ with and without a reverse bias. In contrast to the regular pulse stressing which led to an increase in the low-bias leakage current, pulsed stressing with a reverse bias considerably reduced the leakage. This may be explained by the fact that the reverse current is more localized and can burn out some defects which act as conducting micro- filaments in the OLED structure. At high bias, the voltage increase is also 
smaller in the device pulse-stressed with a reverse bias. Figure 3.10(b) shows the luminance decay behaviors of the two OLEDs. With an applied reverse bias, the half life (i.e., to.5) is increased by $47 \%$, from $28.6 \mathrm{~h}$ to $42 \mathrm{~h}$. The data verifies that adding a reverse component in the pulsed current can indeed cause performance recovery and thus slow down the device degradation.

The recovery effect, which has been found to be more pronounced with increasing reverse bias [120], can be attributed to two major reasons: (i)The reverse current removes some defects acting as conducting micro-channels, as discussed above. The same defects may also cause luminance loss; and (ii)The reverse bias can de-trap space charge from defect states and alleviates the accumulation of trapped charge caused by the injection current in the forward cycle [121]. Interestingly, the luminance decay in these two OLEDs is similar in the initial fast decay stage, and the reverse bias only suppresses luminance loss in the subsequent slow decay regime. This finding confirms our hypothesis that the initial fast decay is in part attributed to non-current factors, whereas the slow decay is predominantly caused by current-induced charge accumulation and defect generation.
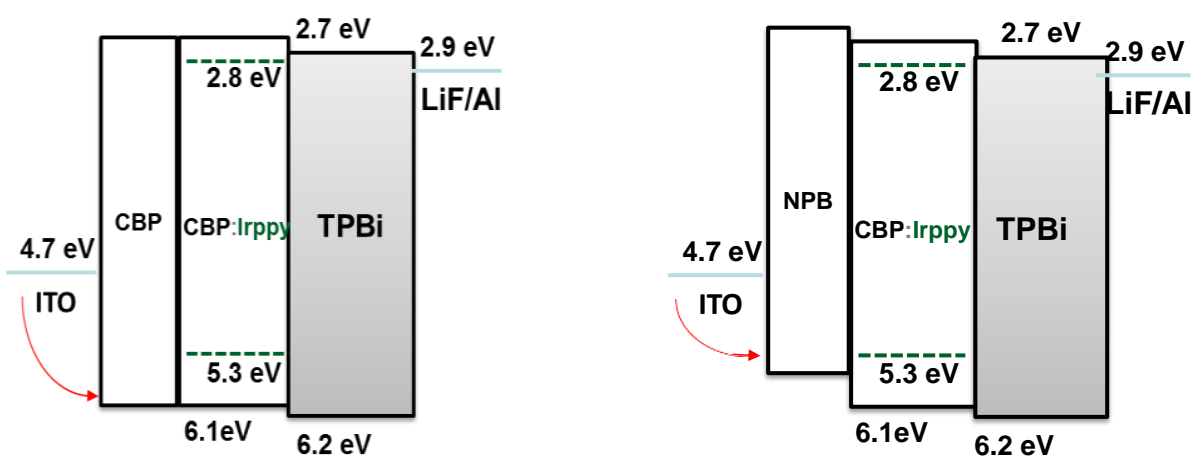

Figure 3.11 Band diagram of PhOLEDs with CBP HTL(left) and NPB HTL(right)

Then, more uniformed PhOLEDs with $40 \mathrm{~nm}$ CBP, same material as host in EML, replacing NPB as HTL were fabricated to further evaluate the role of joule heating during the OLEDs degradation process. The difference between these two structures in energy band diagram 
are shown in Figure 3.11 The HOMO of NPB is $5.4 \mathrm{eV}$, compared to $6.1 \mathrm{eV}$ for CBP. Therefore, the energy barrier for hole injection is much higher in the OLEDs with a CBP HTL.

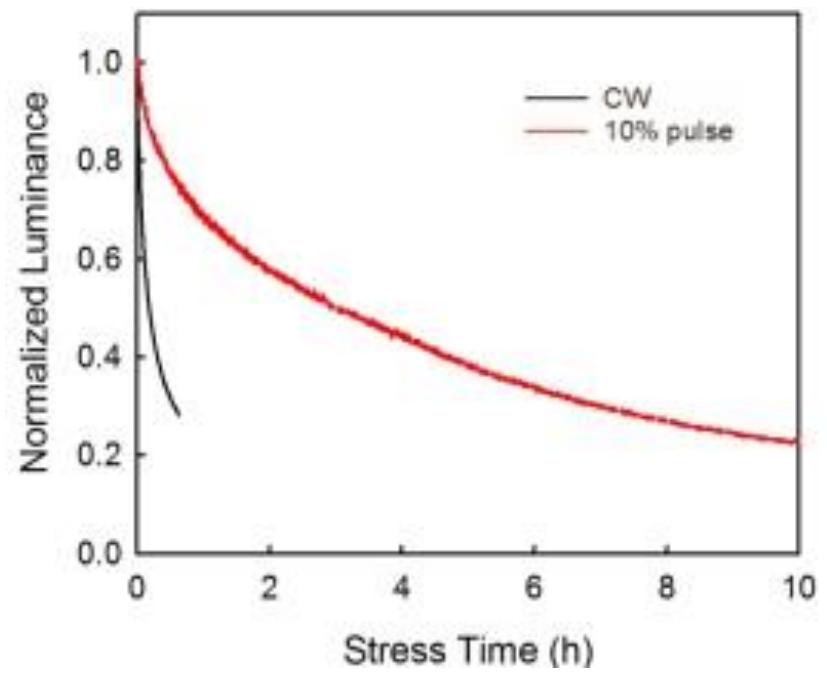

Figure 3.12 Evolution of the normalized luminance of PhOLEDs with CBP HTL stressed CW and $10 \%$ pulsed current

The devices are thus expected to suffer more from joule heating. Similarly, the devices with CBP as HTL were stressed under $\mathrm{CW}$ and pulse current stressing. The evolution of normalized luminance is shown in Figure 3.12. The effective half life of the OLEDs, is found to be $0.31 \mathrm{~h}$ under pulsed stressing, which is $70 \%$ longer than $\mathrm{t}_{0.5}$ under $\mathrm{CW}$ stressing $(0.18 \mathrm{~h})$. Therefore, all the results suggest that, unlike the case in OLEDs with a CBP HTL, joule heating plays a small role in the degradation process of the OLEDs with a NPB HTL, whose HOMO level is aligned reasonably well with the Fermi level in ITO. This finding confirms that voltage drop at the ITO/HTL interface and the resulting localized heating are an important factor driving intrinsic degradation of OLEDs.

\subsection{Conclusions}

In summary, performance degradation of green PhOLEDs with NPB as HTL under 50 $\mathrm{mA} / \mathrm{cm}^{2}$ pulsed current stressing was studied. The stressed devices exhibited increased low-bias leakage and series resistance. The luminance evolution consisted of an initial rapid decay regime 
and a subsequent slow decay regime, which were governed by different degradation mechanisms. Adding a reverse bias component to the stressing pulse led to defect removal and alleviated charge accumulation, and thus suppressed the low-bias leakage and current-induced luminance decay. In PhOLEDs with CBP as HTL, with a relatively high interfacial energy barrier (1.4 eV), pulsed current stressing was conducted to suppress the joule-heating effect, leading to a $70 \%$ increase in the effective half life compared to CW stressing for PhOLEDs. In contrast, PhOLEDs with NPB as the HTL, with a relatively low interfacial energy barrier $(0.7 \mathrm{eV})$, only $\sim 15 \%$ improvement was obtained, indicating a minor heating effect. Thus, reducing the energy barrier between charge transport layers and the anode and reducing the overall operation voltage are essential for producing OLED devices with better reliability. In Chapters $4 \& 5$, we will try to achieve this goal by introducing inorganic p-type dopants into the organic hole transport layer which not only enhances the electrical conductivity but also effectively reduces the interfacial barrier for hole injection. 


\section{Chapter 4 P-type Doping of Hole Transport Materials with Inorganic Transition Metal Oxides in PhOLEDs}

\subsection{Introduction}

State-of-the-art phosphorescent OLEDs (PhOLEDs) typically have a complicated multilayer device structure comprising charge injection, transport, and blocking layers surrounding the emitting layer (EML). These layers must be carefully designed and deposited to enable balanced electron and hole current flows as well as effective confinement of excitons in the EML. From the manufacturing perspective, the multilayer architecture is not cost competitive as it complicates the fabrication procedure. More importantly, charge accumulation, exciton quenching, electric field buildup, and joule heating at heterointerfaces can be important factors limiting the device efficiency and lifetime. Based on the conclusions in Chapter 3, a better energy alignment at interfaces is needed to suppress joule heating which may cause accelerated aging of OLEDs.

One way to simplify the PhOLED structure is by electrical doping, which has been developed to enhance the charge transport capability of organic materials, and led to highefficiency PhOLEDs based on a simple p-i-n structure [122]. Introducing organic or inorganic dopants in the organic charge transport layers can dramatically increase the free carrier density by several orders of magnitude and thus improve their electrical conductivity, giving rise to a

reduced operation voltage of PhOLEDs [123-125]. Doping can also remarkably reduce the interfacial energy barriers between the electrodes and charge transport layers [123], and therefore eliminate the need for additional charge injection layers.

Another strategy to simplify the PhOLED structure is to use an ambipolar organic semiconductor as the host material in the EML as well as the charge transport material [126]. For 
example, high-efficiency green PhOLEDs can be simplified on top of plasma-chlorinated indium-tin-oxide (ITO)/glass substrates, where ambipolar CBP was employed as the material for the EML host as well as hole transport layer (HTL) [127-128].

Yun et al. successfully developed homojunction p-i-n PhOLEDs based on a single organic semiconductor with performance comparable to that of conventional complex multilayer PhOLEDs. $\mathrm{MoO}_{3}$ doped CBP, $\operatorname{Ir}(\text { ppy })_{2}$ (acac) doped CBP and Caesium doped CBP was used as the HTL, EML and ETL separately [129]. These simplified PhOLEDs enjoy the advantages of

easy fabrication. Inspired by their work, more dopants in organic CBP need to be further explored to realize more efficient and more reliable operation by optimizing the doping concentration of each doped layer to achieve a better current balance condition.

In this chapter, a systematic study of doping behavior of wide bandgap ambipolar CBP with different transition metal oxides (TMOs) are reported through detailed electrical device characterization. First, the doping mechanisms in organic semiconductors are discussed and the use of TMOs as p-type dopants in organic electronic devices is reviewed. Secondly, the doping of $\mathrm{CBP}$ with $\mathrm{WO}_{3}$ is fully investigated. Hole-only devices with a $\mathrm{WO}_{3}$ doped or undoped CBP HTL are fabricated and characterized to optimize the doping level and thickness. Then, simplified PhOLEDs with a $\mathrm{WO}_{3}$-doped CBP HTL and an $\operatorname{Ir}(\mathrm{ppy})_{3}$ doped CBP EML are fabricated. Thirdly, doping of $\mathrm{CBP}$ with another $\mathrm{TMO}, \mathrm{MoO}_{3}$ is also studied by characterization of hole-only devices with a $\mathrm{MoO}_{3}$ doped CBP HTL. Finally, doping of CBP with non-transition-metal oxides such as $\mathrm{ZnO}$ and $\mathrm{SnO}_{2}$ is also conducted in order to validate the doping mechanism with TMOs.

\subsection{Doping of Organic Semiconductors with TMOs}

The basic principle of doping in organic semiconductors is that mobile carriers from an electron donor (n-type) or acceptor (p-type) are injected into the host semiconductor. Electrons are injected into the LUMO for n-type doping, whereas holes are injected into the HOMO for ptype doping. As mentioned previously, organic semiconductors have low intrinsic charge carrier 
concentration, and the mobility in an intrinsic amorphous organic semiconductor is very low. Thus, a significant amount of energy must be used to transport charged carriers within organic layers, which is manifested as a relative large voltage drop in typical organic devices [130].

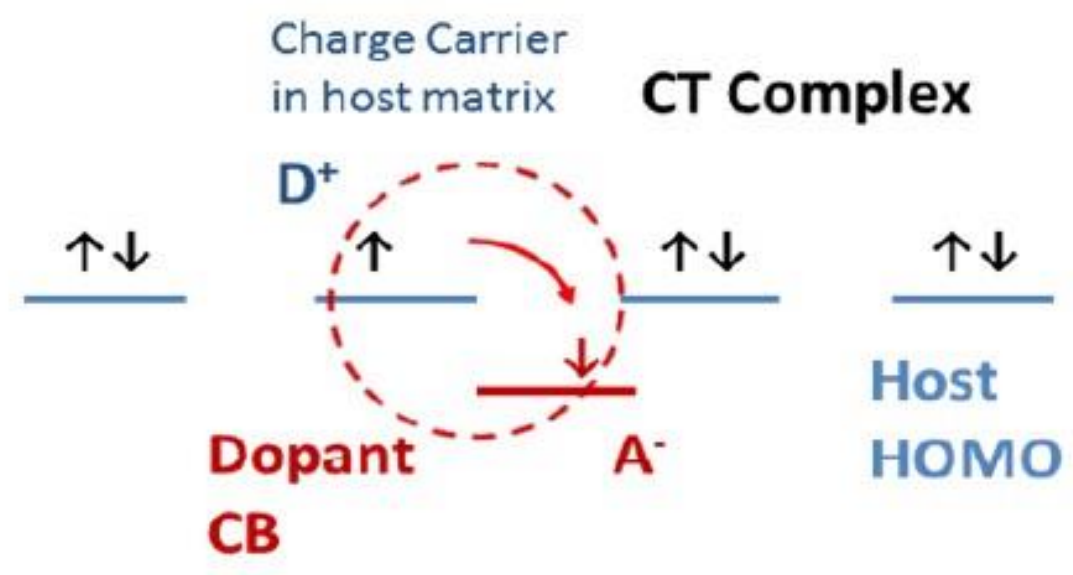

Figure 4.1 Charge transfer complex formation in p-type doping system [130]

For effective p-type doping as shown in Figure 4.1, a dopant material with electron accepting characteristics is needed. In order to allow for effective transfer and transport, electrons from the filled HOMO must be able to charge transfer into the unfilled dopant levels, thus forming mobile holes in the HOMO of the host matrix. This would dictate that the dopant material would have a Conduction Band (CB) energy level lower than the HOMO level of the organic semiconductor [131]. In essence, the transfer of electrons from the HOMO of the host matrix into the dopant CB forms a Charge Transfer (CT) complex. With the aid of the CT complex, charges have a faster alternate transport path compared to the slow intrinsic hopping process [132]. The doped transport layer exhibits significantly higher mobility and provides an effective means to transport charge carriers without a large voltage drop across the transport layer showing that the primary features of doping in an organic semiconductor are increased conductivity, and a shift in the effective work function of the HTL. These correspond to improvement in transport and injection for the doped HTL.

In short-wavelength PhOLEDs emitting green and blue light, the host material in the 
EML typically has a wide bandgap. For efficient charge injection into the EML, the charge transport materials should also have a wide bandgap and thus a deep-lying HOMO level. For example, CBP, with a relatively high hole mobility $\left(\sim 10^{-3} \mathrm{~cm}^{2} / \mathrm{V} \cdot \mathrm{s}\right)$ compared to typical hole transport materials like $\alpha$-NPD $\left(\sim 10^{-4} \mathrm{~cm}^{2} / \mathrm{V} \cdot \mathrm{s}\right)$ [133] and spiro-TAD $\left(\sim 10^{-4} \mathrm{~cm}^{2} / \mathrm{V} \cdot \mathrm{s}\right)$ [134], is used as the host material as well as the hole transport material in some novel PhOLED architectures. The HOMO level of CBP was found by UPS to be $6.1 \mathrm{eV}$ and its band gap is 3.1 $\mathrm{eV}$ [135-136]. As discussed in previous Section 3.3, The deep HOMO level of CBP allows holes to freely flow into the EML and prevents charge accumulation at the CBP/EML interface. Such accumulation which is prevalent in traditional designs has been found to significantly reduce device performance [137]. However, this also result in a large barrier for hole injection from the ITO anode. The energy barrier between CBP and ITO would be $>1 \mathrm{eV}$, hampering hole injection in LEDs with a CBP HTL [138]. Doping can greatly mitigate the issue and enhance charge transport within CBP as well, but the choices for appropriate p-type dopants are very limited due to its wide bandgap.

TMOs have been investigated for decades, but their introduction in the field of organic electronics dates back to the late 1990's, when Tokito reported a significant increase in holeinjection when using thin films of vanadium, molybdenum and ruthenium oxides as interlayers between the anode and the organic material in organic light emitting diodes (OLED) [139]. This initial work was followed by a series of reports on the use of such compounds in OLEDs and organic photovoltaic (OPV) devices, as hole-injection and hole-extraction interlayers, and in charge generation and charge recombination layers [140]. Interest in TMO films stemmed predominantly from their reported high work function, their semiconducting properties and their good transparency, characteristics that are all very important for electrodes or for charge generation/ recombination materials.

Transition metals are elements with partially filled d orbitals, with one to nine electrons in the outer shell. In a solid, these d orbitals form relatively narrow d bands. For example, in the 
case of vanadium the next higher $4 \mathrm{~s}$ band is substantially broader and overlaps with the entire $\mathrm{d}$ band, resulting in an occupied $4 \mathrm{~s}$ band and metallic conductivity of the transition metal. In TMOs, the $2 p$ orbitals, which originate from the oxygen anion, are completely filled and form the valence band of the material. In the case of $\mathrm{V}_{2} \mathrm{O}_{5}$ the metallic cations form the $3 \mathrm{~d}$ band, which is partially filled. Because of the bonding/anti-bonding splitting between the $2 \mathrm{p}$ and $4 \mathrm{~s}$ bands, the cationic $4 \mathrm{~s}$ band is several eVs above the $3 \mathrm{~d}$ band and therefore completely empty at zero temperature. The cation $3 \mathrm{~d}$ band (conduction bands) is therefore responsible for the electronic and magnetic properties of the material. A wide range of applications, which are of particularly high technological importance, arise from those properties. Transition metals are used as functional components in catalysis, in high temperature superconductors, in magnetic recording devices based on the giant magnetoresistance effect [141], or in gas sensors [142-143] and electro-/photo-/thermos-chromic devices [144].
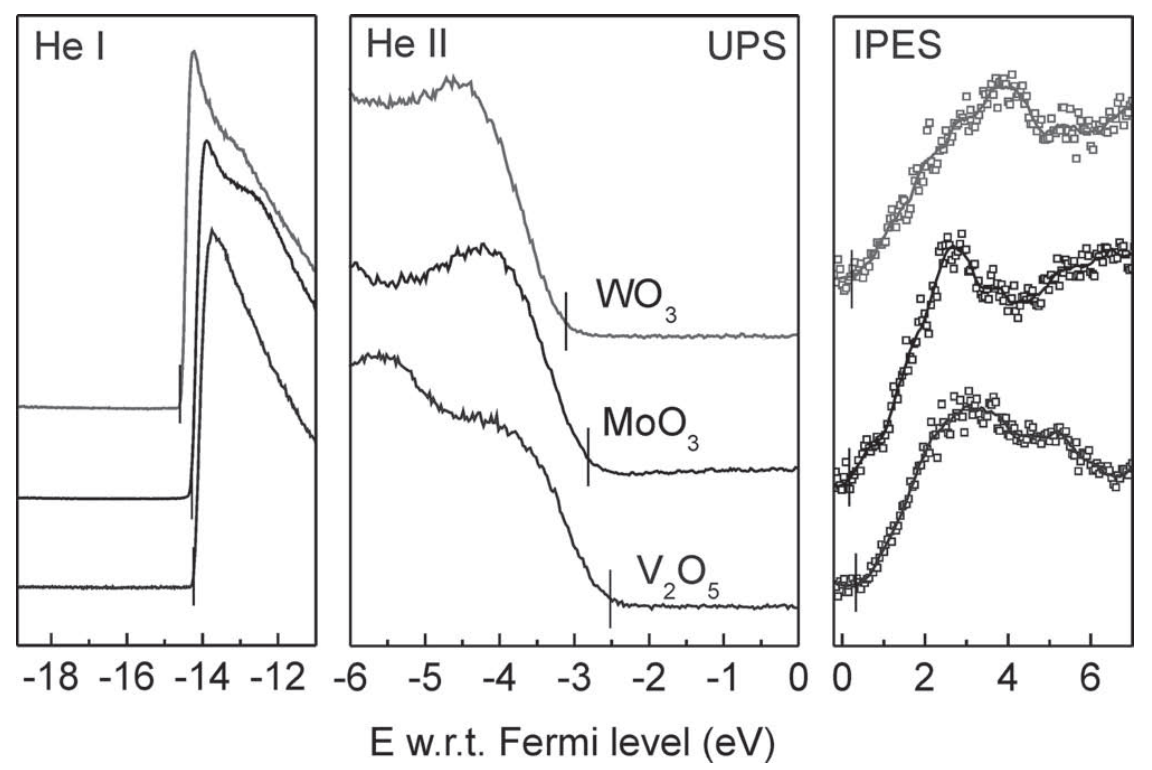

Figure 4.2 UPS and IPES spectra of vacuum grown $\mathrm{MoO}_{3}, \mathrm{~V}_{2} \mathrm{O}_{5}$ and $\mathrm{WO}_{3}$.

The left panel shows the photoemission onset, the middle panel shows the density of filled states near the VB edge, and the right panel shows the density of empty states near the CB edge. The reference is the Fermi level, measured separately on a metallic electrode. The tick marks denote the onset position, the top of the VB, and the bottom of the conduction band [144].

Figure 4.2 shows the combined UPS/IPES spectra taken from $\mathrm{MoO}_{3}, \mathrm{~V}_{2} \mathrm{O}_{5}$ and $\mathrm{WO}_{3}$ 
layers. The three panels include, from left to right: the photoemission onset, from which the vacuum level of the surface can be deduced; the density of states near the valence band edge; and the density of states near the conduction band edge.

Table 4.1 Ionized energy (IE), work function (WF) and electron affinity (EA) of $\mathrm{MoO}_{3}, \mathrm{~V}_{2} \mathrm{O}_{5}$ and $\mathrm{WO}_{3}$ measured via UPS and IPES. Determined by XAS/XES measurements from band gap [144].

\begin{tabular}{lccc}
\hline & $\mathrm{IE}$ & $\mathrm{WF}$ & $\mathrm{EA}$ \\
\hline $\mathrm{MoO}_{3}$ & $9.7 \mathrm{eV}$ & $6.9 \mathrm{eV}$ & $6.7 \mathrm{eV}$ \\
& $9.39 \mathrm{eV}$ & $6.58 \mathrm{eV}$ & $6.18 \mathrm{eV}$ \\
& $9.6 \mathrm{eV}$ & $6.86 \mathrm{eV}$ & $6.36 \mathrm{eV}$ \\
& $9.6 \mathrm{eV}$ & $6.9 \mathrm{eV}$ & $6.9 \mathrm{eV} *$ \\
& $9.15 \mathrm{eV}$ & & $6.35 \mathrm{eV}$ \\
$\mathrm{V}_{2} \mathrm{O}_{5}$ & $9.5 \mathrm{eV}$ & $7.0 \mathrm{eV}$ & $6.7 \mathrm{eV}$ \\
& $9.3 \mathrm{eV}$ & $6.8 \mathrm{eV}$ & \\
$\mathrm{WO}_{3}$ & $9.8 \mathrm{eV}$ & $6.7 \mathrm{eV}$ & $6.5 \mathrm{eV}$ \\
\hline
\end{tabular}

All spectra are plotted with the Fermi level as common reference. The work function (WF) of each TMO film can be directly determined from the photoemission onset via a very standard method, i.e., by translating the photoemission onset by the photon energy to obtain the vacuum level position $\left(\mathrm{E}_{\mathrm{vac}}\right)$ and by comparing this position with the Fermi level $\left(\mathrm{E}_{\mathrm{f}}\right)$ determined separately on a metallic electrode. The valence band spectrum of each film was measured with both He I and He II $(40.8 \mathrm{eV})$ radiations to assure that no component of the TMO VB edge resulting from parasitic photon lines was taken into consideration in the determination of the materials IE or EA. All the spectra clearly point out that $\mathrm{MoO}_{3}, \mathrm{~V}_{2} \mathrm{O}_{5}$ and $\mathrm{WO}_{3}$ exhibit very similar deep lying electronic states, with a VB edge around 2.5-3 eV below the Fermi level and a $\mathrm{CB}$ edge very close to the Fermi level, indicative of a highly n-type material. The n-type conductivity of these oxides is most likely a result of a slightly non-stoichiometric composition, with some oxygen deficiency, a situation which has been confirmed via X-ray photoemission 
spectroscopy (XPS) by several groups [145]. These TMOs are also often denoted as $\mathrm{MoO}_{\mathrm{x}}, \mathrm{V}_{2} \mathrm{O}_{\mathrm{x}}$ and $\mathrm{WO}_{\mathrm{x}}$ in the literature. Fully stoichiometric $\mathrm{MoO}_{3}, \mathrm{~V}_{2} \mathrm{O}_{5}$ and $\mathrm{WO}_{3}$ are known to be insulators. However, as commonly accepted in the literature, we denote materials as labeled by the supplier such as $\mathrm{MoO}_{3}, \mathrm{~V}_{2} \mathrm{O}_{5}$ and $\mathrm{WO}_{3}$ even though the film composition may be slightly nonstoichiometric after evaporation in vacuum.

The energetics of $\mathrm{MoO}_{3}, \mathrm{~V}_{2} \mathrm{O}_{5}$ and $\mathrm{WO}_{3}$ deduced from our PES/IPES studies are summarized in the energy diagrams of Figure 4.3. For comparison, Table 4.1 displays all energy level values from other groups. Results are in good agreement with those obtained for similarly vacuum-deposited $\mathrm{MoO}_{3}$ films by Subbiah et al. [146]and Kanai et al. [147]. The similarity between the three materials is impressive that $\mathrm{V}_{2} \mathrm{O}_{5}$ exhibits the largest $\mathrm{WF}(7.0 \mathrm{eV})$ closely followed by $\mathrm{MoO}_{3}$ and $\mathrm{WO}_{3}$ with values of $6.9 \mathrm{eV}$ and $6.7 \mathrm{eV}$, respectively.
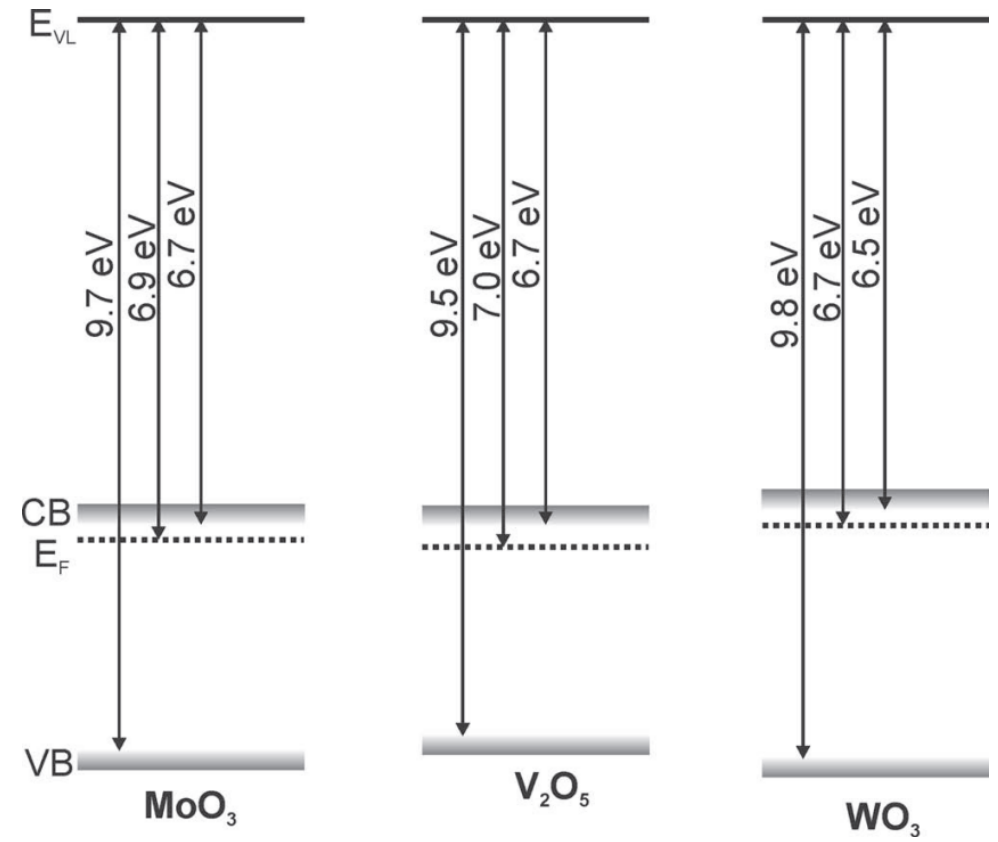

Figure 4.3 Electrical Band Structure for some Transition Metal Oxide (CB minimum and $\mathrm{VB}$ maximum of $\mathrm{MoO}_{3}, \mathrm{~V}_{2} \mathrm{O}_{5}, \mathrm{WO}_{3}$ ) [147]

The VB edge, or hole-transport level, of all three TMOs derives from the occupied O $2 p$ orbital. However, the unusually deep VB (ionization energy IE $>9 \mathrm{eV}$ ) makes hole-injection and transport highly unlikely in these materials. On the other hand, the strong n-type nature of these 
TMO films makes electron transport via the CB far more favorable. The TMO CB derives from the unoccupied transition metal d orbital. Aside from the p-type vs. n-type confusion stemming from the literature, discrepancies also appear in the range of different energy level values reported by various groups. Since TMOs have a large electron affinity above the HOMO of CBP, they are thus well suited for p-type doping. Recent efforts have led to the realization of effective $\mathrm{MoO}_{3}$ doping in CBP, NPB, TCTA [148-149]. The doping effect, as discussed in previous section, has been attributed to the formation of CT complexes through electrons transferring from organic host molecules to the inorganic dopants.

\subsection{Experimental Procedure}

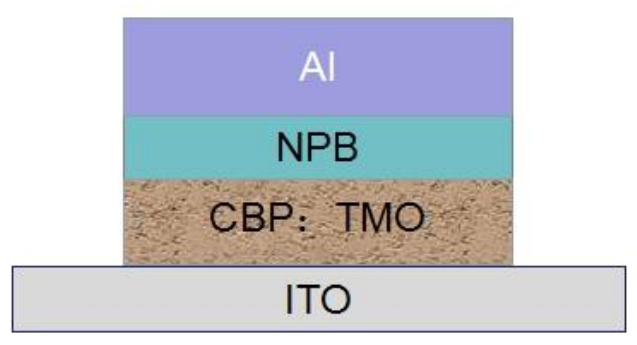

(a)

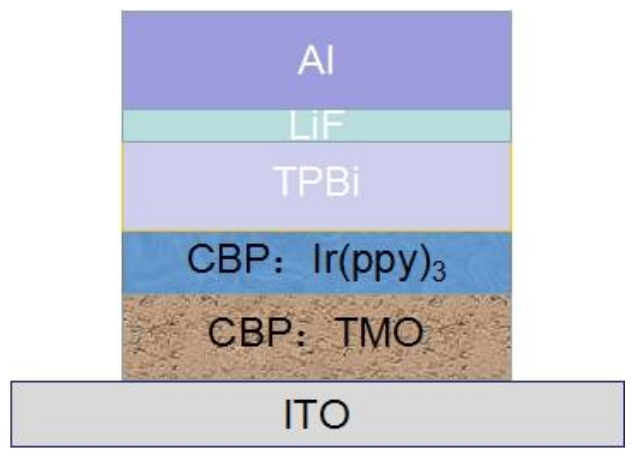

(b)

Figure 4.4 Schematic layer structure of (a) hole-only devices and (b) simplified PhOLED devices with TMO doped CBP as HTL

Green phosphorescent OLEDs and hole-only devices and were fabricated on glass substrates with pre-patterned ITO (sheet resistance $15 \Omega / \square$ ). The substrates were first cleaned with solvents and deionized water, and treated with $\mathrm{O}_{2}$ plasma for $5 \mathrm{~min}$. They were then transferred to a thermal evaporation system, where the organic layers and inorganic layers were deposited at a pre-calibrated rate $\sim 0.1-0.3 \mathrm{~nm} / \mathrm{s}$ and $\sim 0.005 \mathrm{~nm} / \mathrm{s}$. The OLED structure, as shown in Figure 4.4(b), consisted of a $40 \mathrm{~nm}$ CBP doped with $30 \mathrm{~mol}^{2} \mathrm{WO}_{3}$ or $\mathrm{MoO}_{3}$ as $\mathrm{HTL}$, a $30 \mathrm{~nm}$ CBP doped with 7 wt.\% fac-tris(2-phenylpyridinato-N,C2') iridium (III) [Ir(ppy) 3 ] light-emitting 
layer, and a $45 \mathrm{~nm}$ TPBi electron transport layer. Finally, a $0.5 \mathrm{~nm} \mathrm{LiF/} 100 \mathrm{~nm}$ Al cathode was deposited through a shadow mask, which defined the active area of the OLEDs to be $0.1 \mathrm{~cm}^{2}$. Four identical OLEDs were fabricated on each substrate. They were encapsulated with epoxy and a glass lid in a $\mathrm{N}_{2}$-filled glovebox and characterized in air at room temperature.

As shown in Figure 4.4(a), hole-only devices consisted of pre-patterned ITO as anode, a $100 \mathrm{~nm}$ CBP doped with $0-40 \mathrm{~mol}_{\%} \mathrm{WO}_{3}, \mathrm{MoO}_{3}, \mathrm{ZnO}$ and $\mathrm{SnO}_{2}$ as hole transport layer, a $20 \mathrm{~nm}$ NPB as electron blocking layer and 100nm Aluminum as cathode. The holes are injected from ITO to HTL and transported by NPB and finally extracted from cathode. Because the $0.5 \mathrm{~nm} \mathrm{LiF}$ is not inserted between organic layer and aluminum, the work function of aluminum is $\sim 4.6 \mathrm{eV}$ which makes hard to inject electrons from cathode to organic layers. In addition, adding a $20 \mathrm{~nm}$ NPB as electron blocking layer will further prevent the electrons injections. Thus, the total current of hole-only device is mainly contributed from hole transport.

The current density-voltage (J-V) curves were measured using an Agilent $4156 \mathrm{C}$ semiconductor parameter analyzer and the current density was measured as a function of the applied voltage. The luminance of OLED devices was firstly measured by placing the device on top of a calibrated silicon photodiode and was swept with voltage from a Keithley sourcemeter. Then under a constant current-density $\left(50 \mathrm{~mA} / \mathrm{cm}^{2}\right)$ stressing, the luminance data was collected every 5 seconds to measure the lifetime of OLED devices.

\subsection{Results and Discussion}

\subsubsection{P-type doping of $\mathrm{CBP}$ with $\mathrm{WO}_{3}$}

$\mathrm{WO}_{3}$ has a deep-lying conduction band and a work function $\sim 6.7 \mathrm{eV}$. Compared with many other metal oxides, its evaporated films can retain a higher transparency due to limited reduction during thermal evaporation [150]. Therefore, it is expected to be an ideal p-type dopant in wide bandgap organic materials. Indeed, $\mathrm{WO}_{3}$ has successfully been used to dope NPB [151], 
TCTA [152], and 2-methyl-9,10-bis(naphthalen-2-yl) anthracene (MADN) [153], whereas its doping effectiveness in CBP, a common wide bandgap host material used in PhOLEDs, has not been investigated in detail.

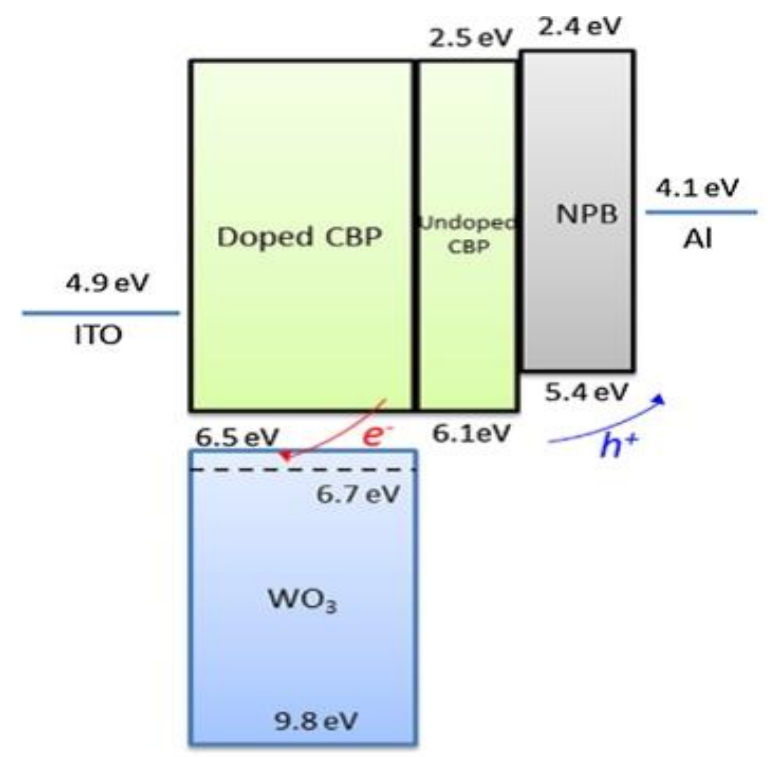

Figure 4.5 Energy diagram of hole-only device with a $\mathrm{WO}_{3}$-doped CBP HTL.

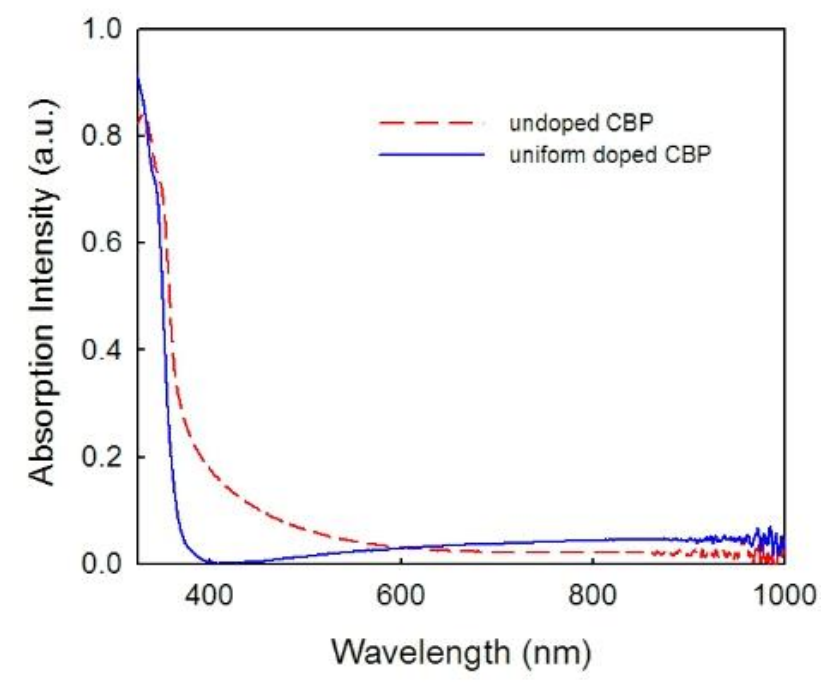

Figure 4.6 Absorption of thin film comprising $100 \mathrm{~nm} 30 \mathrm{~mol} \% \mathrm{WO}_{3}$ doped $\mathrm{CBP}$ and $100 \mathrm{~nm}$ undoped CBP

The work function of $\mathrm{WO}_{3}$ is greater than the ionization energy of $\mathrm{CBP}$. The $\mathrm{WO}_{3}$ dopants can thus accept electrons from the CBP host, generating free holes in CBP. Such a charge transfer process is illustrated in Figure 4.5. An increased absorption in doped CBP at long 
wavelengths beyond $750 \mathrm{~nm}$ was observed as compared to undoped CBP in Figure 4.6. Similar features have been seen in other metal oxide-doped organic materials and are often regarded as an indicator of the formation of charge transfer complexes [154-155].

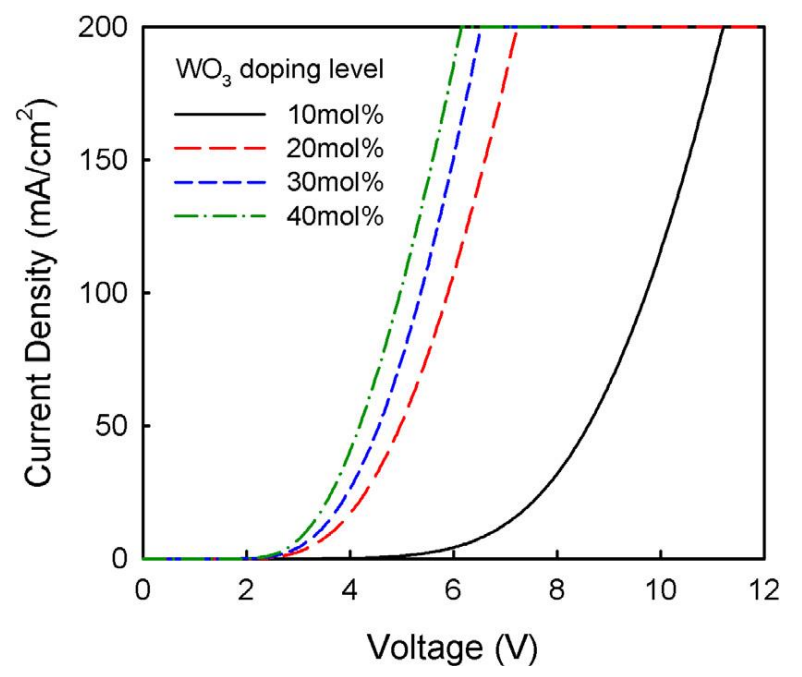

Figure 4.7 J-V characteristics of hole-only devices with a $40 \mathrm{~nm}$ 10-40mol\% WO3-doped/60 $\mathrm{nm}$ undoped CBP HTL.

In order to determine the optimal doping condition, hole-only devices with different $\mathrm{WO}_{3}$ doping levels and different doping thicknesses were fabricated and characterized. Figure 4.7 shows the $\mathrm{J}-\mathrm{V}$ curves of the devices comprising $40 \mathrm{~nm}$ doped CBP/60 $\mathrm{nm}$ undoped CBP, where the $40 \mathrm{~nm} \mathrm{CBP}$ was doped with 10-40mol\% $\mathrm{WO}_{3}$. For doping below $10 \mathrm{~mol} \%$, the deposition rates were hard to control during co-evaporation. Compared to the undoped device, the $10 \% \mathrm{WO}_{3}$-doped device has a slightly reduced forward voltage, whereas the device with $20 \%$ dopants exhibits a much smaller turn-on voltage. Above $20 \%$, the forward voltage continues to decrease with increasing doping level, though the slope of the decrease is much smaller, indicating saturation of the doping effect. These results suggest that there exists a critical doping concentration falling into the 10-20\% range, below which effective doping cannot be obtained.

This change was found to be accompanied by a rapid Fermi level shift toward the host HOMO edge. Doping saturation at high concentrations is believed to arise from the Fermi level pinning at the point where a large density of HOMO states exists [156-157]. Our finding that a 
high doping level of $\sim 20 \mathrm{~mol} \%$ is required to achieve effective doping is not surprising, as many metal oxide dopants in organic materials have a characteristic low doping efficiency [158]. In some cases, a doping level more than $50 \mathrm{~mol} \%$ is needed to achieve the maximum conductivity [159]. Nanoclusters of $\mathrm{MoO}_{3}$ have been observed in $\mathrm{MoO}_{3}$-doped NPB and CBP, explaining the low doping efficiency of a few percent as well as weak charge scattering effects at high concentrations up to 50wt\% [160]. Similar behaviors can be expected for $\mathrm{WO}_{3}$-doped CBP, as earlier studies have shown that thermally evaporated $\mathrm{WO}_{3}$ is in the form of $\left(\mathrm{WO}_{3}\right)_{3}$ nano-clusters [161]. Those trimmers may further agglomerate in the CBP matrix, resulting in a small host/dopant interfacial area which limits the doping efficiency. In the work described below, $30 \mathrm{~mol} \%$ doping was conducted in all doped structures.
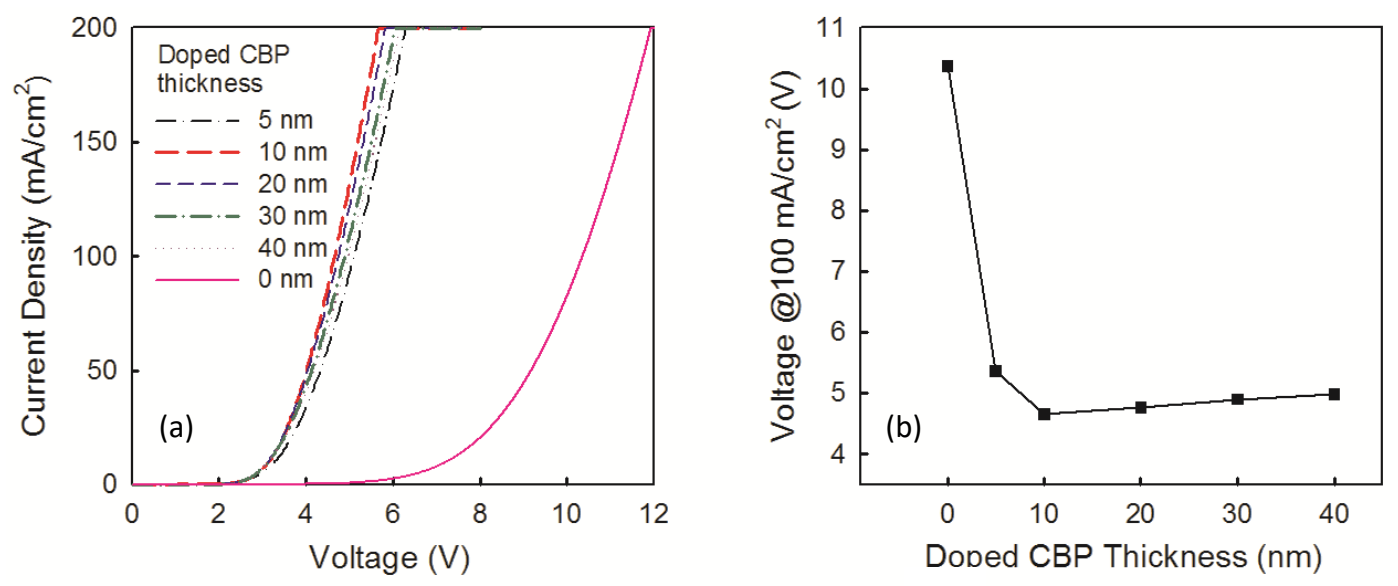

Figure 4.8 (a)J-V characteristics of hole-only devices with a $5-40 \mathrm{~nm} 30 \mathrm{~mol} \%$ WO3-doped/60 nm undoped CBP HTL. (b) The device voltage at $100 \mathrm{~mA} / \mathrm{cm}^{2}$ as a function of the doped CBP layer thickness.

For the purpose of efficient hole injection, the doping thickness should be greater than the width of the depletion region at the ITO/CBP interface. To quantitatively determine the critical doping thickness, we fabricated hole-only devices comprising 5-40 nm doped CBP/60 nm undoped CBP. Their J-V characteristics are shown in Figure 4.8(a). The forward voltage of the devices at $100 \mathrm{~mA} / \mathrm{cm}^{2}$ is plotted as a function of the doped layer thickness in Figure 4.8(b). With a $5 \mathrm{~nm}$ doped CBP, the device has a voltage of $5.4 \mathrm{~V}$. It drops sharply to $4.7 \mathrm{~V}$ for the device with $10 \mathrm{~nm}$ doped CBP. With a further increase in the doped layer thickness, the voltage 
increases gradually due to a non-negligible electric field dropped across the doped CBP layer. The data indicates that the depletion region width in $30 \mathrm{~mol}_{\%} \mathrm{WO}_{3}$-doped CBP is within $10 \mathrm{~nm}$.

An interesting observation from the above study is that the hole-only device with $40 \mathrm{~nm}$ doped CBP/60 nm undoped CBP had a much higher forward voltage than the device with 100 $\mathrm{nm}$ doped CBP. As seen in Figure 4.9, the difference at $100 \mathrm{~mA} / \mathrm{cm}^{2}$ is $1.4 \mathrm{~V}$, which seems to be too large to be solely attributed to the additional field dropping over the undoped layer. The enhanced conductivity in CBP is clearly seen in Figure 4.9, which compares the current densityvoltage (J-V) characteristics of hole-only devices with $100 \mathrm{~nm}$ undoped or $30 \mathrm{~mol} \% \mathrm{WO}_{3}$-doped CBP. With $\mathrm{WO}_{3}$ doping, the forward voltage at $100 \mathrm{~mA} / \mathrm{cm}^{2}$ decreases sharply from $10.4 \mathrm{~V}$ to $3.5 \mathrm{~V}$. In the undoped devices, the current is limited by slow hole transport as well as poor hole injection, as a large energy barrier is formed at the ITO/intrinsic CBP interface.

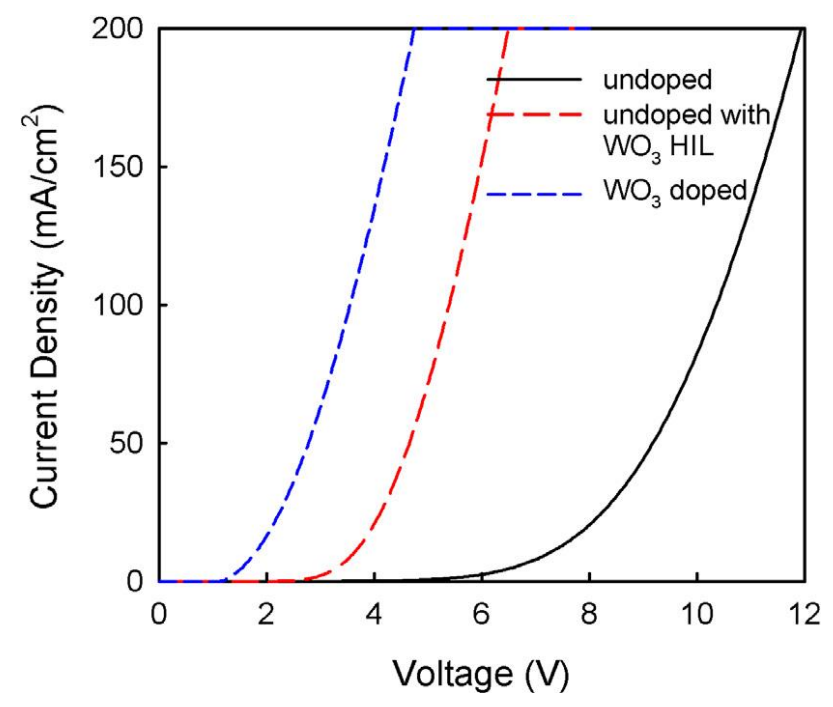

Figure 4.9 J-V characteristics of hole-only devices with a HTL comprising $100 \mathrm{~nm}$ undoped CBP, $100 \mathrm{~nm}$ 30mol\% WO3-doped CBP, or 0.5 nm WO $3 / 100$ nm undoped CBP.

As seen in Figure 4.9, the voltage of the doped device is also significantly smaller than that of an undoped device with a $0.5 \mathrm{~nm} \mathrm{WO} 3$ hole injection layer (HIL) $\left(5.4 \mathrm{~V}\right.$ at $\left.100 \mathrm{~mA} / \mathrm{cm}^{2}\right)$. These results suggest that $\mathrm{WO}_{3}$ doping improves not only the hole transport capability of CBP but also the efficiency of hole injection from the ITO anode. It also confirms that in OLEDs with a properly doped HTL, an additional HIL is not needed. The enhancement of hole injection into 
doped CBP can be explained as follows. Excess free holes generated from $\mathrm{WO}_{3}$ doping causes a downward shift of the Fermi level of CBP toward its HOMO edge, which have been confirmed by different methods including ultraviolet electron spectroscopy and Kevin probe [162-163]. As a result, a depleted CBP region forms near the ITO/CBP interface with a width decreasing with increasing doping concentration. This would facilitate hole tunneling, and effectively reduce the overall energy barrier for hole injection from the ITO anode into CBP.

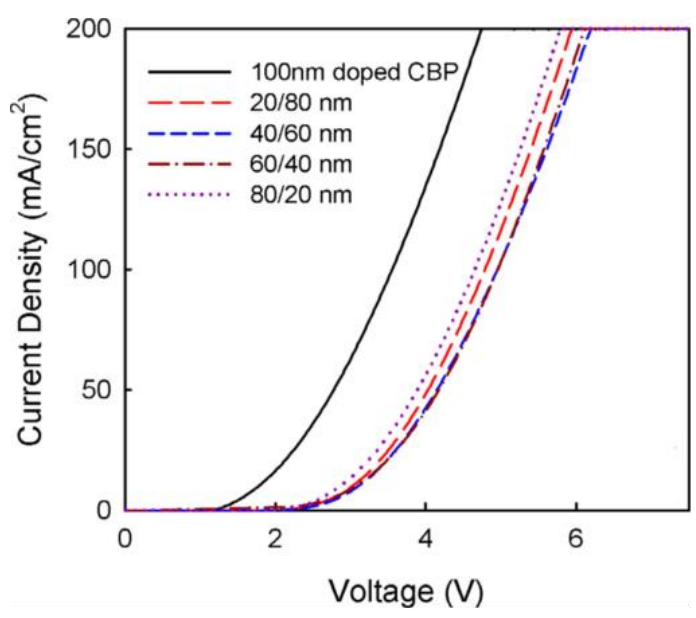

(a)

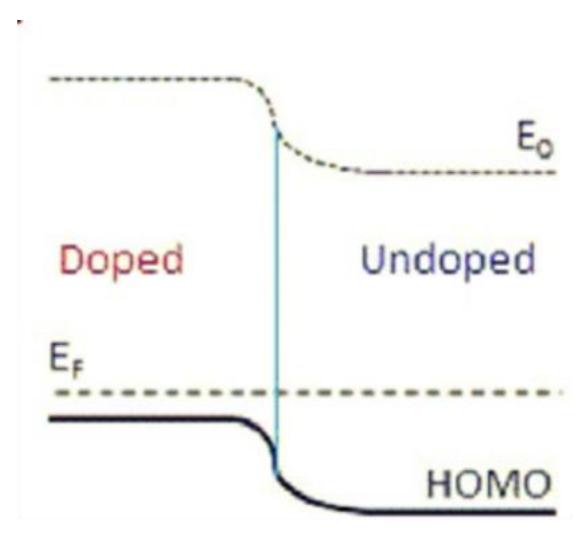

(b)

Figure 4.10 (a)J-V characteristics of hole-only devices with a $100 \mathrm{~nm}$ doped CBP HTL or an x/100-x nm doped/undoped CBP HTL and (b) the band diagram of a doped/undoped CBP junction.

To understand the origin of this behavior, we fabricated hole-only devices with 20/80, 40/60, 60/40, and 80/20 nm doped/undoped CBP layers. Their J-V curves are compared in Figure 4.10. As seen in Figure 4.10(a), they all have a similar voltage as the 40/60 device, suggesting that the $\sim 1.4 \mathrm{~V}$ additional bias arises from a voltage drop at the undoped/doped CBP interface. Considering the doping-induced changes in the energy level alignment, we can interpret this result as follows. As the Fermi level shifts toward the HOMO edge in the doped CBP, energy bands bend at the undoped/doped interface, upward on the doped side and downward on the undoped side. Note that the undoped CBP could be lightly doped near the interface due to impurity diffusion. An interfacial energy barrier is thus formed as illustrated in Figure 4.10(b), which must be overcome by an additional voltage drop. This effect is expected to be pronounced 
given a large amount of doping-induced Fermi level shift, which is likely the case of doped CBP.

Figure 4.11 compares the J-V characteristics of PhOLEDs with three different types of HTLs: $40 \mathrm{~nm}$ undoped CBP, $40 \mathrm{~nm} \mathrm{WO}_{3}$-doped CBP, and $30 \mathrm{~nm}$ doped/10 undoped CBP. The device with a $40 \mathrm{~nm}$ undoped CBP HTL has a high operation voltage of $10.8 \mathrm{~V}$ at $20 \mathrm{~mA} / \mathrm{cm}^{2}$, whereas the PhOLED with a $40 \mathrm{~nm}$ doped CBP HTL has a significantly lower voltage of $7.4 \mathrm{~V}$. As discussed before, this reduction arises from improved hole injection as well as hole transport in the doped CBP. Interestingly, the PhOLED with a $30 \mathrm{~nm}$ doped/10 $\mathrm{nm}$ doped HTL has a similarly low operation voltage despite an energy barrier existing at the doped/undoped interface as discussed earlier. On further thought, we realize that the same doped/undoped junction also exists in the PhOLED with $40 \mathrm{~nm}$ doped CBP because the CBP host in the EML is not p-type doped. This explains the comparable operation voltages in the two doped PhOLEDs.

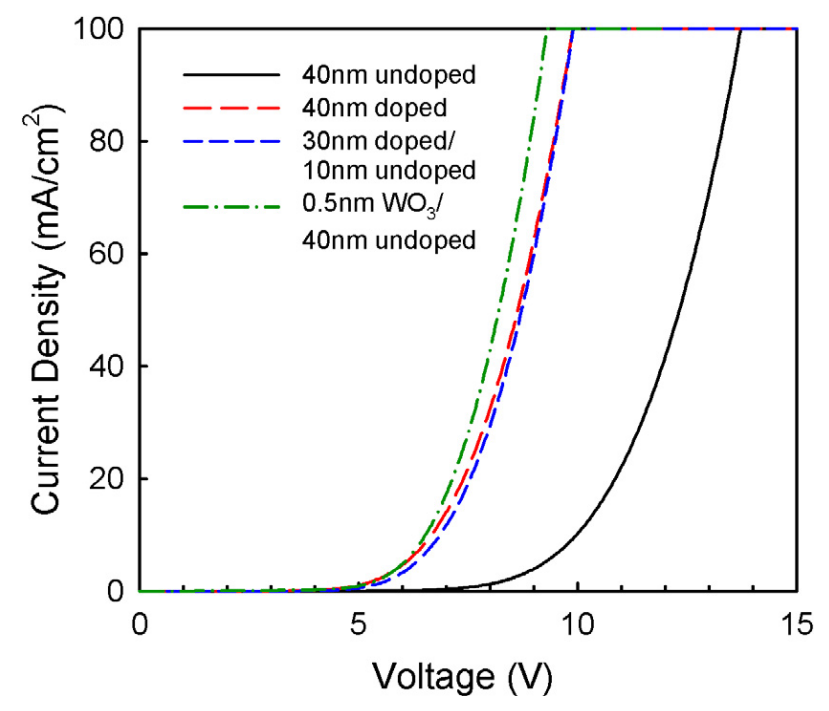

Figure 4.11 J-V characteristics of green PhOLEDs with different CBP HTLs.

Also, shown in Figure 4.12 is the J-V curve of an undoped PhOLED with a $0.5 \mathrm{~nm} \mathrm{WO}_{3}$ HIL, which exhibits a slightly smaller voltage than the doped devices. This result suggests that the extra voltage dropped over the $40 \mathrm{~nm}$ undoped CBP layer is not sufficient to overcome the barrier at the doped/undoped CBP interface. All the PhOLEDs exhibited typical green EL of $\operatorname{Ir}(\text { ppy) })_{3}$ with a peak at $517 \mathrm{~nm}$. Their luminance-current density (L-J) characteristics are 
compared in Figure 4.12(a). The undoped device is not efficient due to unbalanced hole and electron injection. With a $0.5 \mathrm{~nm} \mathrm{WO}_{3} \mathrm{HIL}$, hole injection from the ITO anode is markedly enhanced, giving rise to more balanced charge injection and thus more efficient luminescence. The luminance at $20 \mathrm{~mA} / \mathrm{cm}^{2}$ is raised from $2640 \mathrm{~cd} / \mathrm{m}^{2}$ sharply to $10613 \mathrm{~cd} / \mathrm{m}^{2}$. More balanced charge injection and transport is also achieved in the PhOLEDs having a doped CBP HTL without a HIL. At a given current density, the highest brightness is obtained in the PhOLED with a $30 \mathrm{~nm}$ doped/10 $\mathrm{nm}$ undoped CBP HTL, which is $~ 30 \%$ brighter than the PhOLED with $40 \mathrm{~nm}$ doped CBP. At $20 \mathrm{~mA} / \mathrm{cm}^{2}$, the brightness of the former is $11163 \mathrm{~cd} / \mathrm{m}^{2}$. The corresponding EQE is $13.6 \%$ and current efficiency is $55.8 \mathrm{~cd} / \mathrm{A}$. The comparison confirms that it is necessary to insert an undoped CBP layer between the doped HTL and EML to prevent impurity from diffusing into the EML and to suppress exciton quenching at $\mathrm{WO}_{3}$-related trapping states.
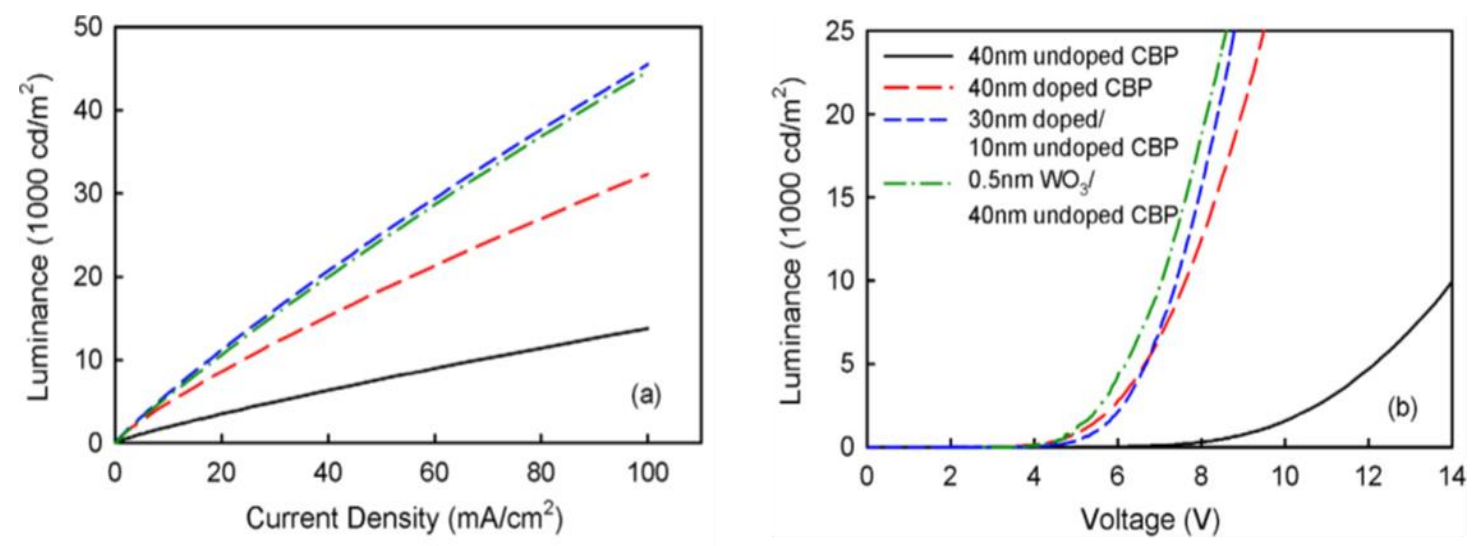

Figure 4.12 (a) L-J and (b) L-V characteristics of green PhOLEDs with different CBP HTLs

Figure 4.12(b) shows the luminance-voltage (L-V) characteristics of all four devices. The undoped device has a high luminescence turn-on voltage of $\sim 8 \mathrm{~V}$, whereas the other three devices turn on at $\sim 4 \mathrm{~V}$. At an operational voltage greater than $6.6 \mathrm{~V}$, the PhOLED with $30 \mathrm{~nm}$ doped/10 nm undoped CBP is brighter than that with $40 \mathrm{~nm}$ doped CBP. The brightness level of $10^{4} \mathrm{~cd} / \mathrm{m}^{2}$ is reached at $7.4 \mathrm{~V}$ and $7.6 \mathrm{~V}$ in these two devices, respectively. At this brightness, the former has a significantly lower input electrical power, and its power efficiency is $40 \%$ higher compared to the latter. The PhOLED with a thin $\mathrm{WO}_{3} \mathrm{HIL}$, which benefits from a lower current turn-on 
voltage as seen in Figure 4.12(b), actually has slightly higher brightness and power efficiency than the doped devices at a given operation voltage.

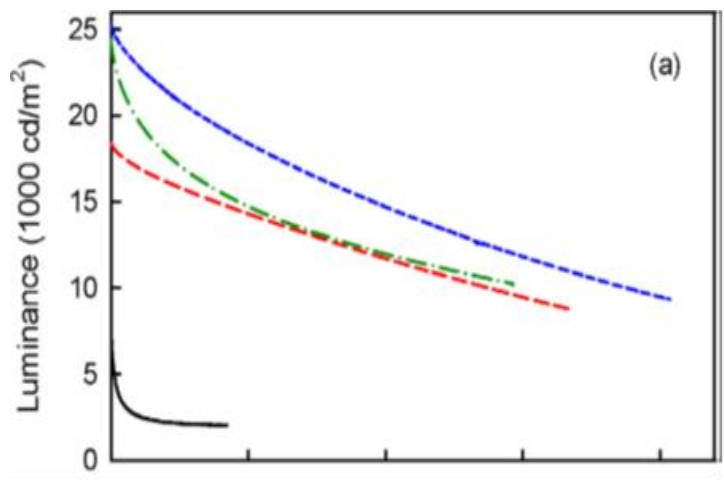

Stress Time (h)

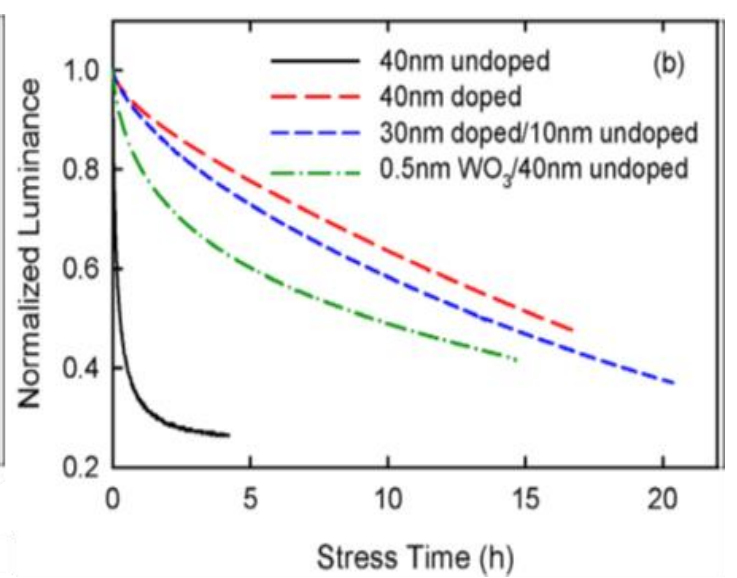

Stress Time (h)

Figure 4.13 Evolution of the raw (a) and normalized (b) luminance of green PhOLEDs with different CBP HTLs under constant-current stressing at $50 \mathrm{~mA} / \mathrm{cm}^{2}$.

To evaluate the effects of $\mathrm{WO}_{3}$ doping on the PhOLED reliability, the above four devices were stressed at a constant current density of $50 \mathrm{~mA} / \mathrm{cm}^{2}$. Their luminance is plotted as a function of the stressing time in Figure 4.13(a), and the normalized data is shown in Figure 4.13(b). The undoped PhOLED exhibits a rapid luminescent decay, which is accompanied by a fast increase in the operation voltage. After just $4 \mathrm{~h}$ stressing, the luminance decreases by $75 \%$, and the voltage increases by $\sim 2.7 \mathrm{~V}$. The half life (defined as the time for the brightness to decline to $50 \%$ of its initial value) under this condition is only $0.27 \mathrm{~h}$. Such a fast decay is caused by significant joule heating at the ITO/undoped CBP interface where there exists a large voltage drop [164], accelerating adverse effects such as impurity diffusion, interfacial reactions, and crystallization of the CBP [165].

The two doped PhOLEDs display much slower luminescent decay and voltage increase. The half life of the PhOLED with $40 \mathrm{~nm}$ doped CBP is improved to $15.6 \mathrm{~h}$, as compared to 13.5 h for the PhOLED with a $10 \mathrm{~nm}$ undoped CBP interlayer between the EML and HTL. The result suggests that diffusion of $\mathrm{WO}_{3}$ into the EML, if occurs, does not accelerate device degradation. Given the large size of $\mathrm{WO}_{3}$ nano-clusters, the dopant diffusion rate could be negligibly slow. 
The stressing behavior of the undoped PhOLED with a $\mathrm{WO}_{3} \mathrm{HIL}$ is also plotted in Figure 4.13(b). Its luminescent decay is slower than that of the undoped device, but faster than those of the doped PhOLEDs. Particularly, compared with the doped PhOLEDs, the device exhibits much faster degradation in the initial stressing stage (the first $2 \mathrm{~h}$ ). It is plausible that this behavior is related to the poor stability of the nanometer-thick HIL at the ITO/CBP interface [166], where a large electric field drives the diffusion of metallic ions and environmental contaminants.

\subsubsection{P-type doping of $\mathrm{CBP}$ with $\mathrm{MoO}_{3}$}

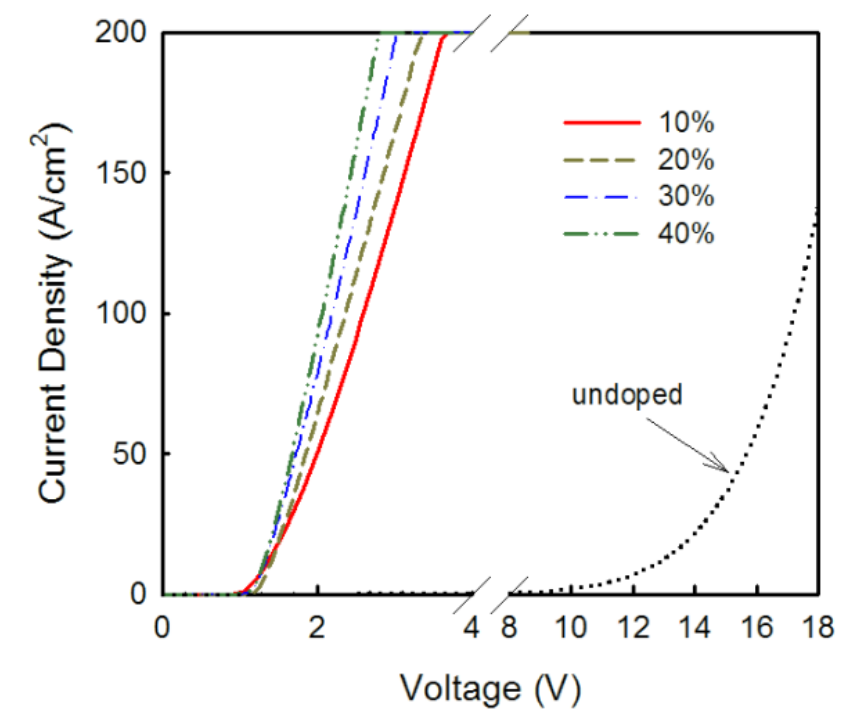

Figure 4.14 J-V characteristics of hole-only devices with CBP doped with $10-40 \mathrm{~mol} \% \mathrm{MoO}_{3}$ as HTL compared to undoped CBP

$\mathrm{MoO}_{3}$ has been chosen as the dopant out of the various oxides due to the ease of processing (thermal evaporation $\mathrm{T}$ ) compared to other oxides, as well as a wide array of fundamental supporting literature available for the $\mathrm{MoO}_{3}$ doped $\mathrm{CBP}$ system [167]. $\mathrm{MoO}_{3}$ was also found to be an excellent p-type dopant for longstanding academic standard HTL of N,N'diphenyl-N,N'-bis-(1-naphthyl)-1-1'-biphenyl-4,4'-diamine ( $\alpha$-NPD), which showed improved efficiency and lifetime for OLEDs with $\mathrm{MoO}_{3}$ doped $\alpha-\mathrm{NPD}$ as transport layer [168-169]. In 
additional to the above advantages, $\mathrm{MoO}_{3}$ doped $\mathrm{CBP}$ is shown to have improved conductivity, [170] forms a CT complex [171], and more critically, excellent injection from ITO without the need for additional surface modification or interlayers. This demonstrates the significant value of integrating $\mathrm{MoO}_{3}$ as a p-type dopant into the novel $\mathrm{CBP}$ architecture. Figure 4.14 shows the current density-voltage (J-V) characteristics of hole-only devices with a CBP HTL uniformly doped with $10-40 \mathrm{~mol}^{2} \mathrm{MoO}_{3}$. As the doping concentration is increased from $10 \mathrm{~mol} \%$ to $40 \mathrm{~mol} \%$, the turn-on voltage barely changes and has a value $\sim 1 \mathrm{~V}$, whereas the differential series resistance decreases from $115 \Omega$ to $76 \Omega$. In contrast, an undoped device has a turn-on voltage greater than $10 \mathrm{~V}$. These results confirm very effective p-type doping of $\mathrm{CBP}$ with $\mathrm{MoO}_{3}$, giving rise to improved hole injection from the ITO anode as well as hole transport inside CBP. The data also suggests that the effect of $\mathrm{MoO}_{3}$ doping in $\mathrm{CBP}$ tends to saturate above $10 \mathrm{~mol} \%$. A similar trend was reported previously for $\mathrm{MoO}_{3}$ doping in TCTA, NPB, and CBP [172-173]. As the dopant concentration was varied from $0-10 \mathrm{~mol} \%$, a sharp increase in the conductivity accompanied by a rapid Fermi level shift toward the host HOMO edge was observed [174].

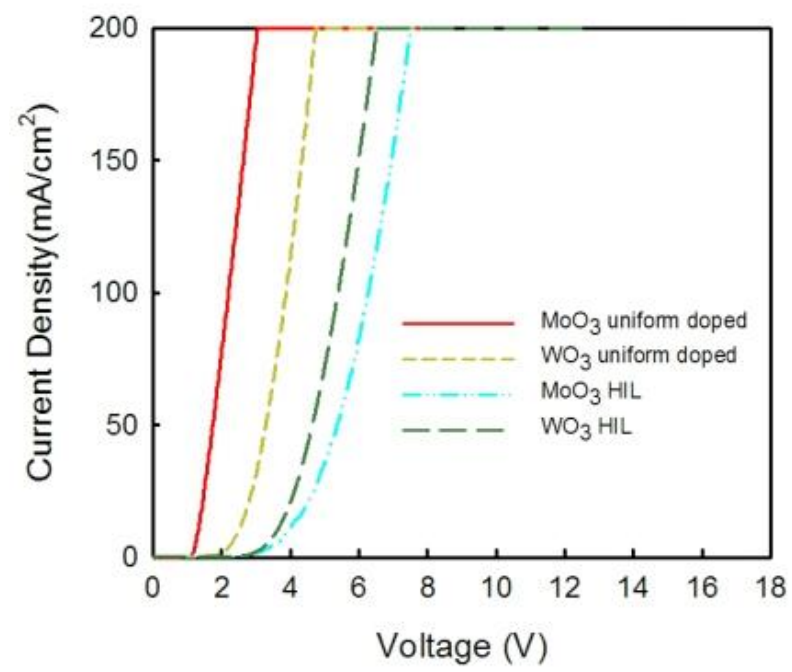

Figure 4.15 J-V characteristics of hole-only devices with a HTL comprising $100 \mathrm{~nm} 30 \mathrm{~mol}^{\circ} \mathrm{MoO}_{3}$ doped CBP, a HTL comprising $100 \mathrm{~nm} 30 \mathrm{~mol} \% \mathrm{WO}_{3}$ doped CBP, $0.5 \mathrm{~nm} \mathrm{MoO} / 100 \mathrm{~nm}$ undoped CBP, or $0.5 \mathrm{~nm}$ $\mathrm{WO}_{3} / 100 \mathrm{~nm}$ undoped CBP.

Figure 4.15 compares injection efficiency and doping efficiency between $\mathrm{MoO}_{3}$ doped 
$\mathrm{CBP}$ and $\mathrm{WO}_{3}$ doped CBP. Similar to $\mathrm{WO}_{3}$, the voltage of $\mathrm{MoO}_{3}$ doped device $(2.21 \mathrm{~V}$ at 100 $\mathrm{mA} / \mathrm{cm}^{2}$ ) is also significantly smaller than that of an undoped device with a $0.5 \mathrm{~nm} \mathrm{MoO}$ hole injection layer (HIL) $\left(6.28 \mathrm{~V}\right.$ at $\left.100 \mathrm{~mA} / \mathrm{cm}^{2}\right)$. At $100 \mathrm{~mA} / \mathrm{cm}^{2}$, the operation voltage of $\mathrm{MoO}_{3}$ doped device $(2.21 \mathrm{~V})$ is smaller than that of $\mathrm{WO}_{3}$ doped device $(3.88 \mathrm{~V})$ and the operation voltage of a $0.5 \mathrm{~nm} \mathrm{MoO}_{3} \mathrm{HIL}(6.28 \mathrm{~V})$ is larger than that of a $0.5 \mathrm{~nm} \mathrm{WO} 3 \mathrm{HIL}(5.38 \mathrm{~V})$. These results suggest that $\mathrm{MoO}_{3}$ doping has a lower efficiency of hole injection yet a higher efficiency of p-type doping than $\mathrm{WO}_{3}$ in CBP. It also confirms that an additional HIL is not needed in OLEDs with a properly doped HTL.

\subsubsection{Doping of $\mathrm{CBP}$ with $\mathrm{ZnO}$ and $\mathrm{SnO}_{2}$}

To verify that p-type doping of wide bandgap CBP with TMOs is due to charge transfer between the HOMO of the organic host and the valence band of the oxide dopant, we designed an experiment to investigate the doping effects of non-transition-metal oxides like $\mathrm{ZnO}$ and $\mathrm{SnO}_{2}$, the electron affinities of $\mathrm{ZnO}(4.3 \mathrm{eV})$ and $\mathrm{SnO}_{2}(4.5 \mathrm{eV})$ are above the HOMO of CBP. Therefore, similar charge transfer would be in the reverse direction in CBP doped with these metal oxides [175].

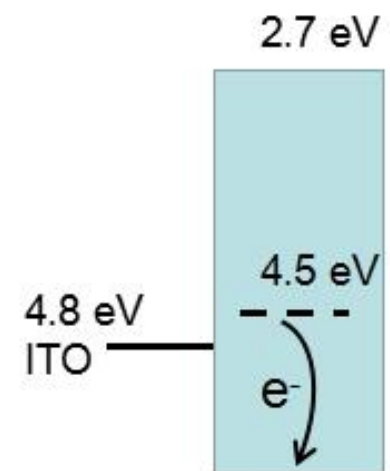

$6.1 \mathrm{eV}$

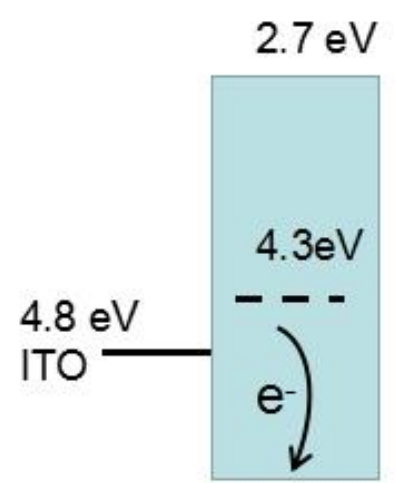

$6.1 \mathrm{eV}$

$\mathrm{SnO}_{2}$ doped CBP

Figure 4.16 Schematic band diagram of the HOMO of CBP and the $\mathrm{CB}$ of $\mathrm{ZnO}$ and $\mathrm{SnO}$. Electron transfer from the $\mathrm{CB}$ of $\mathrm{ZnO}$ and $\mathrm{SnO}_{2}$ to the $\mathrm{HOMO}$ of $\mathrm{CBP}$ results in a reduced hole concentration. 
The schematic band diagram is shown in Figure 4.16. As seen, electrons would transfer from the $\mathrm{VB}$ of $\mathrm{ZnO} \& \mathrm{SnO}_{2}$ to the $\mathrm{HOMO}$ of $\mathrm{CBP}$, reducing the p-type conductivity of the host. Similar hole-only devices consisting of a $0.5 \mathrm{~nm} \mathrm{WO} 3 \mathrm{HIL}, 100 \mathrm{~nm} 30 \mathrm{~mol} \% \mathrm{ZnO}$ and $\mathrm{SnO}_{2}$ doped or undoped CBP HTL, $20 \mathrm{~nm}$ NPB were made to investigate the doping effect.

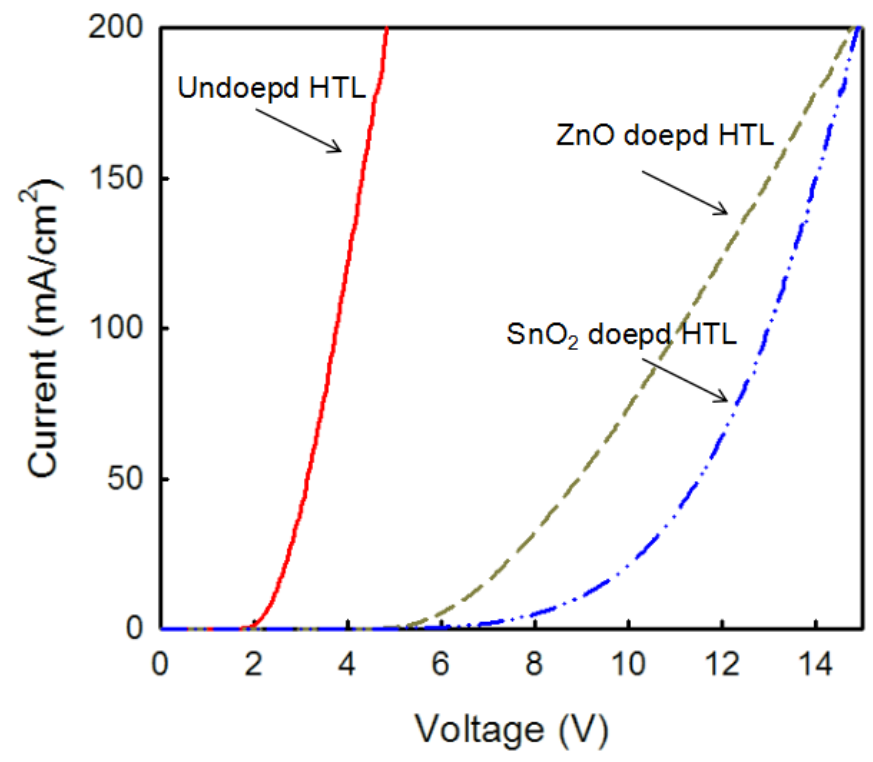

Figure 4.17 J-V curve of hole-only devices with $0.5 \mathrm{~nm} \mathrm{WO} / 100 \mathrm{~nm}$ undoped CBP as HTL compared to 100 nm CBP doped with $30 \% \mathrm{~mol} \mathrm{ZnO}$ and $\mathrm{SnO}_{2}$

Figure 4.17 shows three J-V curves of hole-only devices with an undoped or doped HTL. The undoped device has a low voltage of $2.64 \mathrm{~V}$ at current density of $20 \mathrm{~mA} / \mathrm{cm}^{2}$. However, after doping $\mathrm{ZnO}$ and $\mathrm{SnO}_{2}$ into $\mathrm{CBP}$, the operation voltage rises to $7.28 \mathrm{~V}$ and $9.91 \mathrm{~V}$ at current density of $20 \mathrm{~mA} / \mathrm{cm}^{2}$, respectively. These results are consistent with the above analysis, and the increased operation voltage is mainly caused by electron transfer into the HOMO of the organic host, leading to reduction in the hole concentration.

\subsection{Conclusions}

In summary, we studied the doping of $\mathrm{CBP}$ with $\mathrm{WO}_{3}, \mathrm{MoO}_{3}, \mathrm{SnO}_{2}$ and $\mathrm{ZnO}$, through comparative characterization of hole-only devices. By varying the doping level from 10-40mol\% 
and the doping thickness from 5-40 nm, we have found that, to achieve effective doping with $\mathrm{WO}_{3}$ for improved hole injection and transport, the doped layer should be thicker than $10 \mathrm{~nm}$, and the doping level should be greater than $20 \mathrm{~mol} \%$. It was also found that an energy barrier exists at the doped/undoped CBP interface, resulting in an additional $\sim 1.4 \mathrm{~V}$ voltage drop at 100 $\mathrm{mA} / \mathrm{cm}^{2}$. This finding was explained by a large downward shift of the Fermi level in $\mathrm{WO}_{3}$-doped CBP, which causes band bending and depletion at the interface. Simplified green PhOLEDs with CBP as the HTL and EML host were fabricated. With an appropriately doped HTL, brightness of $11163 \mathrm{~cd} / \mathrm{m}^{2}$ was achieved at $20 \mathrm{~mA} / \mathrm{cm}^{2}$. The corresponding EQE and current efficiency were $13.6 \%$ and $55.8 \mathrm{~cd} / \mathrm{A}$, respectively. The simplified PhOLEDs also exhibited a markedly improved lifetime under constant-current stressing compared to conventional undoped PhOLEDs, and thus represent a viable design for efficient and durable OLEDs suitable for display and lighting applications. Similar results were obtained in $\mathrm{MoO}_{3}$ doped $\mathrm{CBP}$ whereas the critical doping level was $\sim 10 \mathrm{~mol} \%$ and $\mathrm{MoO}_{3}$ showed an even higher doping efficiency than $\mathrm{WO}_{3}$ doping. Finally, a counterexample of $\mathrm{ZnO} \& \mathrm{SnO}_{2}$ doping was done to verify that p-type doping with TMO arises from electrons transfer from the organic host to the metal oxide dopant. 


\section{Chapter 5 Selective Doping of Organic Hole Transport}

\section{Materials with Inorganic Transition Metal Oxides}

\subsection{Introduction}

In Chapter 4, we have demonstrated effective p-type doping of ambipolar wide bandgap hole transport material, $\mathrm{CBP}$, with inorganic $\mathrm{WO}_{3}$ and $\mathrm{MoO}_{3}$ by evaporation co-deposition of the host and dopant materials. The doping effect has been attributed to the formation of charge transfer complexes through electron transfer from the HOMO level of CBP to the conduction band of $\mathrm{WO}_{3}$ or $\mathrm{MoO}_{3}$ nanoclusters. The evaporation co-deposition process generally results in a uniform distribution of the oxide dopants in the organic host [176-178]. However, one drawback of the uniform doping process is that the deposition rate of the dopant must be kept very low but constant, making it very difficult to control [179]. Due to a large contrast in the density between the oxide dopant and organic host, their deposition rates are vastly different. The mathematical equation below expresses how to convert molar doping ratios to deposition rate ratios.

$$
\frac{D_{T M O} \times A \times V_{T M O}}{D_{\mathrm{CBP}} \times A \times V_{C B P}}=\frac{N_{T M O}}{N_{C B P}}=\frac{R}{100 \%-R}
$$

where $\mathrm{D}$ is the deposition rate of each material, A represents the active area which is set to be $1 \mathrm{~cm}^{2}$ for all deposited materials, $\mathrm{V}$ is the molar volume, $\mathrm{N}$ is the number of moles, and $\mathrm{R}$ is the molar ratio.

Table 5.1 lists the molar volumes of two wide bandgap hole transport materials, TCTA and $\mathrm{CBP}$ as well as two p-type dopants, $\mathrm{WO}_{3}$ and $\mathrm{MoO}_{3}$. The molar volume can be obtained from molar mass divided by the density of each material.

Based on equation 5.1, the deposition rate ratios were calculated and listed in Table 5.2 for molar ratios from $10 \%$ to $40 \%$. As see in Table 5.2, the corresponding deposition rate ratio 
would be 0.9:100 for doping CBP with $10 \mathrm{~mol} \% \mathrm{WO}_{3}$. If we deposit $\mathrm{CBP}$ at a normal rate of 0.1 $\mathrm{nm} / \mathrm{s}$ to ensure good uniformity, $\mathrm{WO}_{3}$ needs to be deposited at a small rate of $9 \times 10^{-4} \mathrm{~nm} / \mathrm{s}$. It is very challenging to maintain such a small deposition rate during a typical thermal evaporation process.

Table 5.1 Density, molar mass and molar volume of CBP, TCTA, WO3 and $\mathrm{MoO}_{3}$

\begin{tabular}{|c|c|c|c|}
\hline & Molar mass & Density & Molar volume \\
\hline CBP & $484.59 \mathrm{~g} / \mathrm{mol}$ & $1.199 \mathrm{~g} / \mathrm{cm}^{3}$ & $404.16 \mathrm{~cm}^{3} / \mathrm{mol}$ \\
\hline TCTA & $740.89 \mathrm{~g} / \mathrm{mol}$ & $1.234 \mathrm{~g} / \mathrm{cm}^{3}$ & $600.39 \mathrm{~cm}^{3} / \mathrm{mol}$ \\
\hline $\mathrm{MoO}_{3}$ & $143.94 \mathrm{~g} / \mathrm{mol}$ & $4.69 \mathrm{~g} / \mathrm{cm}^{3}$ & $30.69 \mathrm{~cm}^{3} / \mathrm{mol}$ \\
\hline $\mathrm{WO}_{3}$ & $231.84 \mathrm{~g} / \mathrm{mol}$ & $7.16 \mathrm{~g} / \mathrm{cm}^{3}$ & $32.37 \mathrm{~cm}^{3} / \mathrm{mol}$ \\
\hline
\end{tabular}

Table 5.2 Deposition rate ratio of $\mathrm{WO}_{3}$ vs. $\mathrm{CBP}$ and $\mathrm{MoO}_{3}$ vs. $\mathrm{CBP}$ under molar ratio from $10 \mathrm{~mol} \%$ to $40 \mathrm{~mol} \%$

\begin{tabular}{|c|c|c|}
\hline Molar ratio & Rate ratio $\left(\mathrm{WO}_{3}: \mathrm{CBP}\right)$ & Rate ratio $\left(\mathrm{MoO}_{3}: \mathrm{CBP}\right)$ \\
\hline $10 \%$ & $0.009: 1$ & $0.008: 1$ \\
\hline $20 \%$ & $0.020: 1$ & $0.019: 1$ \\
\hline $30 \%$ & $0.034: 1$ & $0.032: 1$ \\
\hline $40 \%$ & $0.053: 1$ & $0.050: 1$ \\
\hline
\end{tabular}

One strategy to overcome this difficulty is to conduct sequential deposition of the host and dopant materials instead of co-deposition. Given the thermal and kinetic energies carried by the dopant species, they may diffuse into the organic layers, resulting in selective doping [180181]. If the diffusion length is large, a nearly uniform doping profile may be obtained. On the other hand, if the diffusion length is short, dopants are localized in narrow regions of one or a few monolayers, the process is called selective doping, which has been widely used in inorganic semiconductors to produce high sheet conductivity by forming quasi two-dimensional electron 
gas [180-184].

In this chapter, we intend to develop a new doping approach for organic materials through easy sequential deposition. The effects of selective doping are investigated in three hostdopant pairs, $\mathrm{CBP}-\mathrm{WO}_{3}, \mathrm{CBP}-\mathrm{MoO}_{3}$ and TCTA-MoO 3 . The diffusion behaviors of the dopants in the organic hosts are studied by secondary ion mass spectrometry (SIMS). The doping effectiveness is studied and optimized through comparative characterization of hole-only devices and simple $\mathrm{Alq}_{3}$ green OLEDs with a sequentially deposited hole transport layer.

\subsection{Experimental Procedure}

(a)

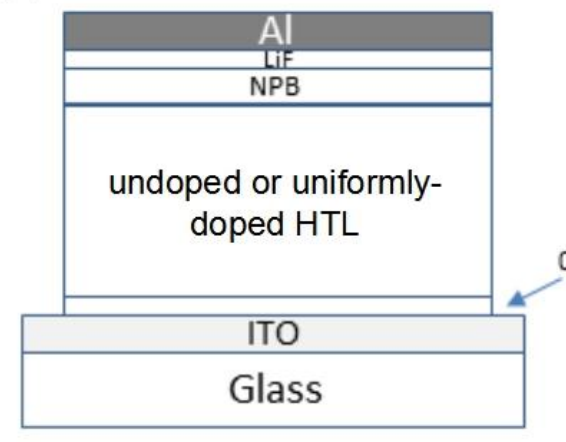

(b)

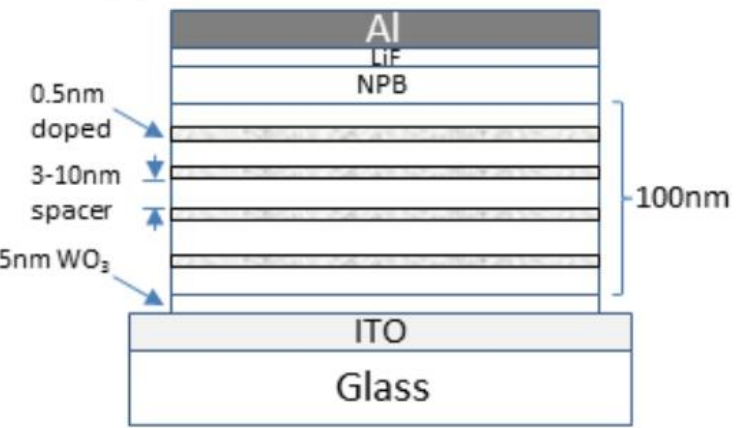

Figure 5.1 Schematic layer structures of hole-only devices with (a)uniformly doped HTL and (b) selectively doped HTL. Both hole-only devices have same $0.5 \mathrm{~nm} \mathrm{WO}$ as HIL.

Hole-only devices with a selectively-doped HTL consisting of alternative undoped organic spacer $(3-10 \mathrm{~nm})$ and $0.5 \mathrm{~nm}$ TMO layers were made. For comparison, hole-only devices with $100 \mathrm{~nm}$ uniformly doped HTL were also prepared. A schematic cross-sectional view of the selectively-doped and uniformly doped devices is given in Figure 5.1.

Figure 5.2 shows the layer structure of the simple green OLEDs consisting of a $0.5 \mathrm{~nm}$ HIL, a $60 \mathrm{~nm}$ undoped or selectively-doped HTL, a $40 \mathrm{~nm}$ Alq 3 layer, and a $0.5 \mathrm{~nm} \mathrm{LiF/100} \mathrm{nm}$ Al cathode. The surface morphology of doped and undoped organic films was characterized by AFM in the tapping mode. The film transparency was compared via transmission measurements. 
Hole-only and green OLED devices were fabricated on glass substrates with pre-patterned ITO (sheet resistance $\sim 20 \Omega / \square$ ).

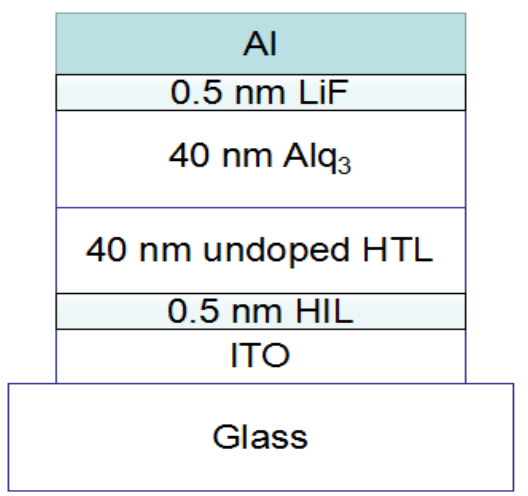

(a)

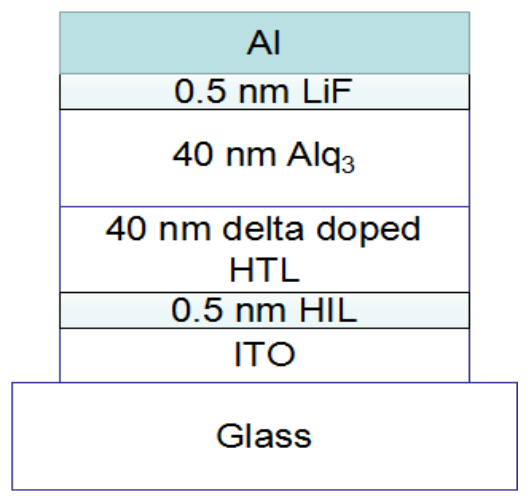

(b)

Figure 5.2 Schematic layer structures of green Alqu OLEDs with (a) uniformly doped HTL and (b) selectively doped HTL. Both OLED devices have a same $0.5 \mathrm{~nm} \mathrm{WO}_{3} \mathrm{HIL}$.

The substrates were first cleaned with solvents and deionized water, and exposed to $\mathrm{O} 2$ plasma for $5 \mathrm{~min}$. They were then transferred to a thermal evaporation system with a base pressure of $\sim 1 \times 10^{-7}$ Torr for deposition of organic materials, $\mathrm{MoO}_{3}, \mathrm{LiF}$ and $\mathrm{Al}$ at pre-calibrated rates. The electroluminescence spectrum and luminance of the OLEDs were measured as a function of injection current density in the range of $10^{-4}-10^{-1} \mathrm{~A} / \mathrm{cm}^{2}$. Both the simple OLEDs and hole-only devices had an active area of $0.1 \mathrm{~cm}^{2}$. The fabricated devices were encapsulated with glass lids in a $\mathrm{N}_{2}$-filled glovebox and characterized electrically and optically at room temperature. To evaluate the device reliability, the OLEDs were placed directly onto the surface of a calibrated photodetector and stressed at a constant current density of $100 \mathrm{~mA} / \mathrm{cm}^{2}$ while their luminance and voltage were recorded every 5 seconds.

\subsection{Results and Discussion}

\subsubsection{Selective Doping of CBP with $\mathrm{WO}_{3}$}

AFM scans of $100 \mathrm{~nm}$ doped and undoped CBP films over a $5 \mu \mathrm{m} \times 5 \mu \mathrm{m}$ area revealed 
similar film surfaces which appear to be amorphous and have no distinctive morphological features as shown in Figure 5.3. The root-mean-square surface roughness of the undoped film is $1.51 \mathrm{~nm}$, whereas the roughness values of the uniformly-doped and selectively-doped (with $3 \mathrm{~nm}$ undoped spacer layers) samples are $1.46 \mathrm{~nm}$ and $1.75 \mathrm{~nm}$, respectively. Clearly, $\mathrm{WO}_{3}$ doping does not cause a significant change in the film morphology, and in the case of uniform doping, it even smooths the surface a little bit. It is plausible that the heavy $\mathrm{WO}_{3}$ molecules or nanoclusters can help to block void defects and densify the porous organic films.
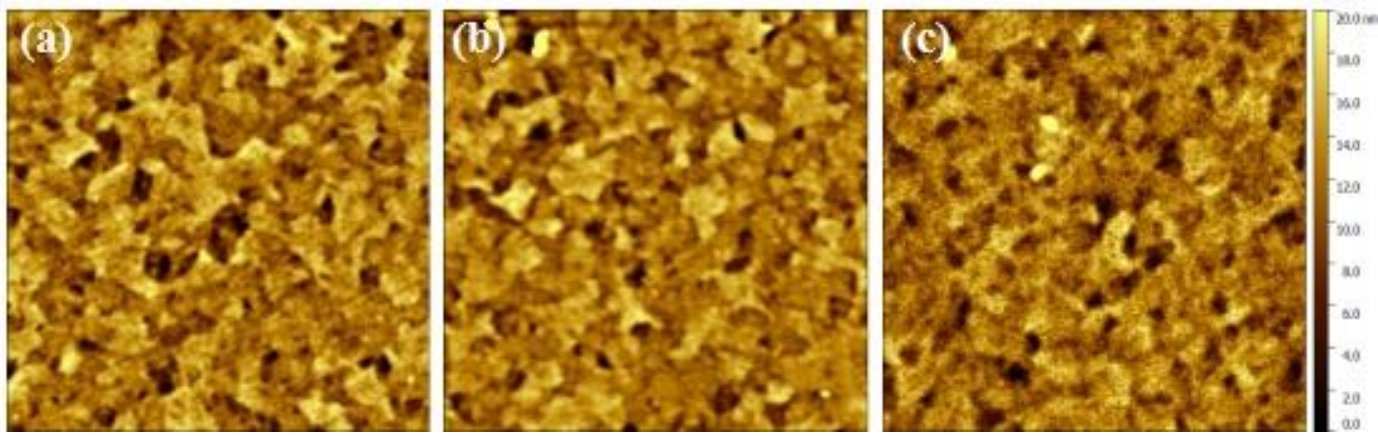

Figure 5.3 AFM scans of $100 \mathrm{~nm}$ CBP films over a $5 \mu \mathrm{m} \times 5 \mu \mathrm{m}$ area. Film (a) was undoped, film (b) was doped with 30 $301 \% \mathrm{WO}_{3}$ by co-deposition and film (c) was doped by sequential deposition of alternative 0.5 nm $\mathrm{WO}_{3}$ and $3 \mathrm{~nm}$ CBP layers.

Figure 5.4(a) shows the current density-voltage $(\mathrm{J}-\mathrm{V})$ characteristics of hole-only devices with a periodically selectively-doped CBP HTL. The $\mathrm{WO}_{3}$ delta-doping level was varied from 40-100mol\%, whereas the undoped spacer layer thickness was fixed at $3 \mathrm{~nm}$. As compared to an undoped device, the doped devices have a smaller forward voltage indicating enhanced hole transport resulting from selective doping. With $40 \mathrm{~mol} \%$ and $60 \mathrm{~mol} \%$ doping, the voltage is slightly reduced. The respective voltages at $100 \mathrm{~mA} / \mathrm{cm}^{2}$ are $5.3 \mathrm{~V}$ and $5.0 \mathrm{~V}$, as compared to 5.8 $\mathrm{V}$ for the undoped device. As the doping level is increased to $80 \mathrm{~mol} \%$ and $90 \mathrm{~mol} \%$, the voltage is substantially reduced, down to $3.4 \mathrm{~V}$ and $2.8 \mathrm{~V}$, respectively. The value is further reduced to $\sim 2.1 \mathrm{~V}$ for the $100 \mathrm{~mol} \%$ selectively-doped device, where the HTL comprises periodic $0.5 \mathrm{~nm}$ thick neat $\mathrm{WO}_{3}$ layers. Figure 5.4(b) displays the device characteristics on the log-log scale. Three distinct regions can be identified in all the $\mathrm{J}-\mathrm{V}$ curves: an ohmic region $(\mathrm{J} \propto \mathrm{V})$ at low 
voltages, a trap-charge limited-current region $\left(\mathrm{J} \propto \mathrm{V}^{\mathrm{n}}, \mathrm{n}>2\right)$ at intermediate voltages, and a spacecharge limited-current region $\left(\mathrm{J} \propto \mathrm{V}^{2}\right)$ at high voltages [185]. The result suggests that the current flow in the undoped and doped devices may be ascribed to the same hole transport mechanism.

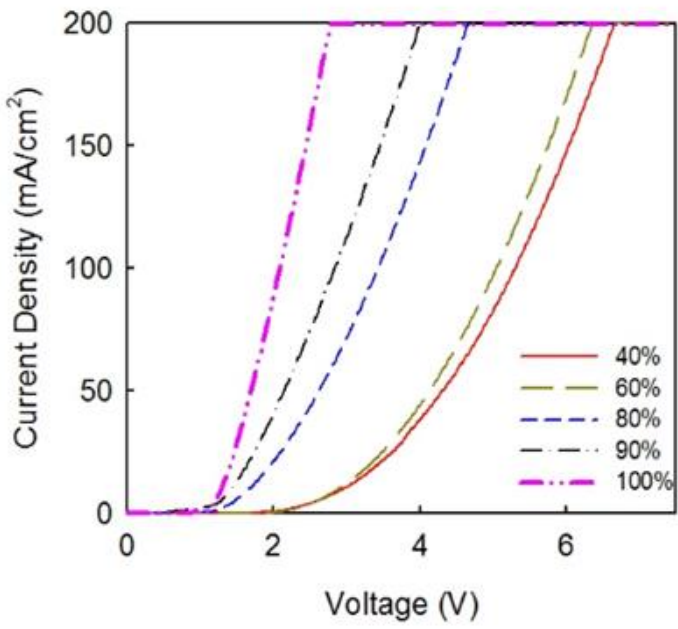

(a)

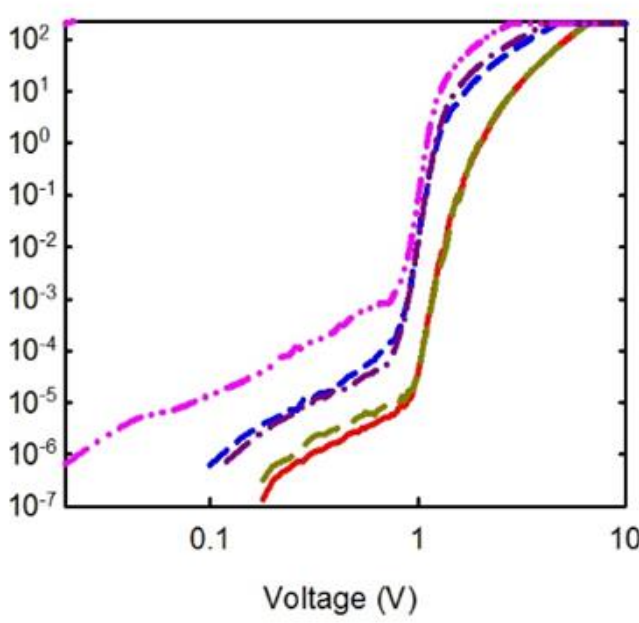

(b)

Figure 5.4 (a)J-V characteristics of hole-only devices with a $100 \mathrm{~nm}$ CBP HTL comprising alternative $3 \mathrm{~nm}$ undoped spacer and $0.5 \mathrm{~nm}$ 40-100mol\% WO 3 -doped CBP layers. (b)J-V data plotted on the log-log scale.

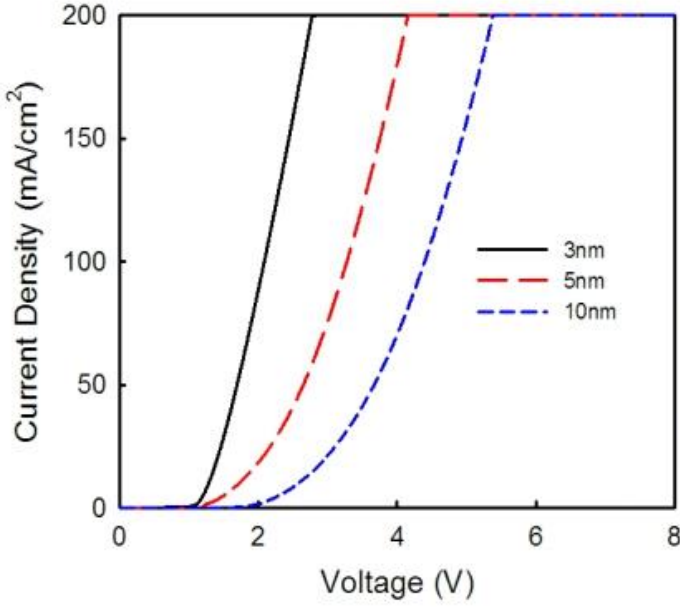

(a)

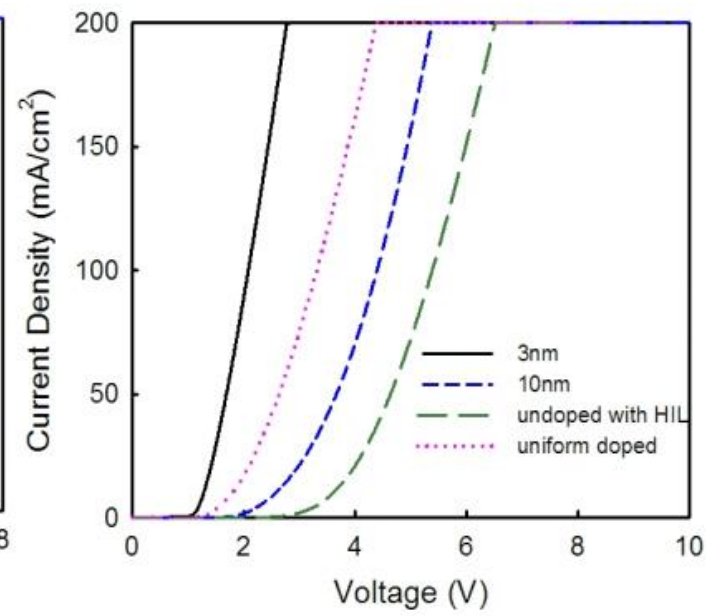

(b)

Figure 5.5 (a)J-V characteristics of hole-only devices with a $100 \mathrm{~nm}$ doped CBP HTL comprising alternative 3-10 $\mathrm{nm}$ undoped spacer and $0.5 \mathrm{~nm} \mathrm{WO}$ layers prepared by sequential deposition. (b)J-V characteristics of hole-only devices with a $100 \mathrm{~nm} 67 \mathrm{~mol} \%$ WO $_{3}$ doped CBP HTL and a $0.5 \mathrm{~nm} \mathrm{WO} / 100 \mathrm{~nm}$ undoped CBP as HTL

To investigate the influence of the undoped spacer on the doping effect, selectively doped 
hole-only devices comprising alternative $0.5 \mathrm{~nm} \mathrm{WO}_{3}$ and 3-10 $\mathrm{nm}$ undoped CBP spacer were fabricated. The J-V characteristics of the devices are compared in Figure 5.5(a). The device with $3 \mathrm{~nm}$ undoped spacer layers has a remarkably small turn-on voltage $\sim 1 \mathrm{~V}$ and a forward voltage of $2.1 \mathrm{~V}$ at $100 \mathrm{~mA} / \mathrm{cm}^{2}$. As the spacer thickness is increased to $5 \mathrm{~nm}$, the voltage increases to 3.3 $\mathrm{V}$. It further increases to $4.4 \mathrm{~V}$ for the device with $10 \mathrm{~nm}$ spacer. The differential series resistances of these three devices are $70 \Omega, 83 \Omega$, and $94 \Omega$, respectively.

For comparison, hole-only devices with a $0.5 \mathrm{~nm} \mathrm{WO} 3 \mathrm{HIL} / 100 \mathrm{~nm}$ undoped CBP HTL or a $100 \mathrm{~nm}$ CBP HTL uniformly doped with $67 \mathrm{~mol}_{\%} \mathrm{WO}_{3}$ equivalent to the total mole ratio of selectively doped CBP with $3 \mathrm{~nm}$ spacer, were also fabricated. Their J-V curves are shown together with selective doping curves in Figure 5.5(b). As seen, even with same molar ratio of $\mathrm{WO}_{3}$, the selectively-doped devices with $3 \mathrm{~nm}$ spacer exhibit better conductivity and have a lower forward voltage. Meanwhile, the device with $10 \mathrm{~nm}$ spacer has a voltage higher than that of the uniformly doped device, but considerably lower than that of the undoped device. Note that all the currents flow vertically between the anode and cathode, in a direction perpendicular to the film plane of the selectively-doped HTL. Given that selective doping is conventionally applied to inorganic materials to achieve enhanced conductivity along the film plane of the doped structure, the above finding that periodic selective doping leads to higher vertical current conduction in organic materials is quite striking.

The remarkable effectiveness of selective doping may arise from dopant diffusion which results in a nearly uniform layer with a high nominal doping concentration. To confirm this point, SIMS measurements were conducted to investigate diffusion of $\mathrm{WO}_{3}$ into $\mathrm{CBP}$, which determines the actual doping profile in the selectively-doped samples. To facilitate SIMS profiling, we prepared a thin film consisting of alternative $15 \mathrm{~nm} \mathrm{CBP/3} \mathrm{nm} \mathrm{WO}$. The tungsten profile is shown in Figure 5.6. Due to vastly different sputtering rates of these two materials, which cause strong atomic mixing effects and roughening of the analyzed area, a good depth resolution is hard to obtain and as seen, the resolution inevitably degrades as profiling proceeds. 
Therefore, our analysis is focused on the top three $\mathrm{CBP} / \mathrm{WO}_{3} / \mathrm{CBP}$ layers.

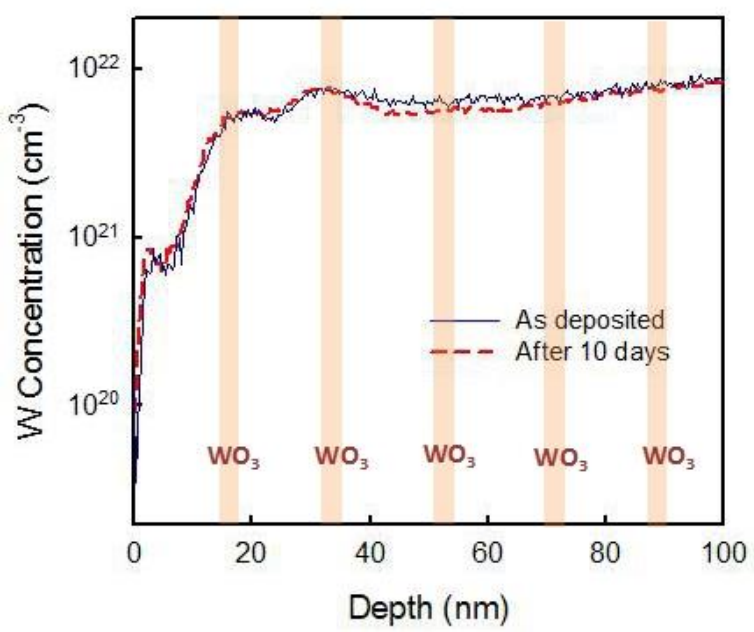

Figure 5.6 SIMS depth profile of tungsten in alternative $3 \mathrm{~nm} \mathrm{WO} / 15 \mathrm{~nm}$ CBP thin films

A nearly uniform doping profile with only two weak peaks is seen and retesting taken after 10 days storage in air produces an identical profile. This is consistent with the finding by Zhao et al. that "hot" metal oxide clusters diffuse deep into the underlying organic material [186]. Since the glass transition temperature of $\mathrm{CBP}$ is only $62^{\circ} \mathrm{C}$ and the thermal evaporation temperature of $\mathrm{WO}_{3}$ in high vacuum $\left(\sim 10^{-7}\right.$ torr $)$ is $\sim 900{ }^{\circ} \mathrm{C}$, diffusion length of $\mathrm{WO}_{3}$ in underlying CBP should be much more than $10 \mathrm{~nm}$. In other words, alternative yet simple method of uniform doping is achieved by selective doping of CBP with $\mathrm{WO}_{3}$.
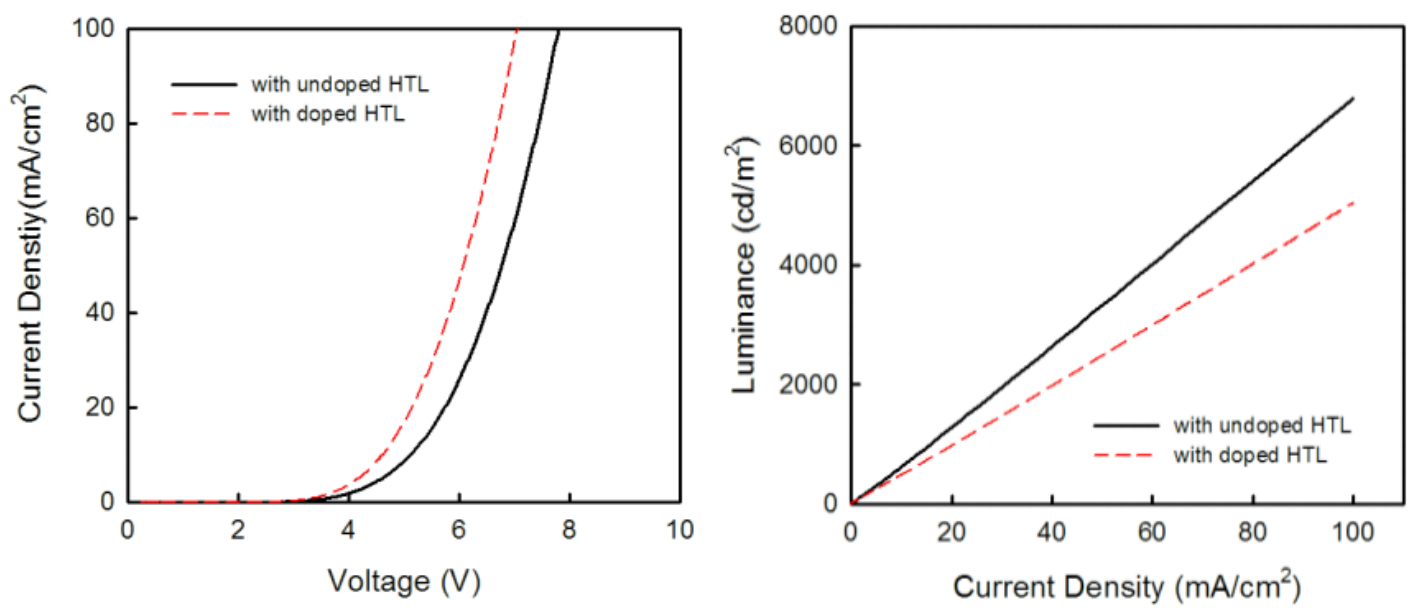

Figure 5.7 (a)J-V and (b)L-I characteristics of green OLEDs with an undoped CBP HTL or a CBP doped with $\mathrm{WO}_{3}$ HTL by sequential deposition. 
Figure 5.7 compares the current density-voltage $(\mathrm{J}-\mathrm{V})$ and luminance-current density (LJ) characteristics of $\mathrm{Alq}_{3}$ OLEDs with a $0.5 \mathrm{~nm} \mathrm{WO} / 60 \mathrm{~nm}$ undoped or $60 \mathrm{~nm}$ selectively doped CBP HTL. From Figure 5.7(a), the device with an undoped HTL has a high operation voltage of $5.7 \mathrm{~V}$ at $20 \mathrm{~mA} / \mathrm{cm}^{2}, 0.6 \mathrm{~V}$ higher than that of the selectively-doped OLED. Since electron injection and transport as well as hole injection are the same in these two OLEDs, the voltage reduction must arise from improved hole transport in selectively-doped HTL. As seen in Figure $5.7(\mathrm{~b})$, at the current density of $20 \mathrm{~mA} / \mathrm{cm}^{2}$, the values of green luminance of the devices are 1268 and $969 \mathrm{~cd} / \mathrm{m}^{2}$, respectively.

The corresponding current efficiencies are 6.34 and $4.85 \mathrm{~cd} / \mathrm{A}$. The OLED with an undoped HTL is slightly brighter presumably due to more balanced hole and electron transport. With an electron mobility $\sim 8 \times 10^{-6} \mathrm{~V}^{-1} \mathrm{~cm}^{2} / \mathrm{s}$, Alq 3 has very poor electron transport capability, which may balance better poor hole transport within the undoped CBP HTL. Another factor limiting the luminance in the selectively-doped OLED is the relatively lower transparency of the layered HTL structure. Figure 5.8 illustrates that the transmission of a $60 \mathrm{~nm}$ selectively-doped CBP at $540 \mathrm{~nm}$ was found to be $90.8 \%$ as compared to $98.4 \%$ for a $60 \mathrm{~nm}$ undoped CBP. The transmission loss is attributed to light reflection at multiple interfaces between undoped and doped CBP.

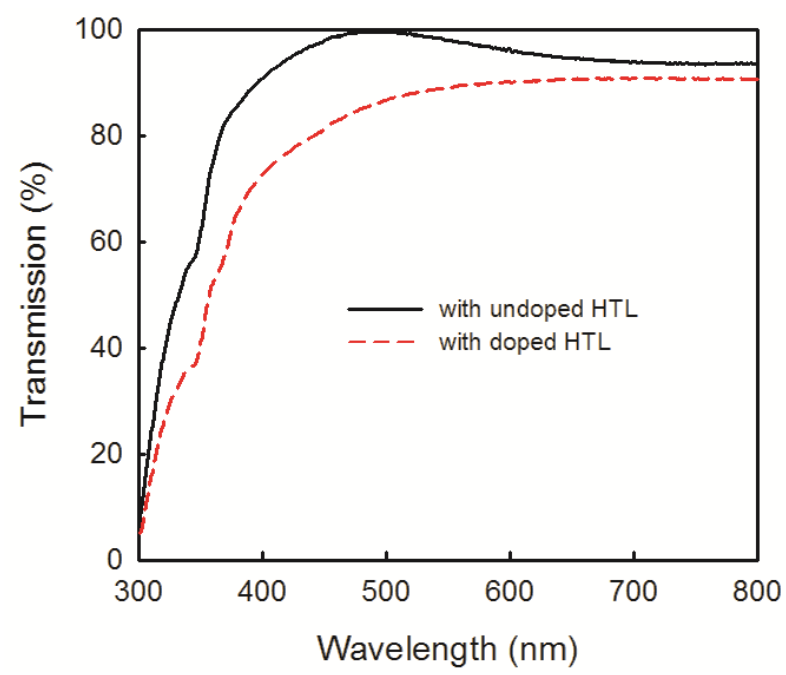

Figure 5.8 Transmission of thin films comprising $0.5 \mathrm{~nm} \mathrm{WO}_{3} / 60 \mathrm{~nm}$ undoped $\mathrm{CBP}$ or $60 \mathrm{~nm}$ sequential doped $\mathrm{CBP}$ with $\mathrm{WO}_{3}$ 


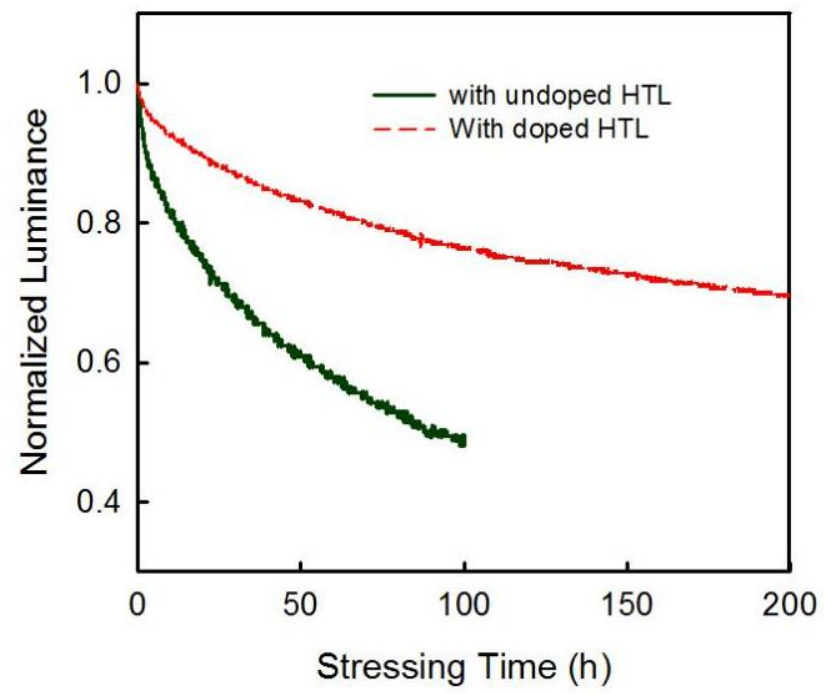

Figure 5.9 Evolution of the normalized luminance of OLEDs with undoped and doped HTL stressed at a constant current density of $100 \mathrm{~mA} / \mathrm{cm}^{2}$

The selectively doped device also exhibited better reliability under stressing at a constant current density of $100 \mathrm{~mA} / \mathrm{cm}^{2}$. Their normalized luminance is plotted as a function of the stressing time in Figure 5.9. The undoped OLED exhibits a rapid luminescent decay, which is accompanied by a steady increase in the voltage. $T_{0.7}$, defined as the time for the brightness to decline to $70 \%$ of its initial value, is $28 \mathrm{~h}$. The voltage increases by $\sim 0.4 \mathrm{~V}$ during this period. The doped OLED displayed much slower luminescent decay and smaller voltage increase. The $\mathrm{t}_{0.7}$ of the OLED is improved to $195 \mathrm{~h}$, and the voltage increases by $1.2 \mathrm{~V}$ over the entire stressing period. Since the two devices only differed in their HTL structure, the slower decay in the latter indicates slower degradation of the selectively-doped CBP HTL than the undoped HTL. This could be attributed to reduced power dissipation and suppressed thermally-induced reactions within the doped organic structure [187].

\subsubsection{Selective Doping of CBP with $\mathrm{MoO}_{3}$}

The J-V characteristics of hole-only devices with a selectively-doped CBP HTL 
comprising alternative $0.5 \mathrm{~nm} \mathrm{MoO}_{3}$ and undoped CBP spacer layers are plotted in Figure 5.10. To investigate the influence of the undoped spacer on current conduction, the spacer thickness was varied from 3 to $10 \mathrm{~nm}$. Also, plotted in Figure 5.10 are the J-V curves of devices with an undoped or uniformly-doped CBP HTL.

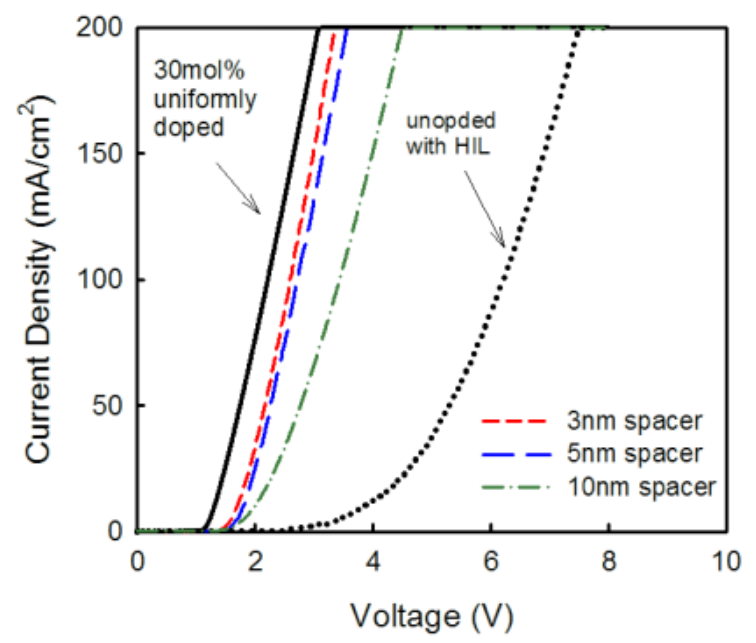

Figure 5.10 Evolution of the normalized luminance of OLEDs with undoped and selectively-doped HTL stressed at a constant current density of $100 \mathrm{~mA} / \mathrm{cm}^{2}$

As seen, compared with the undoped device, all the selectively-doped devices have a markedly reduced forward voltage. Since all the devices have a $0.5 \mathrm{~nm} \mathrm{MoO} 3 \mathrm{HIL}$ and thus the same hole injection behavior, the voltage reduction must result from enhanced charge transport through the selectively doped HTL. The devices with $3 \mathrm{~nm}$ or $5 \mathrm{~nm}$ spacer have almost identical $\mathrm{J}-\mathrm{V}$ characteristics with a low turn-on voltage of $1.5 \mathrm{~V}$ and a small series resistance of $92 \Omega$ suggesting a saturated effect of selective doping for thin spacer layers. Figure 5.10 also shows that the device with a uniformly-doped HTL has a slightly lower turn-on voltage than the best selectively-doped device. This may be attributed to more efficient hole injection at the ITO/doped CBP interface, or the combined effect of improved hole injection and transport.

Figure 5.11 compares the current density-voltage $(\mathrm{J}-\mathrm{V})$ and luminance-current density (LJ) characteristics of $\mathrm{Alq}_{3}$ OLEDs with the same HIL and two different types of HTLs: $60 \mathrm{~nm}$ undoped $\mathrm{CBP}$ and $60 \mathrm{~nm} \mathrm{MoO}_{3}$ selectively doped $\mathrm{CBP}$ with $3 \mathrm{~nm}$ spacer. As seen in Figure 
5.11(a), the device with an undoped HTL has an operation voltage of $5.7 \mathrm{~V}$ at $20 \mathrm{~mA} / \mathrm{cm}^{2}, 0.9 \mathrm{~V}$ higher than that of the OLED with a selectively-doped HTL. Since the behaviors of electron injection and transport as well as hole injection are identical in these two OLEDs, the voltage reduction must arise from improved hole transport in the selectively-doped HTL. At $20 \mathrm{~mA} / \mathrm{cm}^{2}$, the values of green luminance of the devices are 1155 and $855 \mathrm{~cd} / \mathrm{m}^{2}$, respectively. The corresponding current efficiencies are 5.78 and $4.28 \mathrm{~cd} / \mathrm{A}$ as shown in Figure 5.11(b).
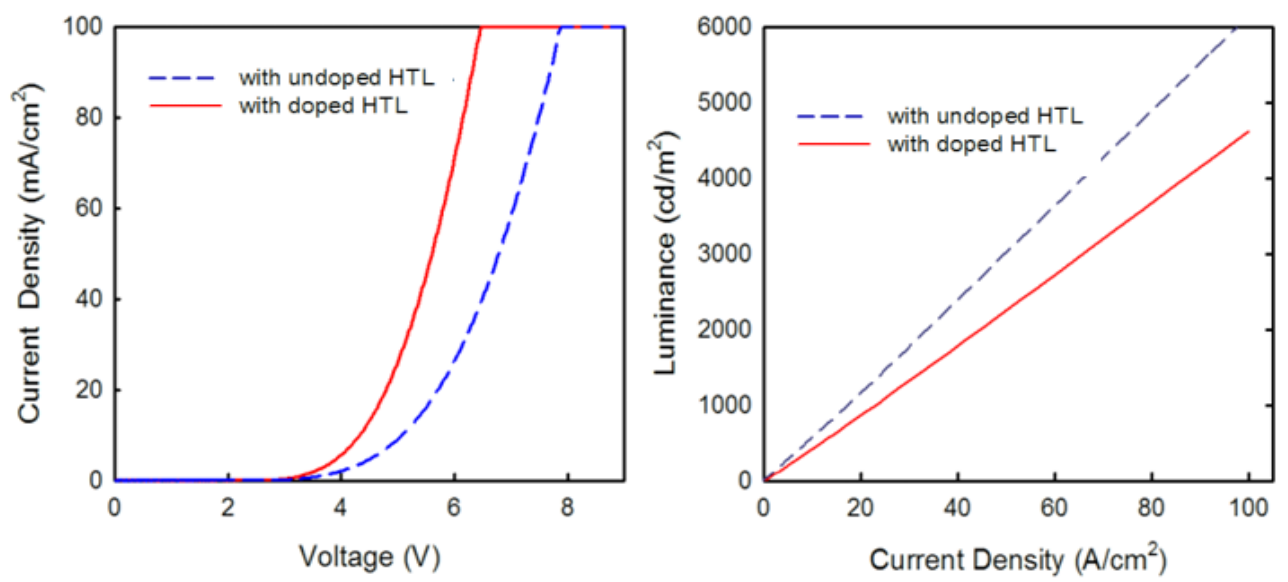

Figure 5.11 (a) J-V and (b)L-J characteristic of OLEDs comprising undoped $\mathrm{CBP}$ as $\mathrm{HTL}$ and $\mathrm{MoO}_{3}$ selectively doped CBP as HTL

Like selective doping of CBP with $\mathrm{WO}_{3}$, the OLED device with undoped HTL is $35 \%$ brighter than with selectively doped HTL, presumably due to more balanced hole and electron transport. Also, the lower transparency of the doped HTL due to light reflection at the undoped/doped interfaces limits the luminance of the $\mathrm{MoO}_{3}$ selectively-doped OLED. The transmission of a $60 \mathrm{~nm}$ selectively doped CBP at the $540 \mathrm{~nm}$ peak wavelength of the OLEDs was also measured to be $88 \%$ as compared to $98 \%$ for a $60 \mathrm{~nm}$ undoped CBP.

Similarly, to evaluate the influence of selectively doping on the OLED reliability, the above two devices were stressed at a constant current density of $100 \mathrm{~mA} / \mathrm{cm}^{2}$. Their normalized luminance is plotted as a function of the stressing time in Figure 5.12. The undoped OLED exhibits a rapid luminescent decay, which is accompanied by a significant increase in the voltage. The $50 \%$ lifetime, $\mathrm{t}_{0.5}$, defined as the time for the brightness to decline to $50 \%$ of its initial value, 
is $18 \mathrm{~h}$. The voltage increases by $\sim 0.5 \mathrm{~V}$ during this period. The doped OLED displays much slower luminescence decay and less voltage increase. The $\mathrm{t}_{0.5}$ of the OLED is improved to $42 \mathrm{~h}$, and the voltage increases by $0.4 \mathrm{~V}$ over the entire stressing period. Since the two devices only differed in their HTL structures like selective doping of $\mathrm{CBP}$ with $\mathrm{WO}_{3}$, the slower decay in the latter indicates slower material degradation of the selectively-doped CBP HTL than the undoped HTL as discussed in Section 5.3.2.

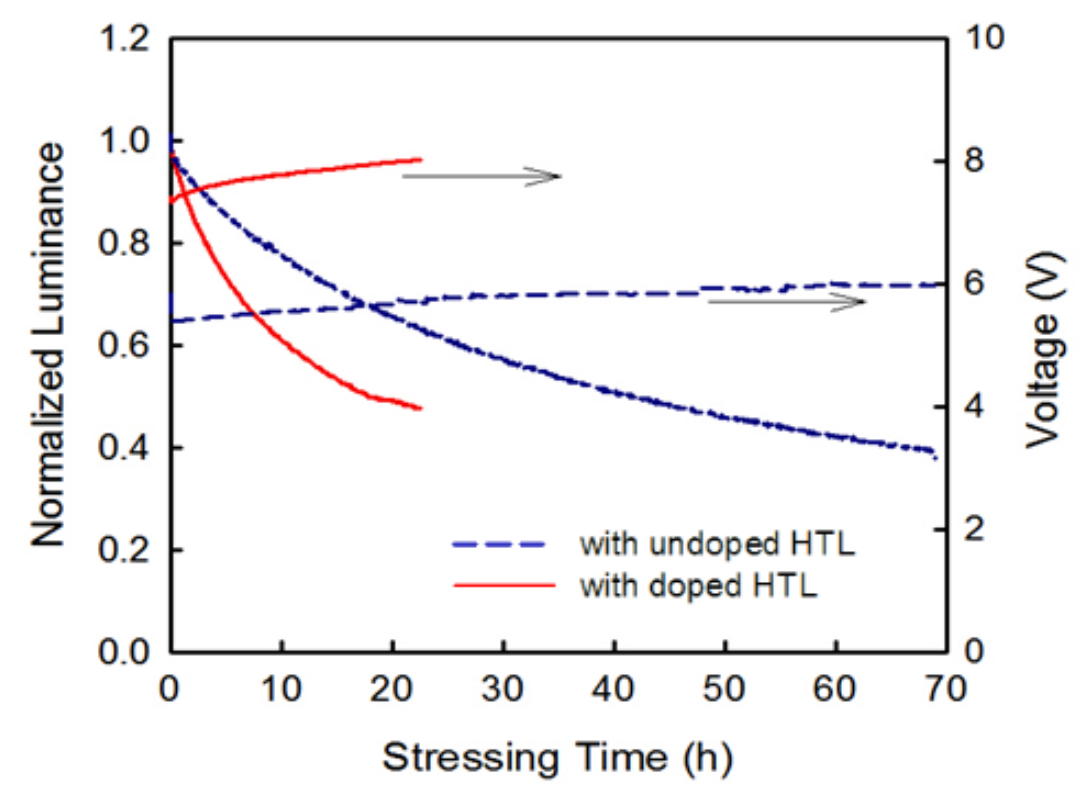

Figure 5.12 Evolution of the normalized luminance and operation voltage of OLEDs with an undoped or doped HTL stressed at a constant current density of $100 \mathrm{~mA} / \mathrm{cm}^{2}$

\subsubsection{Selective Doping of TCTA with $\mathrm{MoO}_{3}$}

As discussed in previous sections, diffusion of metal oxides in organic materials with a low glass transition temperature could be very fast, smoothing out the doping profile and leading to the same effects as uniform doping. However, TCTA has a glass transition temperature of 152 ${ }^{\circ} \mathrm{C}$ which is much higher than that of many organic materials, and thus creates a higher diffusion barrier for the TMO dopant which may cause the formation of delta doping. To validate this, SIMS measurements were also conducted to investigate diffusion of $\mathrm{MoO}_{3}$ into TCTA, which 
determines the actual doping profile in the selectively-doped samples. To facilitate SIMS profiling, we prepared a thin film consisting of alternative $15 \mathrm{~nm}$ TCTA/3 nm MoO 3 . The Mo profile is shown in Figure 5.13. Similar to previous SIMS measurements, our analysis is focused on the top three TCTA/MoO$/$ TCTA layers.

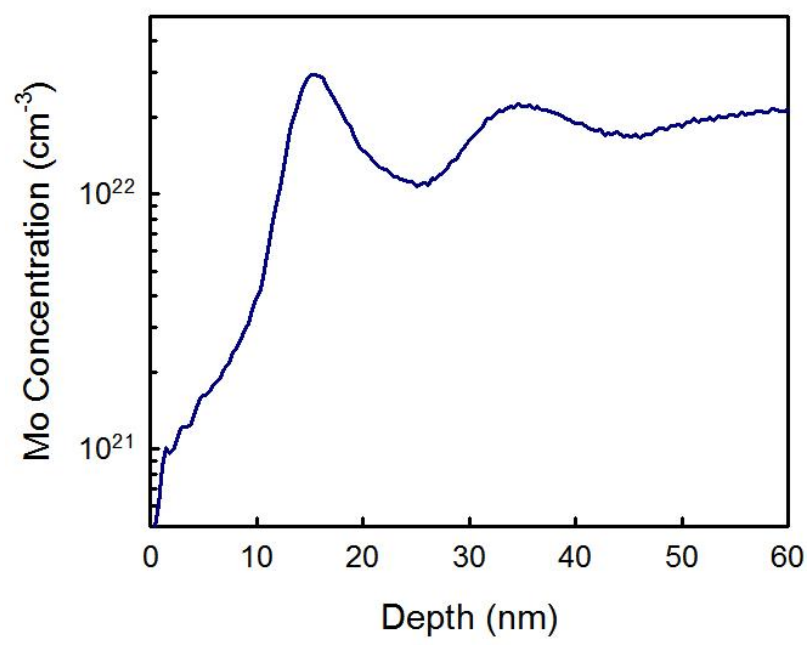

Figure 5.13 SIMS depth profile of Mo in alternative $3 \mathrm{~nm} \mathrm{MoO}_{3} / 15 \mathrm{~nm}$ TCTA thin films

A sharp asymmetrical Mo peak extending into overlying and underlying TCTA layers is seen in Figure 5.13. The respective characteristic diffusion lengths extracted from the profile are $\sim 1.4 \mathrm{~nm}$ and $4 \mathrm{~nm}$, which overestimate the actual diffusion lengths given the above-mentioned atomic mixing and surface roughening effects. This result suggests minimal diffusion of Mo into the overlying TCTA layer. The tail of the Mo peak in the underlying TCTA is likely caused by transient diffusion driven by the thermal and kinetic energies carried by the evaporated $\mathrm{MoO}_{3}$ species. Since the diffusion length of $\mathrm{MoO}_{3}$ in underlying TCTA may be well below $4 \mathrm{~nm}$, periodic spatial fluctuations of the dopant concentration must exist in the selectively-doped samples. In other words, delta doping is achieved through sequential evaporation deposition of neat TCTA and $\mathrm{MoO}_{3}$.

Figure 5.14(a) shows the current density-voltage (J-V) characteristics of hole-only devices with an undoped TCTA HTL or a TCTA HTL uniformly doped with $10-40 \mathrm{~mol} \% \mathrm{MoO}_{3}$. The undoped device has a voltage of $9 \mathrm{~V}$ at $100 \mathrm{~mA} / \mathrm{cm}^{2}$. With $10 \mathrm{~mol} \%$ doping, the voltage is 
reduced to $5.7 \mathrm{~V}$. It is further decreased to $4.3 \mathrm{~V}$ and $3.0 \mathrm{~V}$ for $20 \mathrm{~mol} \%$ and $30 \mathrm{~mol} \%$ doping, respectively. As the doping concentration is raised to from $30 \mathrm{~mol} \%$ to $30-40 \mathrm{~mol} \%$, the voltage is only reduced slightly by $0.3 \mathrm{~V}$, whereas the turn-on voltage is essentially unchanged at $1.1 \mathrm{~V}$. This trend suggests that the effect of $\mathrm{MoO}_{3}$ doping in TCTA is saturated at a level $40 \mathrm{~mol} \%$.
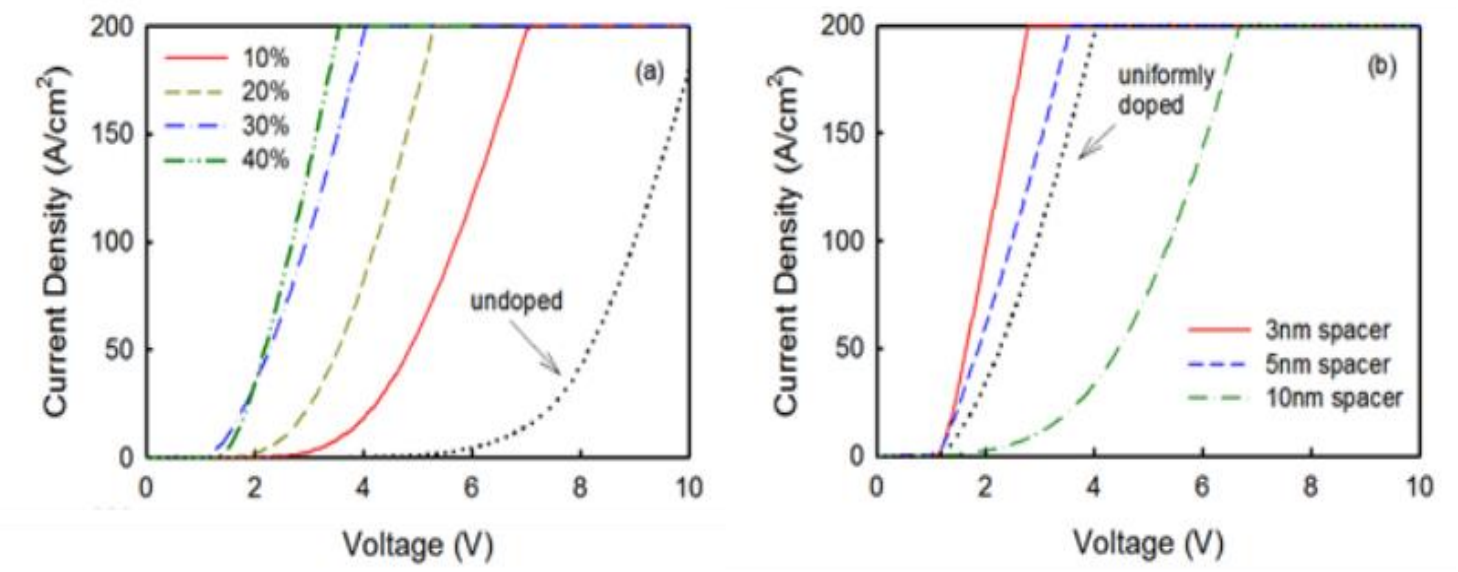

Figure 5.14 J-V characteristic of hole-only devices with a (a)undoped/MoO $\mathrm{M}_{3}$ doped TCTA HTL or (b)MoO delta-doped TCTA HTL

Figure 5.14(b) displays the J-V characteristics of hole-only devices with a delta-doped TCTA HTL comprising alternative $0.5 \mathrm{~nm} \mathrm{MoO}_{3}$ and undoped TCTA spacer layers. To investigate the influence of the undoped spacer on current conduction, the spacer thickness was varied from 3 to $10 \mathrm{~nm}$. As seen, compared with the undoped device, all the delta-doped devices have a markedly reduced forward voltage. Since all the devices have an identical $0.5 \mathrm{~nm} \mathrm{MoO}_{3}$ HIL, the voltage reduction must result from enhanced charge transport through the delta-doped HTL. It is striking that the delta-doped devices with $3 \mathrm{~nm}$ or $5 \mathrm{~nm}$ spacer layers have an even smaller forward voltage than the $30 \mathrm{~mol} \%$ uniformly doped device. The best doping effect is obtained in the device with $3 \mathrm{~nm}$ spacer, which has an extremely low turn-on voltage of $1 \mathrm{~V}$ and a small series resistance of $76 \Omega$. Note that all the measured currents flow vertically through the device layer structure between the anode and cathode, in a direction perpendicular to the film plane of the delta-doped HTL. It can thus be concluded that delta doping greatly improves charge transport in a direction perpendicular to the doped layers, and the conductivity increases as the 
undoped organic spacer layers are shrunk.

In previous sections, selective doping of $\mathrm{CBP}$ with $\mathrm{WO}_{3}$ and $\mathrm{MoO}_{3}$ resulted in a greater improvement in conductivity than uniform doping. This is similar to the case of delta doping of TCTA with $\mathrm{MoO}_{3}$ as described above. The remarkable effectiveness of delta doping can be better understood by considering the doping-induced energy level shifts in organic materials. As excess free holes are generated in TCTA from $\mathrm{MoO}_{3}$ doping, the Fermi level shifts downward toward its HOMO edge due to an increased radical cation density. This effect has been confirmed by ultraviolet electron spectroscopy and Kevin probe [188-189]. In organic hole transport materials doped with a transition metal oxide, large downshifts of the Fermi level have been measured, in the range of $0.7-1 \mathrm{eV}$. At the undoped/doped interface, the Fermi level shift would cause energy level bending, leading to the formation of an interfacial potential barrier. We have found that an additional bias of $1.4 \mathrm{~V}$ must be applied to a simple hole-only device to overcome such a junction barrier between undoped $\mathrm{CBP}$ and $\mathrm{WO}_{3}$-doped $\mathrm{CBP}$. These doping effects may cause the energy band diagram to have a periodic potential well in a delta-doped layer sandwiched between two undoped spacer layers. Therefore, the periodically delta-doped TCTA containing multiple potential wells, which function as reservoirs for free holes. Such a unique nanostructure allows accumulation of a large number of holes in the doped regions. Furthermore, the holes are spatially separated from free electrons, and thus have an extended lifetime. All these effects may give rise to an average hole concentration higher than that in a uniformly doped organic film.

Under an applied electric field in the direction perpendicular to the film plane, holes move through the HTL via drifting or tunneling. With thick undoped spacer layers, tunneling is not possible, so holes must escape from the potential wells via thermionic emission and drift through the spacer layers. The overall hole transport capability is greater than that in an undoped HTL, but worse than that in a HTL with uniform doping. This is the case for the device with 10 $\mathrm{nm}$ spacer layers seen in Figure 5.14(b). When the spacer thickness is reduced to $3 \mathrm{~nm}$, holes may tunnel through the spacer layers, leading to more facile charge transport than in a uniformly 
doped HTL. In this case, the delta-doped structure acts as a doping superlattice with multiple coupled potential wells. On the other hand, when an electric field is applied in the direction parallel to the film plane, high-density localized holes drift fast along the potential wells and are not hampered by any energy barriers.

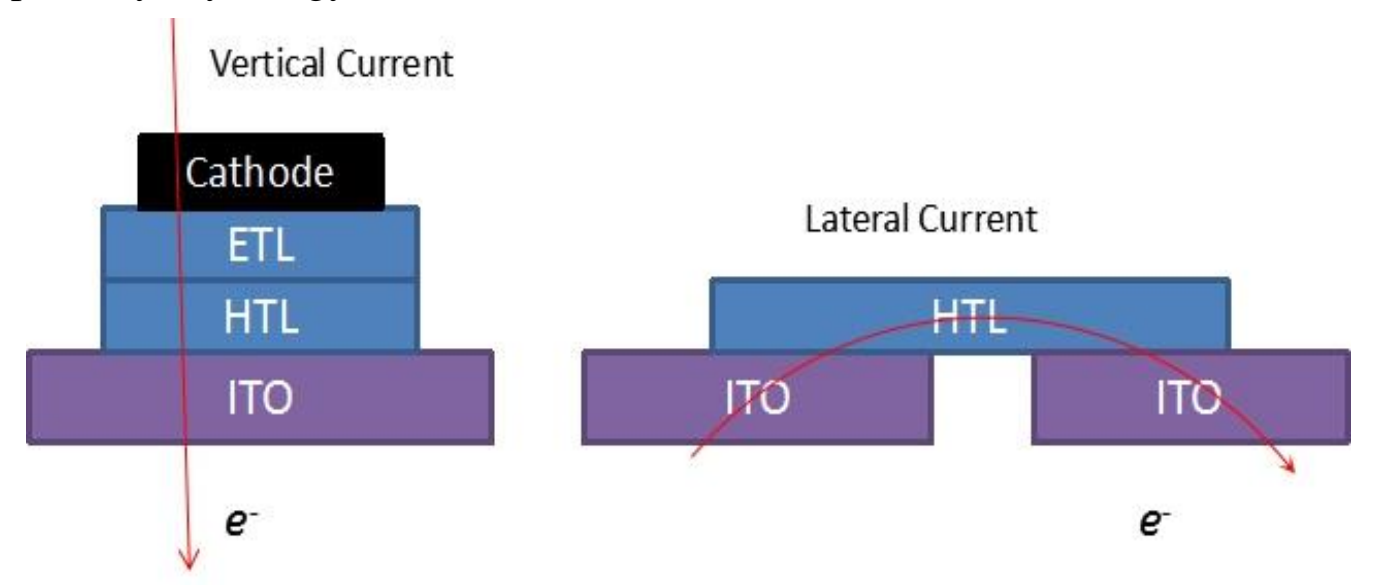

Figure 5.15 Schematic diagram of vertical and lateral current flows in delta-doped structures.

The periodic delta doping strategy may have a more important technological implication for organic devices which rely on lateral charge transport along the film plane but suffer from low charge mobility. Especially, the organic field-effect transistor can benefit from the concept of delta doping, which offers a promising approach for achieving quasi two-dimensional charge gas and a high- transconductance channel in organic materials. To examine lateral charge transport along the film plane in the delta-doped structure, we measured the current flowing laterally through the $\mathrm{MoO}_{3}$-doped TCTA HTL between the ITO anodes of two adjacent devices on the same substrate as shown in Figure 5.15.

As seen in Figure 5.16, the current in the undoped HTL was immeasurable $(<\mathrm{pA})$, whereas the current conduction in the delta-doped HTLs was greatly improved and showed a clear dependence on the spacer thickness. As the spacer layer thickness is reduced from $10 \mathrm{~nm}$ to $3 \mathrm{~nm}$, the current at $5 \mathrm{~V}$ increases from $8 \times 10^{-10} \mathrm{~A}$ to $8 \times 10^{-9} \mathrm{~A}$. As a comparison, the current in the structure uniformly doped with $30 \mathrm{~mol} \% \mathrm{MoO}_{3}$ is about $2 \times 10^{-10} \mathrm{~A}$ at $5 \mathrm{~V}$. These results bode well for the development of organic devices relying on a lateral charge transport plane such as 
the organic field-effect transistor which generally suffer from the low charge mobility of inorganic materials. The concept of delta doping may offer a promising approach for constructing high-transconductance organic channels.

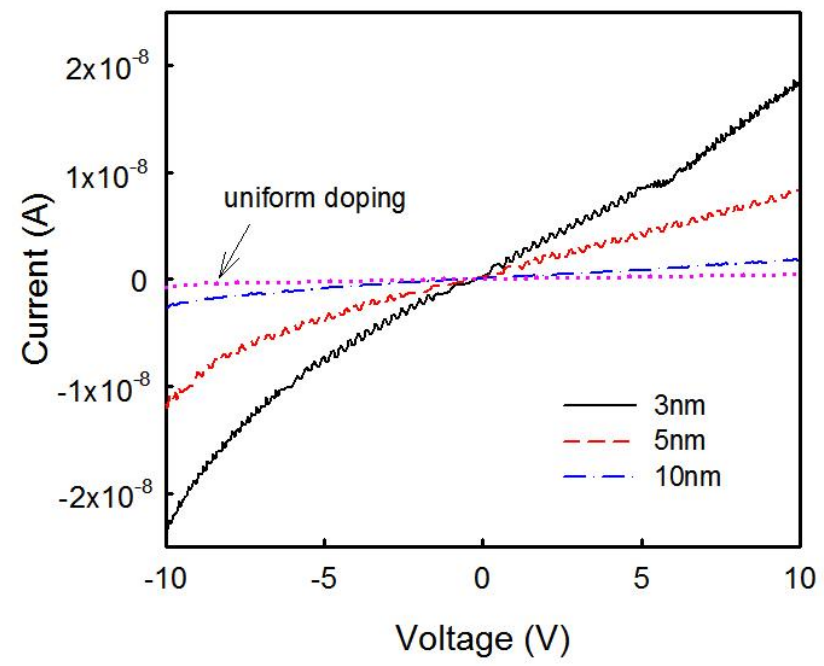

Figure 5.16 Lateral J-V characteristic of hole-only devices with uniform doped TCTA and delta-doped TCTA with 3-10 nm spacer as HTL

\subsection{Conclusions}

In summary, we developed selective doping of organic materials through simple sequential deposition of undoped organic spacer and TMO layers, and studied the effects of selective doping of wide bandgap hole transport materials including CBP and TCTA with TMOs. The effect of doping was a function of the undoped spacer layer thickness, and became saturated as the spacer was reduced to 3-5 nm. Hole-only devices with a HTL comprising alternative 0.5 nm TMO-doped/3 nm undoped layers exhibited comparable or even more efficient hole transport compared to devices with a uniformly doped HTL, and had a turn-on voltage as low as $1.1 \mathrm{~V}$. SIMS measurements showed that fast diffusion of evaporated metal oxides with high kinetic energy into CBP, which has a relatively low glass transition temperature. As a result, a nearly uniform doping profile was obtained. However, in TCTA which has a much higher glass transition temperature, the diffusion was slow, resulting in delta doping. A marked increase in the 
lateral conductively was observed in the delta-doped structure. The great doping effectiveness is explained by a high concentration of free holes retained in the delta-doped structure where periodic delta doping formed and created multiple potential wells acting as hole reservoirs. Simple Alq3-based green OLEDs with a selectively-doped CBP HTL showed a reduced voltage and markedly improved lifetime under constant-current stressing compared to similar OLEDs with an undoped HTL. These findings validate that selective doping is an alternative viable doping strategy for organic materials when co-deposition is not allowed or difficult to control and bode well for the development of high-transconductance organic transport devices. 


\section{Chapter 6 Conclusions}

\subsection{Conclusions}

This dissertation has focused on studying the effect of self-generated joule heating on degradation of OLEDs and improving the OLEDs performance especially the reliability by uniform or selective doping in the hole transport layer with transition metal oxides. Firstly, pulsed current stressing was applied to differentiate thermal and non-thermal factors causing the aging of OLEDs in an effort to identify the key factors affecting the device reliability. Secondly, inorganic doping effects of $\mathrm{WO}_{3}$ in $\mathrm{CBP}$ were fully investigated by fabricating hole-only devices and phosphorescent OLED (PhOLED) devices. The doping was optimized by adjusting doping concentration and doping layer thickness. High performance PhOLEDs having a simplified structure were obtained with $\mathrm{WO}_{3}$ doping in the CBP hole transport layer, and exhibited more reliable operation under high current density stressing. Finally, selective doping was introduced into organic hole transport materials by sequential deposition of organic materials and TMOs. The dopant diffusion was investigated by high-resolution SIMS measurements. The doping effect was proved comparable or even better than uniform doping. Delta doping formed in the selective doping of TCTA with $\mathrm{MoO}_{3}$ also improved the lateral current conduction, and thus may be utilized to develop high-transconductance organic transport devices.

The primary results of the research are summarized as follows:

1. Reliability Study of OLEDs (Chapter 3)

i. A simple yet effective method to investigate the joule heating effect on degradation of OLEDs was developed. By applying pulsed currents to stress OLEDs, the thermal and non-thermal factors were separated. The stressed devices exhibited increased low-bias leakage and series resistance. The luminance evolution consisted of an initial rapid decay regime and a subsequent slow decay regime, which were 
governed by different degradation mechanisms. A Prolonged lifetime was achieved by adding a reverse current which can remove the defects and alleviate charge accumulation.

ii. In PhOLEDs with a CBP HTL which had a relatively high interfacial energy barrier, stressing under $10 \%$ duty cycle pulsed current, a $70 \%$ increase in the effective half life was achieved compared to similar devices under CW current stressing. In contrast, in PhOLEDs with an NPB HTL where a relatively low interfacial energy barrier existed, only $\sim 15 \%$ improvement was obtained, indicating a minor heating effect. These results suggest that the PhOLED lifetime may be improved by reducing the interfacial barriers for charge injection and the total power dissipation during device operation.

2. P-type Doping of hole transport materials with Transition Metal Oxides (TMOs) in PhOLEDs (chapter 4)

i. The effects of $\mathrm{MoO}_{3}$ and $\mathrm{WO}_{3}$ doping in $\mathrm{CBP}$, a wide bandgap ambipolar organic material, were fully investigated through fabrication and comparative characterization of hole-only devices based on doped CBP. By varying the doping level from 10-40 mol\% and the doping thickness from 5-40 nm, we have found that, to achieve effective $\mathrm{WO}_{3}$ doping for improved hole injection and transport, the doped layer should be thicker than $10 \mathrm{~nm}$, and the doping level should be greater than $20 \mathrm{~mol} \%$. It was also found that an energy barrier exists at the doped/undoped CBP interface, resulting in an additional $\sim 1.4 \mathrm{~V}$ voltage drop in devices at 100 $\mathrm{mA} / \mathrm{cm}^{2}$. This was explained by doping-induced Fermi level shift toward the $\mathrm{HOMO}$ in the organic host. For $\mathrm{MoO}_{3}$ doping, similar results were obtained, but the doping effect saturated at a lower doping level $\sim 10 \mathrm{~mol} \%$.

ii. Simplified green PhOLEDs with CBP used as both the hole transport material and 
EML host were fabricated. With an $30 \% \mathrm{WO}_{3}$ doped HTL, brightness of 11163 $\mathrm{cd} / \mathrm{m}^{2}$ was achieved at $20 \mathrm{~mA} / \mathrm{cm}^{2}$. The corresponding EQE and current efficiency were $13.6 \%$ and $55.8 \mathrm{~cd} / \mathrm{A}$, respectively. The simplified PhOLEDs also exhibited a markedly improved lifetime under constant-current stressing compared to conventional undoped PhOLEDs, and thus represent a viable design for efficient and durable OLEDs suitable for display and lighting applications.

iii. The doping effect of TMO has been attributed to the formation of charge transfer complexes through electrons transferring from the HOMO of the organic host to the conduction band of the inorganic dopant. To validate this, doping of $\mathrm{CBP}$ with $\mathrm{ZnO}$ $\& \mathrm{SnO}_{2}$ whose conduction bands are higher than the $\mathrm{HOMO}$ of $\mathrm{CBP}$, was also investigated. The voltage of hole-only devices doped with $\mathrm{ZnO}$ or $\mathrm{SnO}_{2}$ sharply increased by more than $5 \mathrm{~V}$ at $20 \mathrm{~mA} / \mathrm{cm}^{2}$. This is expected as electrons from the conduction band of the n-type dopants may transfer to the organic host and reduce the net hole concentration in the host.

3. Selective Doping of organic hole transport materials with TMOs (Chapter 5)

i. Selective doping, is introduced as a new doping method, which can be done easily by sequential deposition instead of co-deposition. We studied the effects of selective doping of CBP and TCTA with $\mathrm{WO}_{3}$ and $\mathrm{MoO}_{3}$. The effect of selective doping was a strong function of the undoped spacer layer thickness, and became saturated as the spacer was reduced to 3-5 nm. Hole-only devices with a HTL comprising alternative $0.5 \mathrm{~nm}$ TMO-doped/3 nm undoped layers exhibited comparable or even more efficient hole transport compared to devices with a uniformly doped HTL, and had a turn-on voltage as low as $1.1 \mathrm{~V}$.

ii. SIMS measurements were taken to study the diffusion behaviors of the TMO dopant in the organic host. In CBP which has a low glass transition temperature of 
$52{ }^{\circ} \mathrm{C}$, the oxides underwent a fast transient diffusion driven by the high kinetic and thermal energies carried by the evaporated species. As a result, a nearly uniform doping profile with small concentration variations was obtained. For TCTA with a high glass transition temperature of $151^{\circ} \mathrm{C}$, much shorter diffusion lengths of a few nanometers were determined, resulting in good delta doping profiles with large concentration fluctuations. The delta doping effect was then explained by free hole accumulation in the potential wells formed in the narrow doped regions.

iii. Simple $\mathrm{Alq}_{3}$-based green OLEDs with a selectively doped CBP HTL showed a reduced voltage and a markedly improved lifetime under constant-current stressing compared to similar OLEDs with an undoped CBP HTL. The improvement was attributed to reduced power dissipation and localized heating in the selectively doped HTL. These findings validate that selective doping is an alternative viable doping strategy for organic materials and especially bode well for the development of high-transconductance organic transport devices.

The future work will center on the following two topics: (i) developing effective n-type doping in organic electron transport materials so simple, efficient, and reliable p-i-n OLEDs can be made; (ii) Employing delta doping to create high-transconductance organic channels for highperformance organic field-effect transistors. 


\section{References}

[1] Bergh, A., et al., "The Promise and Challenge of Solid state Lighting.", Physics Today, 2001. 54(12): p. 42-47.

[2] J.Y. Tsao, "Solid-state lighting: lamps, chips, and materials for tomorrow", IEEE Circuits and Devices Magazine, 2004. 20(3): p. 28-37

[3] K.T. Kamtekar, A. P. Monkman, M. R. Bryce, "Recent Advances in White Organic Light Emitting Materials and Devices (WOLEDs)", Adv. Mater, 2009. 22(5): p. 572-582

[4] C.M. Bourget, " An introduction to light-emitting diodes ", HortScience, 2008. 43(7): p.19441946

[5] S. Nakamura, " Current status of GaN-based solid-state lighting ", MRS Bulletin, 2009. 34(2): p. 101-107

[6] M. Kavehrad, " Sustainable energy-efficient wireless applications using light", IEEE

Communications Magazine, 2010. 48(12): p. 66-73

[7] J. Edmond, "Reinventing Lighting," DOE SSL R\&D Workshop, San Francisco, CA, 27 January 2015. [Online]. Available:

http://www.energy.gov/sites/prod/files/2015/02/f19/edmond_reinventing_sanfrancisco2015.p df. [8] E. lsbergen, "OLEDs for lighting applications.” Proceedings of SPIE, 2008. 7051(1): p. $70511 \mathrm{~A}$.

[9] E. Mainea, E. Garnsey, " Commercializing generic technology: The case of advanced materials ventures," Research Policy, 2006. 35(3): p. 375-393

[10] C. H. Oh, H. J. Shin, W. J. Nam, B. C. Ahn, S. Y. Cha, S. D. Yeo, "Technological Progress and Commercialization of OLED TV", 2013. 44(1): p. 239-242

[11] J. Brown, R. Kwong, Y. J. Tung, V. Adamovich, M. Weaver and M. Hack, "Recent progress in high-efficiency phosphorescent OLED technology", 2004. 12(3): p. 329-332

[12] M. Pope, H. P Kallmann, and P. Magnante, J.Chem. Phys. 38, 2042 (1963); M.Sano, M.Pope, and H. Kallman, J. Chem. Phys. 43, 2920 (1965)

[13] W. Helfrich and W. G. Schneider, Phys. Rev. Lett. 14, 229 (1965); W. Helfrich and W.G.

Schneider, J. Chem. Phys. 44, 2902 (1965)vJohnson, J., The End of The Light Blub. Chemical \& Engineering News, 2007. 85(49): p.46-51.

[14] P. S. Vincentt, W. A. Barlow, R. A. Hann, and G. G. Roberts, Thin Solid Films 94, 171 (1982)

[15] D. Braun, A. J. Heeger. "Visible light emission from semiconducting polymer diodes." Appl.

Phys. Lett. 1991. 58: p.1982-198

[16] C. W. Tang and S. A. VanSlyke, "Organic electroluminescent diodes" Appl. Phys. Lett. 1987. 
51: 913

[17] C. Adachi, S. Tokito, T. Tsutsui and S. Saito, "Electroluminescence in organic films with threelayer structure", Jpn. J. Appl. Phys. 27 L269 (1988)

[18] J. H. Burroughes, D. D. C. Bradley, A. R. Brown, R. N. Marks, K. MacKay, R. H. Friend, P.

L. Burns and A. B. Holmes, " Light-emitting diodes based on conjugated polymers," Nature. 347, 539 (1990).

[19] J. Kido, M. Kimura and K. Nagai, " Multilayer white light-emitting organic electroluminescent device" Science. 26719951332.

[20] M. A. Baldo, D. F. O'Brien, Y. You, A. Shoustikov, S. Sibley, M. E. Thompson and S. R. Forrest, " Highly efficient phosphorescent emission from organic electroluminescent devices," Nature 395, 151 (1998)

[21] DOE SSL Multi-year program plan, 2012

[22] DOE SSL Multi-year program plan, 2013

[23] Forrest, S.R., "The path to ubiquitous and low-cost organic electronic appliances on plastic." Nature, 2004. 428(6986): p. 911-918.

[24] So, F., J. Kido, and P. Burrows, "Organic Light-Emitting Devices for Solid-State Lighting." MRS Bulletin, 2008. 33: p. 663-660.

[25] V. Elsbergen, et al., "OLEDs for lighting applications." Proceedings of SPIE, 2008. 7051(1): p. $70511 \mathrm{~A}$.

[26] Hack, M., OLED Lighting - Completing the SSL Portfolio. DOE SSL Manufacturing Workshop, 2009.

[27] P. A. Lane, J. Shinar and K. Yoshino, Phys. Rev. B 54, 9308 (1996).

[28] Handbook of Organic Conductive Molecules and Polymers: Vol. 3. Conductive Polymer:

Spectroscopy and Physical Properties, edited by H. S. Nalwa (J. Wiley, New York, 1997).

[29] E. J. W. List, C. H. Kim, J. Shinar, A. Pogantsch, G. Leising and W. Graupner, Appl. Phys.

Lett. 76, 2083 (2000). 
[30] E. J. W. List, J. Partee, J. Shinar, U. Scherf, K. Müllen, E. Zojer, K. Petritsch, G. Leising and W. Graupner, Phys. Rev. B 61, 10807 (2000).

[31] M.G. Helander, Z.B. Wang, etl. "Chlorinated indium tin oxide electrodes with high work function for organic device compatibility," Science 332, 944 (2011).

[32] R. Meerheim, S. Scholz, S. Olthof, G. Schwartz, et. al, "Influence of charge balance and exciton distribution on efficiency and lifetime of phosphorescent organic light-emitting devices." J. Appl. Phys. 2008, 104, 014510.

[33] Scholz, S.; Lüssem, B.; Leo, K. Chemical changes on the green emitter tris(8-hydroxyquinolinato)aluminum during device aging of pi- n-structured organic light emitting diodes. Appl. Phys. Lett. 2009, 95,183309.

[34] Soltzberg, L. J.; Slinker, J. D.; Flores-Torres, S.; Bernards, D. A.; Malliaras, G. G.; Abruña, H. D.; Kim, J.-S.; Friend, R. H.; Kaplan, M. D.; Goldberg, V. Identification of a Quenching Species in Ruthenium Tris-Bipyridine Electroluminescent Devices. J. Am. Chem. Soc. 2006,128, $7761-7764$.

[35] Sivasubramaniam, V.; Brodkorb, F.; Hanning, S.; Loebl, H. P.; van Elsbergen, V.; Boerner, H.; Scherf, U.; Kreyenschmidt, M. Investigation of FIrpic in PhOLEDs via LC/MS technique. Cent. Eur. J. Chem. 2009, 7,836-845.

[36] Scholz, S.; Meerheim, R.; Lüssem, B.; Leo, K. Laser desorption/ionization time-of-flight mass spectrometry: A predictive tool for the lifetime of organic light emitting devices. Appl. Phys. Lett. 2009, 94,043314.

[37] Van Slyke, S. A.; Chen, C. H.; Tang, C. W. Organic electroluminescent devices with improved stability. Appl. Phys. Lett. 1996, 69, 2160-2162.

[38] Burrows, P. E.; Shen, Z.; Bulovic, V.; McCarty, D. M.; Forrest, S. R.; Cronin, J. A.; Thompson, M. E. Relationship between electroluminescence and current transport in organic heterojunction lightemitting devices. J. Appl. Phys. 1996, 79, 7991-8006. 
[39] Féry, C.; Racine, B.; Vaufrey, D.; Doyeux, H.; Cinà, S. Physical mechanism responsible for the stretched exponential decay behavior of aging organic light-emitting diodes. Appl. Phys. Lett. $2005,87,213502$.

[40] Jarikov, V. V.; Kondakov, D. Y.; Brown, C. T. Efficient and extremely long-lived organic light-emitting diodes based on dinaphthylperylene. J. Appl. Phys. 2007, 102, 104908.

[41] Howard, W. E.; Prache, O. F. Microdisplays based upon organic light-emitting diodes. IBM J. Res. Dev. 2001, 45, 115-127.

[42] Giebink, N. C.; D’ Andrade, B. W.; Weaver, M. S.; Mackenzie, P. B.; Brown, J. J.; Thompson, M. E.; Forrest, S. R. Intrinsic luminance loss in phosphorescent small-molecule organic light emitting devices due to bimolecular annihilation reactions. J. Appl. Phys. 2008, 103, 044509.

[43] Pfeiffer, M.; Forrest, S. R. Organic Light Emitting Devices. In Nanoelectronics and Information Technology - Advanced Electronic Materials and Devices, 2nd ed.; Waser, T., Ed.; Wiley-VCH: Weinheim, 2005; pp 915-932.

[44] Chwang, A. B.; Kwong, R. C.; Brown, J. J. Graded mixed-layer organic light-emitting devices. Appl. Phys. Lett. 2002, 80, 725-727.

[45] D.A. Clymer, M.A. Matin," Characterization of thin - metal anode buffers in organic devices ", Microwave and Optical Technology Letters, 2006. 48(10): p.2070-2072 [46] http://www.bmbf.de/foerderungen/4389.php (accessed Aug 15, 2013).

[47] Birnstock, J.; Canzler, T.; Hofmann, M.; Lux, A.; Murano, S.; Wellmann, P.; Werner, A. PIN OLEDs Improved Structures and Materials to Enhance Device Lifetime and Ease Mass Production. Dig. Tech. Pap. - Soc. Inf. Disp. Int. Symp. 2007, 38, 1193-1196. [48] Birnstock, J.; Canzler, T.; Hofmann, M.; Lux, A.; Murano, S.; Wellmann, P.; Werner, A. PIN OLEDs Improved structures and materials to enhance device lifetime. J. Soc. Inf. Disp. 2008, 16, 
$221-229$.

[49] A.H. Wilson, The Electrical Conductivity of the Transition Metals. Proceedings of the Royal Society of London. Series A. Mathematical and Physical Sciences, 1938. 167(931): p. 580-593.

[50] W. Brütting, Physics of Organic Semiconductors. 2005, Berlin: Wiley-VCH.

[51] C. K. Chiang, C. R. Fincher, Y. W. Park, A. J. Heeger, H. Shirakawa, E. J. Louis, S. C. Gau, and A. G. MacDiarmid, Electrical Conductivity in Doped Polyacetylene, Phys. Rev. Lett. 39, 1098-1101 (1977).

[52] A. Gadisa, Studies of charge transport and energy levels in solar cells based on polymer/fullerene bulk heterojunction; Linkoping Studies in Science and Technology Dissertation No. 1056

[53] The spin orbital interaction is proportional to $\mathrm{Z8}$, where $\mathrm{Z}$ is that atomic number of the atom [54] J. H. Burroughes, D. D. C. Bradley, A. R. Brown, R. N. Marks, K. MacKay, R. H.

Friend, P. L. Burns and A. B. Holmes, Nature 347, 539 (1990).

[55] S. A. Choulis, V. E. Choong, M. K. Mathai and F. So, Appl. Phys. Lett. 87, 113503 (2005).

[56] Z. B. Wang, M. G. Helander, J. Qiu, D. P. Puzzo, M. T. Greiner, Z. W. Liu and Z.

H. Lu, Appl. Phys. Lett. 98, 073310 (2011).

[57] Z. T. Xie, W. H. Zhang, B. F. Ding, X. D. Gao, Y. T. You, Z. Y. Sun, X. M.

Ding and X. Y. Hou, Appl. Phys. Lett. 94, 063302 (2009).

[58] P. Piromreun, H. Oh, Y. Shen, G. G. Malliaras, J. C. Scott and P. J. Brock, Appl. Phys. Lett. 77, 2403 (2000).

[59] T. Matsushima, G.-H. Jin, and H. Murata, J. Appl. Phys. 104, 054501 (2008).

[60] Y. Zheng, S-H. Eom, N. Chopra, J. Lee, F. So, and J. Xue, Appl. Phys. Lett. 92, 223301 (2008).

[61] G. Xie, Y. Meng, F. Wu, C. Tao, D. Zhang, M. Liu, Q. Xue, W. Chen and Y. Zhao, Appl. Phys. Lett. 92, 093305 (2008).

[62] S.-H. Eom, Y. Zheng, N. Chopra, J. Lee, F. So and J. Xue, Appl. Phys. Lett. 93, 
133309 (2009).

[63] V. Bulovic, G. Gu, P. E. Burrows, M. E. Thompson and S. R. Forrest, Nature 380, 29 (1996).

[64] G. Parthasarathy, P. E. Burrows, V. Khalfin, V. G. Kozlov and S. R. Forrest, Appl. Phys. Lett. 72, 2138 (1998).

[65] M. E. Thompson, S. R. Forrest and P. Burrows, US patent (1999).

[66] Novaled. Retrieved on 2011-10-04.

[67] T.-Y. Chu, J.-F. Chen, S.-Y. Chen, C.-J. Chen and C. H. Chen, Appl. Phys. Lett. 89, 053503 (2006).

[68] T. W. Lee, T. Noh, B. K. Choi, M. S. Kim, D. W. Shin and J. Kido , Appl. Phys. Lett. 92, 043301 (2008).

[69] J. Kido, Dig. Tech. Pap.-Soc. Inf. Disp. Int. Symp. 39, 931 (2008).

[70] J. C. Scott and G. G. Malliaras, Chem. Phys. Lett. 299, 115 (1999).

[71] R. H. Fowler and L. Nordheim, Proc. Roy. Soc. 121, 626 (1928).

[72] I. D. Parker, J. Appl. Phys. 75, 1658 (1995).

[73] Z. Chiguvare, J. Parisi and V. Dyakonov, J. Appl. Phys. 94, 2440 (2003).

[74] M. A. Abkowitz, H. A. Mizes and J. S. Facci, Appl. Phys. Lett. 66, 1288 (1995).

[75] E. M. Conwell and M. W. Wu, Appl. Phys. Lett. 70, 1867 (1997).

[76] V. I. Arkhipov, E. V. Emelianova, Y. H. Tak and H. Bässler, J. Appl. Phys. 84, 848 (1998).

[77] U. Wolf, V. I. Arkhipov and H. Bässler, Phys. Rev. B 59, 7507 (1999).

[78] S. Narioka, H. Ishii, D. Yoshimura, M. Sei, Y. Ouchi, K. Seki, S. Hasegawa, T. Miyazaki, Y. Harima and K. Yamashita, Appl. Phys. Lett. 67, 1899 (1995).

[79] H. Ishii, K. Sugiyama, E. Ito and K. Seki, Adv. Mater. 11, 605 (1999).

[80] T. Shimada, K. Hamaguchi, A. Koma and F.S. Ohuchi, Appl. Phys. Lett. 72, 1869 (1998). 
[81] R. Schlaf, P. G. Schroeder, M. W. Nelson, B. A. Parkinson, P. A. Lee, K. W.

Nebesny and N. R. Armstrong, J. Appl. Phys. 86, 1499 (1999).

[82] J. M. Barathan and Y. Yang, J. Appl. Phys. 84, 3207 (1998).

[83] J. Kalinowski, Mol. Cryst. Liq. Cryst. 355, 231 (2001).

[84] S.R. Forrest, D.D.C. Bradley, M.E. Thomson, Adv. Mater. 15 (2003) 1043.

[85] N.K. Patel, S. Cinà, J.H. Burroughes, IEEE J. Select. Top. Quant. Electron. 8 (2002) 346 .

[86] V. Cleave, G. Yahioglu, P. Le Barney, R. Friend, N. Tessler, Adv. Mater. 20 (1999) 285 .

[87] D.S. Mehta, K. Saxena, in: Proceedings of the Ninth Asian Symposium on Information Display (ASID-06), 2006, p. 198.

[88] S.R. Forrest, Org. Electron. 4 (2006) 45.

[89] G. Gu, D.Z. Garbuzov, P.E. Burrows, S. Venkatesh, S.R. Forrest, M.E. Thompson, Opt. Lett. 22 (1997) 396.

[90] J.-S. Kim, P.K.H. Ho, N.C. Greenham, R.H. Friend, J. Appl. Phys. 88 (2000) 1073.

[91] C.F. Madigan, M.H. Lu, J.C. Strurn, Appl. Phys. Lett. 76 (2000) 1650.

[92] W. Li, R.A. Jones, S.C. Allen, J.C. Heikenfield, J. Disp. Tech. 2 (2006) 143.

[93] M. Tammer, R.W. Higgins, A.P. Monkman, J. Appl. Phys. 91 (2002) 4010.

[94] M.H. Lu, C.F. Madigan, J.C. Strum, IEDM 00-07 (2000) 607.

[95] T. Nakamura, N. Tsutsumi, N. Juni, H. Fujii, J. Appl. Phys. 97 (2005) 054505.

[96] T. Yamasaki, K. Sumioka, T. Tsutsui, Appl. Phys. Lett. 76 (2000) 1243.

[97] K. Neyts, A.U. Nieto, J. Opt. Soc. Am. A 23 (2006) 1201.

[98] J.J. Shiang, T.J. Faircloth, A.R. Duggal, J. Appl. Phys. 95 (2004) 2889.

[99] R. Bathelt, D. Buchhauser, C. Gärditz, R. Paetzold, P. Wellmann, Org. Electron. 8 (2007) 293.

[100] S. Moller, S.R. Forrest, J. Appl. Phys. 91 (2002) 3324. 
[101] H.J. Peng, Y.L. Ho, C.F. Qiu, M. Wong, H.S. Kwok, SID 04 Digest (2004) 158.

[102] M.-K. Wei, I.-L. Su, Opt. Exp. 12 (2004) 5777.

[103] H. Peng, Y.L. Ho, X.-J. Yu, M. Wong, H.-S. Kwok, J. Disp. Tech. 1 (2005) 278.

[104] M.-K. Wei, I.-L. Su, Y.-J. Chen, M. Chang, H.-Y. Lin, T.-C. Wu, J. Micromech.

Microeng. 16 (2006) 368.

[105] J. Lim, S.S. Oh, D.Y. Kim, S.H. Cho, I.T. Kim, S.H. Han, H. Takezoe, E.H. Choi, G.S.

Cho, Y.H. Seo, S.O. Kang, B. Park, Opt. Exp. 14 (2006) 6564.

[106] V. Bulovic, V.B. Khalfin, G. Gu, P.E. Burrows, D.Z. Garbuzov, S.R. Forrest, Phys.

Rev. B 58 (1998) 3730.

[107] J. Lee, N. Chopra, F. So, Appl. Phys. Lett. 92 (2008) 033303.

[108] E.F. Schubert, N.E.J. Hunt, M. Micovic, R.J. Malik, D.L. Sivco, A.Y. Cho, G.J. Zydzik, Science 265 (1994) 943.

[109] T. Shiga, H. Fujikawa, Y. Taga, J. Appl. Phys. 93 (2003) 19.

[110] X. Liu, D. Poitras, Y. Tao, C. Py, J. Vac. Sci. Technol. 22 (2004) 764.

[111] C.-L. Lin, H.-W. Lin, C.-C. Wu, Appl. Phys. Lett. 87 (2005) 021101.

[112] J.R. Tischler, M.S. Bradley, V. Bulovic, J.H. Song, A. Numikko, Phys. Rev. Lett. 95 (2005)

036401.

[113] H. Peng, J. Sun, X. Zhu, X. Yu, W. Wong, H.-S. Kwok, Appl. Phys. Lett. 88 (2006) 073517.

[114] T.-Y. Cho, C.-L. Lin, C.-C. Wu, Appl. Phys. Lett. 88 (2006) 111106.

[115] C.-J. Yang, S.-H. Liu, H.-H. Hsieh, C.-C. Liu, T.-Y. Cho, C.-C. Wu, Appl. Phys. Lett. 91 (2007) 253508.

[116] L.H. Smith, J.A.E. Wasey, W.L. Barnes, Appl. Phys. Lett. 84 (2004) 2986.

[117] H. Kanno, Y. Sun, S.R. Forrest, Appl. Phys. Lett. 86 (2005) 263502.

[118] F.J. Duarte, L.S. Liao, K.M. Vaeth, Opt. Lett. 30 (2005) 3072.

[119] H. Kanno, N.C. Giebink, Y. Sun, S.R. Forrest, Appl. Phys. Lett. 89 (2006) 023503.

[120] L.S. Liao, K.P. Klubek, C.W. Tang, Appl. Phys. Lett. 84 (2004) 167. 
[121] H. Peng, J. Sun, X. Zhu, X. Yu, M. Wong, H.-S. Kwoka, Appl. Phys. Lett. 88 (2006) 073517 .

[122] F. Guo, D. Ma, Appl. Phys. Lett. 87 (2005) 173510.

[123] J. Shen, D. Wang, E. Langlois, W.A. Barrow, P.J. Green, C.W. Tang, J. Shi, Synth. Met. $111-112(2000) 233$.

[124] D.Y. Kondakov, W.C. Lenhart, W.F. Nichols, J. Appl. Phys. 101 (2007)

024512 .

[125] N.C. Giebink, B.W. D’Andrade, M.S. Weaver, J.J. Brown, S.R. Forrest, J.Appl. Phys. 105 (2009) 124514.

[126] A. Pinato, M. Meneghini, A. Cester, N. Wrachien, A. Tazzoli, E. Zanoni, G. Meneghesso, B. D’Andrade, J. Esler, S. Xia, J. Brown, in: Proc. IEEE 47th Annual Int. Reliability Phys. Symp., 2009, p. 253.

[127] X.A. Cao, Y.Q. Zhang, Appl. Phys. Lett. 100 (2012) 183304.

[128] H. Meng, N. Herron, in: Z. Li, H. Meng (Eds.), Organic Light-Emitting Materials and Devices, Taylor \& Francis, 2007, p. 295.

[129] H. Aziz, Z.D. Popovic, Chem. Mater. 16 (2004) 4522.

[130] S.C. Xia, R.C. Kwong, V.I. Adamovich, M.S. Weaver, J.J. Brown, in: Proc. IEEE 45th Annual Int. Reliability Phys. Symp., 2007, p. 253.

[131] H. Yamamoto, C. Adachi, M.S. Weaver, J.J. Brown, Appl. Phys. Lett. 100 (2012) 183306.

[132] M.S. Weaver, R.C. Kwong, V.A. Adamovich, M. Hack, J.J. Brown, J. Soc. Inf. Disp. 14 (2006) 449.

[133] H. Murata, C.D. Merritt, H. Inada, Y. Shirota, Z.H. Kafafi, Appl. Phys. Lett. 75 (1999) 3252 .

[134] J. Shen, D. Wang, E. Langlois, W.A. Barrow, P.J. Green, C.W. Tang, J. Shi, Synth. Met. $111-112$ (2000) 233.

[135] D.Y. Kondakov, W.C. Lenhart, W.F. Nichols, J. Appl. Phys. 101 (2007) 024512. 
[136] N.C. Giebink, B.W. D’Andrade, M.S. Weaver, J.J. Brown, S.R. Forrest, J. Appl. Phys. 105 (2009) 124514.

[137] A. Pinato, M. Meneghini, A. Cester, N. Wrachien, A. Tazzoli, E. Zanoni, G. Meneghesso, B. D’Andrade, J. Esler, S. Xia, J. Brown, in: Proc. IEEE 47th Annual Int. Reliability Phys. Symp., 2009, p. 253.

[138] X.A. Cao, Y.Q. Zhang, Appl. Phys. Lett. 100 (2012) 183304.

[139] D.G. Moon, R.B. Pode, C.J. Lee, J.I. Hars, Appl. Phys. Lett. 85 (2004) 4771.

[140] V.K. Chandra, B.P. Chandra, Org. Electron. 13 (2012) 329.

[141] H. Aziz, Z.D. Popovic, N.X. Hu, Appl. Phys. Lett. 81 (2002) 370.

[142] R. Acharya, X.A. Cao, Appl. Phys. Lett. 101 (2012) 053306.

[143] D. Zou, M. Yahiro, T. Tsutsui, Appl. Phys. Lett. 72 (1998) 2484.

[144] R. Meerheim, B. Lussem, K. Leo, Proc. IEEE 97 (2009) 1606.

[145] X. Zhou, J. Blochwitz, M. Pfeiffer, A. Nollau, T. Fritz, and K. Leo, Adv. Funct. Mater. 11 (2001) 310 .

[146] M. Kröger, S. Hamwi, J. Meyer, T. Riedl, W. Kowalsky, A. Kahn, Org. Electron. 10 (2009) 932.

[147] J. H. Lee, H. M. Kim, K. B. Kim, J. J. Kim, Org. Electron. 12 (2011) 950.

[148] T. Glaser, S. Beck, B. Lunkenheimer, D. Donhauser, A. Köhn, M. Kröger, A. Pucci, Org. Electron. 14 (2013) 575.

[149] X. Qiao, J. Chen, X. Li, and D. Ma, J. Appl. Phys. 107 (2010) 104505.

[150] W. J. Shin, J. Y. Lee, J. C. Kim, T. H. Yoon, T. S. Kim, O. K. Song, Org. Electron. 9 (2008) 333.

[151] S. Hamwi, J. Meyer, T. Winkler, T. Riedl, and W. Kowalsky, Appl. Phys. Lett. 94 (2009) 253307.

[152] C. Yun, G. Xie, C. Murawski, J. Lee, F. Ventsch, K. Leo, M. C. Gather, Org. Electron. 14 (2013) 1695. 
[153] F. Wang, X. Qiao, T. Xiong, and D. Ma, J. Appl. Phys. 105 (2009) 084518.

[154] J. Meyer, S. Hamwi, S. Schmale, T. Winkler, H. H. Johannes, T. Riedl and W. Kowalsky, J. Mater. Chem. (2009) 702.

[155] S. Y. Kim, W. S. Jeon, T. J. Park, R. Pode, J. Jang, and J. H. Kwon, Appl. Phys. Lett. 94 (2009) 133303.

[156] M. T. Hsieh, M. H. Ho, K. H. Lin, J. F. Chen, T. M. Chen, and C. H. Chen, Appl. Phys. Lett. 95 (2009) 033501.

[157] Y. Q. Zhang, R. Acharya, and X. A. Cao, J. Appl. Phys. 112 (2012) 013103.

[158] K. Harada, M. Riede, K. Leo, O.R. Hild, C.M. Elliott, Phys. Rev. B 77 (2008) 195212.

[159] J. Meyer, S. Hamwi, M. Kröger, W. Kowalsky, T. Riedl and A. Kahn, Adv. Mater. 24 (2012) 5408.

[160] R. Acharya and X. A. Cao, IEEE J. Display Technol. 9 (2013) 942.

[161] X. M. Li and X. A. Cao, Org. Electron. 14 (2013) 2523.

[162] Brütting, W., Physics of Organic Semiconductors. 2005, Berlin: Wiley-VCH.

[163] Farchioni, R. and G. Grosso, Organic Electronic Materials. 2001, Heidelberg: Springer.

[164] Chang, C.-C., et al., Highly power efficient organic light-emitting diodes with a p-doping layer. Applied Physics Letters, 2006. 89(25): p. 253504-3.

[165] Kröger, M., et al., Temperature-independent field-induced charge separation at doped organic/organic interfaces: Experimental modeling of electrical properties. Physical Review B, 2007. 75(23): p. 235321.

[166] Ho, M.-H., et al., A morphologically stable host material for efficient phosphorescent green and red organic light emitting devices. Thin Solid Films, 2008. 517(2): p. 943-947.

[167] Schubert, E. F. Delta doping of III-V compound semiconductors: fundamentals and device applications. J. Vac. Sci. Technol. A 8, 2980-2996 (1990)

[168] H. Sato, T. Minami, S. Takata, and T. Yamada, Thin Solid Films 236, 27 1993

[169] R. Meerheim, B. Lussem, K. Leo, Proc. IEEE 97 (2009) 1606.

[170] M. Pfeiffer, S. R. Forrest, K. Leo, M. E. Thompson, Adv. Mater. 14 (2002) 1633. 
[171] B. W. D'Andrade, S. R. Forrest, A. B. Chwang, Appl. Phys. Lett. 83 (2003) 3858.

[172] D. S. Leem, S. Y. Kim. J. J. Kim. M. H. Chen, C. I. Wu, Electrochem. Solid-State Lett. 12 (2009) J8.

[173] J. Meyer, S. Hamwi, M. Kröger, W. Kowalsky, T. Riedl, A. Kahn, Adv. Mater. 24 (2012) 5408.

[174] T. Glaser, S. Beck, B. Lunkenheimer, D. Donhauser, A. Köhn, M. Kröger, A. Pucci, Org. Electron. 14 (2013) 575.

[175] P. C. Kao, C. T. Chiu, Org. Electron. 26 (2015) 443.

[176] S. Hamwi, J. Meyer, T. Winkler, T. Riedl, W. Kowalsky, Appl. Phys. Lett. 94 (2009) 253307.

[177] F. Wang, X. Qiao, T. Xiong, D. Ma, J. Appl. Phys. 105 (2009) 084518.

[178] M. Kröger, S. Hamwi, J. Meyer, T. Riedl, W. Kowalsky, A. Kahn, Org. Electron. 10 (2009) 932.

[179] Y. Zhao, J. Zhang, S. Liu, Y. Gao, X. Yang, K. S. Leck, A. P. Abiyasa, Y. Divayana, E. Mutlugun, S. T. Tan, Q. Xiong, H. V. Demir, X. W. Sun, Org. Electron. 15 (2014) 871.

[180] J. Meyer, S. Hamwi, S. Schmale, T. Winkler, H. H. Johannes, T. Riedl, W. Kowalsky, J. Mater. Chem. 19 (2009) 702.

[181] X. Qiao, J. Chen, X. Li, D. Ma, J. Appl. Phys. 107 (2010) 104505.

[182] J. Qiu, Thesis, University of Toronto, 2012.

[183] X. M. Li, X. A. Cao, Organic Electron. 17 (2015) 9.

[184] X. A. Cao, Y. Q. Zhang, Appl. Phys. Lett. 100 (2012) 183304.

[185] D. Zou, M. Yahiro, T. Tsutsui, Appl. Phys. Lett. 72 (1998) 2484.

[186] R. Meerheim, B. Lussem, K. Leo, Proc. IEEE 97 (2009) 1606.

[187] X. Zhou, J. Blochwitz, M. Pfeiffer, A. Nollau, T. Fritz, and K. Leo, Adv. Funct. Mater. 11 (2001) 310 .

[188] M. Kröger, S. Hamwi, J. Meyer, T. Riedl, W. Kowalsky, A. Kahn, Org. Electron. 10 (2009) 932.

[189] J. H. Lee, H. M. Kim, K. B. Kim, J. J. Kim, Org. Electron. 12 (2011) 950. 